Supporting Information for

\title{
Palladium-Catalyzed Cascade Arene/Alkyne Annulation: Synthesis of Fluorene-Benzoxazine Derivatives
}

\author{
Zhong-Jian Cai, Fang-Hui Li, Shun-Yi Wang* and Shun-Jun Ji* \\ Key Laboratory of Organic Synthesis of Jiangsu Province, College of Chemistry, Chemical Engineering and \\ Materials Science \& Collaborative Innovation Center of Suzhou Nano Science and Technology, Soochow University, \\ Suzhou 215123, China \\ E-mail: shunyi@suda.edu.cn; shunjun@suda.edu.cn
}

\section{Table of Contents}

Experimental Section $-S 2$

Optimization of The Reaction Conditions S3

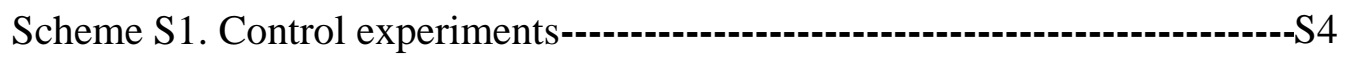

Characterization Data of Compounds $\mathbf{2 a - 2 t}$ and $\mathbf{2 a}$ ' - -S5-S1 1

Copies of ${ }^{1} \mathrm{H}$ and ${ }^{13} \mathrm{C}$ NMR Spectra for Compounds $\mathbf{2 a - 2 t}$ and $\mathbf{2 a}$ ' $\mathrm{S} 12-\mathrm{S} 32$

General procedure for synthesis of starting materias 1a-1t S33-S36

Characterization Data of starting materias 1a-1t S37-S42

Copies of ${ }^{1} \mathrm{H}$ and ${ }^{13} \mathrm{C}$ NMR Spectra for starting materias $\mathbf{1 a - 1 t}$ S43-S62

Kinetic isotope effect studies S63-S68 


\section{Experimental Section}

\section{General}

Melting points were recorded on an Electrothermal digital melting point apparatus and were uncorrected. IR spectra were recorded on a BRUKER VERTEX 70 spectrophotometer. ${ }^{1} \mathrm{H}$ NMR and ${ }^{13} \mathrm{C}$ NMR spectra were recorded on a BRUKER $400 \mathrm{MHz}\left({ }^{1} \mathrm{H} \mathrm{NMR}\right)$ and $100 \mathrm{MHz}\left({ }^{13} \mathrm{C}\right.$ NMR) spectrumeter using $\mathrm{CDCl}_{3}$ or DMSO- $d_{6}$ as solvent and TMS as internal standard. High resolution mass spectra were obtained using BRUKER micrOTOF-Q III instrument with ESI source.

\section{Typical procedure for the construction of 2a:}

The substrate $N$-(2-([1,1'-biphenyl]-2-ylethynyl)phenyl)benzamide (1a, $0.5 \mathrm{mmol}, 0.1866 \mathrm{~g})$, BQ (1.0 mmol, 0.1081g, 2.0 equiv.) and $\mathrm{Pd}(\mathrm{OAc})_{2}(0.025 \mathrm{mmol}, 0.0056 \mathrm{~g}, 5 \mathrm{~mol} \%)$ were added to a $25 \mathrm{~mL}$ Schlenk tube. The flask was evacuated and backfilled with $\mathrm{O}_{2}$, followed by addition of DMSO $(2.0 \mathrm{~mL})$. The mixture was stirred at $60{ }^{\circ} \mathrm{C}$ for $6 \mathrm{~h}$, then added $\mathrm{Pd}(\mathrm{OAc})_{2}(0.025 \mathrm{mmol})$ and stirred at $60{ }^{\circ} \mathrm{C}$ for another $6 \mathrm{~h}$. The solution was then quenched by $\mathrm{H}_{2} \mathrm{O}$ and extracted with EtOAc, the combined organic layers were dried over $\mathrm{Na}_{2} \mathrm{SO}_{4}$, filtered, and evaporated under vaccum. The residue was purified by column chromatography on silica gel (eluent: light petroleum ether : ethyl acetate, $V: V=20: 1)$ to afford the desired product 4-(9H-fluoren-9-ylidene)-2-phenyl-4H-benzo[d][1,3]oxazine $\mathbf{2 a}$. 


\section{Optimization of The Reaction Conditions:}

Table S1: The reaction with different oxidant. ${ }^{\mathrm{a}}$

\begin{tabular}{|c|c|c|c|c|}
\hline entry & $\begin{array}{c}\text { cat. } \\
\text { (equiv) }\end{array}$ & oxidant & solvent $(\mathbf{m L})$ & $\begin{array}{l}\text { Yield(\%) } \\
\text { (LC-MS) }\end{array}$ \\
\hline 1 & $\mathrm{Pd}(\mathrm{OAc})_{2}(0.1)$ & $\mathrm{BQ}(0.5$ equiv $)+\mathrm{O}_{2}$ & DMSO (2) & 44 \\
\hline 2 & $\mathrm{Pd}(\mathrm{OAc})_{2}(0.1)$ & $\mathrm{O}_{2}$ & $\operatorname{DMSO}(2)$ & 23 \\
\hline 3 & $\mathrm{Pd}(\mathrm{OAc})_{2}(0.1)$ & BQ ( 0.5 equiv $)+$ air & DMSO (2) & 36 \\
\hline 4 & $\mathrm{Pd}(\mathrm{OAc})_{2}(0.1)$ & $\mathrm{BQ}(0.5$ equiv $)+\mathrm{Ar}$ & DMSO (2) & 28 \\
\hline 5 & $\mathrm{Pd}(\mathrm{OAc})_{2}(0.1)$ & $\mathrm{Ar}$ & DMSO (2) & NR \\
\hline 6 & $\mathrm{Pd}(\mathrm{OAc})_{2}(0.1)$ & $\mathrm{BQ}(1.0$ equiv $)+\mathrm{O}_{2}$ & DMSO (2) & 46 \\
\hline 7 & $\operatorname{Pd}(\mathrm{OAc})_{2}(0.1)$ & $\mathrm{BQ}(2.0$ equiv $)+\mathrm{O}_{2}$ & DMSO (2) & 67 \\
\hline 8 & $\mathrm{Pd}(\mathrm{OAc})_{2}(0.1)$ & $\mathrm{BQ}(3.0$ equiv $)+\mathrm{O}_{2}$ & DMSO (2) & 63 \\
\hline
\end{tabular}

${ }^{a}$ Reaction conditions: IV1a $(0.5 \mathrm{mmol})$, cat. $(0.05 \mathrm{mmol})$, oxidant in indicated, solvent $(2.0 \mathrm{~mL})$ was stirred at $40{ }^{\circ} \mathrm{C}$ for $12 \mathrm{~h} .{ }^{b}$ The yields were determined by LC analysis using biphenyl as the internal standard.

Table S2: The reaction with different solvent and catalyst. ${ }^{a}$

\begin{tabular}{|c|c|c|c|c|}
\hline entry & $\begin{array}{c}\text { cat. } \\
\text { (equiv) }\end{array}$ & oxidant & solvent $(\mathrm{mL})$ & $\begin{array}{l}\text { Yield }(\%)^{b} \\
(\text { LC-MS) }\end{array}$ \\
\hline 1 & $\mathrm{Pd}(\mathrm{OAc})_{2}(0.1)$ & $\mathrm{BQ}(2.0$ equiv $)+\mathrm{O}_{2}$ & DMF (2) & 18 \\
\hline 2 & $\mathrm{Pd}(\mathrm{OAc})_{2}(0.1)$ & $\mathrm{BQ}(2.0$ equiv $)+\mathrm{O}_{2}$ & DMA (2) & 15 \\
\hline 3 & $\mathrm{Pd}(\mathrm{OAc})_{2}(0.1)$ & $\mathrm{BQ}(2.0$ equiv $)+\mathrm{O}_{2}$ & 1,4-dioxane (2) & Trace \\
\hline 4 & $\mathrm{Pd}(\mathrm{OAc})_{2}(0.1)$ & $\mathrm{BQ}(2.0$ equiv $)+\mathrm{O}_{2}$ & DCE (2) & Trace \\
\hline 5 & $\mathrm{Pd}(\mathrm{OAc})_{2}(0.1)$ & $\mathrm{BQ}(2.0$ equiv $)+\mathrm{O}_{2}$ & $\mathrm{EtOH}(2)$ & Trace \\
\hline 6 & $\mathrm{Pd}(\mathrm{OAc})_{2}(0.1)$ & $\mathrm{BQ}(2.0$ equiv $)+\mathrm{O}_{2}$ & $\mathrm{CH}_{3} \mathrm{CN}(2)$ & Trace \\
\hline 7 & $\operatorname{Pd}(\mathrm{TFA})_{2}(0.1)$ & $\mathrm{BQ}(2.0$ equiv $)+\mathrm{O}_{2}$ & DMSO (2) & 19 \\
\hline 8 & $\mathrm{PdCl}_{2}(0.1)$ & $\mathrm{BQ}(2.0$ equiv $)+\mathrm{O}_{2}$ & DMSO (2) & Trace \\
\hline 9 & $\mathrm{Pd}\left(\mathrm{CH}_{3} \mathrm{CN}\right)_{2} \mathrm{Cl}_{2}(0.1)$ & $\mathrm{BQ}(2.0$ equiv $)+\mathrm{O}_{2}$ & DMSO (2) & Trace \\
\hline 10 & $\operatorname{Pd}_{2}(\mathrm{dba})_{3}(0.1)$ & $\mathrm{BQ}(2.0$ equiv $)+\mathrm{O}_{2}$ & DMSO (2) & 16 \\
\hline 11 & $\mathrm{Pd}\left(\mathrm{PPh}_{3}\right)_{4}(0.1)$ & $\mathrm{BQ}(2.0$ equiv $)+\mathrm{O}_{2}$ & DMSO (2) & 10 \\
\hline 12 & $\operatorname{Pd}(\mathrm{OAc})_{2}(0.1)$ & $\mathrm{BQ}(2.0$ equiv $)+\mathrm{O}_{2}$ & DMSO (2) & $90(86)^{c}$ \\
\hline
\end{tabular}

${ }^{a}$ Reaction conditions: IV1a $(0.5 \mathrm{mmol})$, cat. $(0.05 \mathrm{mmol})$, oxidant in indicated, solvent $(2.0 \mathrm{~mL})$ was stirred at $40{ }^{\circ} \mathrm{C}$ for $12 \mathrm{~h}$. ${ }^{b}$ The yields were determined by LC analysis using biphenyl as the internal standard. ${ }^{c}$ The reaction was stirred at $60{ }^{\circ} \mathrm{C}$ with $\mathrm{Pd}(\mathrm{OAc})_{2}(0.025 \mathrm{mmol})$ for $6 \mathrm{~h}$, then added $\mathrm{Pd}(\mathrm{OAc})_{2}(0.025 \mathrm{mmol})$ and stirred at $60{ }^{\circ} \mathrm{C}$ for another $6 \mathrm{~h}$. 


\section{Scheme S1. Control experiments.}

We examined the annulation of ortho-alkynyl biphenyl and ortho-ethynylanilide under the standard conditions (Scheme S1). It was found that the reaction of ortho-alkynyl biphenyl $\mathbf{3}$ (or treated with methyl acrylate) did not proceed at all, and the starting material was recovered (Scheme S1, eq 1 and 2), indicating that the potential 5-exo-dig cyclization of 1a could be excluded. In contrast, the protonated product $\mathbf{7}$ and cross-coupling product $\mathbf{8}$ were obtained in $58 \%$ and $79 \%$ yield respectively when ortho-ethynylanilide 6 was applied under a corresponding reaction conditions (Scheme S1, eq 3 and 4). This result implies the involvement of a 6-exo-dig cyclization and a 1,3-oxazine vinylpalladium intermediate.

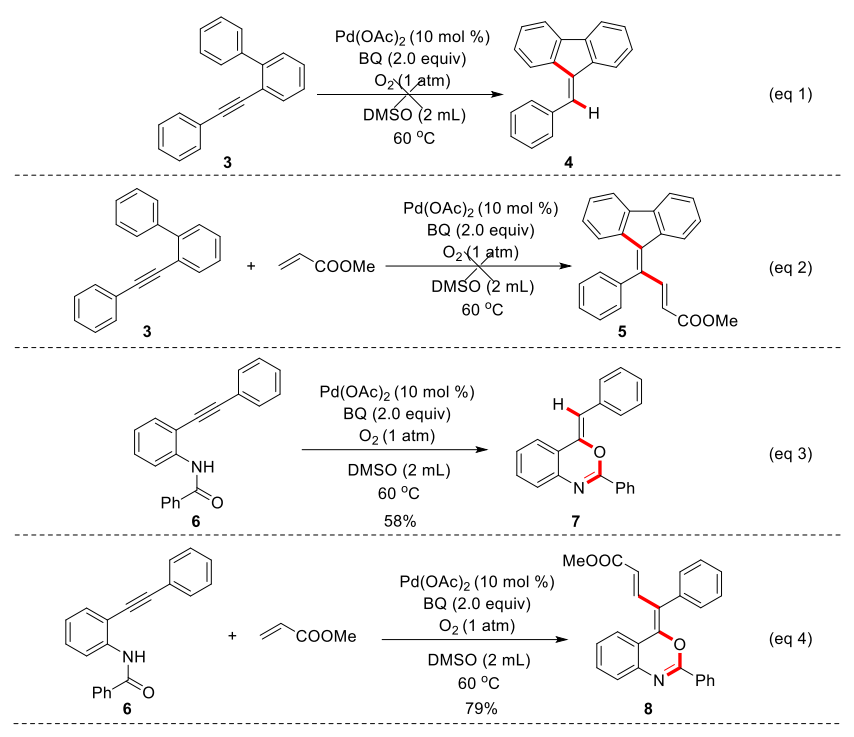




\section{Characterization Data of Compounds of 2a-2t and 2a':}

\section{4-(9H-fluoren-9-ylidene)-2-phenyl-4H-benzo[d][1,3]oxazine (2a)}

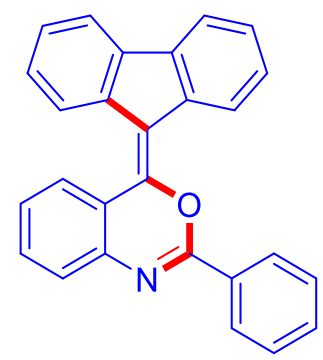

$159.5 \mathrm{mg}$ (yield, 86\%); Yellow Solid, mp: $151-152{ }^{\circ} \mathrm{C}$;

IR (neat, $v, \mathrm{~cm}-1)$ : 1628, 1586, 1442, 765, 724, $684 \mathrm{~cm}^{-1}$;

${ }^{1} \mathbf{H}$ NMR (400 MHz, CDCl$) \delta 8.46(\mathrm{~d}, J=7.0 \mathrm{~Hz}, 1 \mathrm{H}), 8.27(\mathrm{~d}, J=7.0 \mathrm{~Hz}$, 2H), $8.09(\mathrm{~d}, J=7.6 \mathrm{~Hz}, 1 \mathrm{H}), 7.96(\mathrm{~d}, J=8.0 \mathrm{~Hz}, 1 \mathrm{H}), 7.80(\mathrm{~d}, J=6.6 \mathrm{~Hz}$, $1 \mathrm{H}), 7.75(\mathrm{~d}, J=7.5 \mathrm{~Hz}, 1 \mathrm{H}), 7.57-7.44(\mathrm{~m}, 5 \mathrm{H}), 7.42-7.34(\mathrm{~m}, 2 \mathrm{H}), 7.33$ $-7.24(\mathrm{~m}, 2 \mathrm{H}), 7.11(\mathrm{t}, J=7.4 \mathrm{~Hz}, 1 \mathrm{H})$;

${ }^{13}$ C NMR (100 MHz, CDCl 3$) \delta 156.04,148.44,142.35,139.63,137.91$, 137.00, 136.04, 132.29, 131.67, 130.85, 128.89, 128.24, 128.16, 126.74, 126.57, 126.54, 126.48, 125.81, 125.62, 124.84, 122.02, 120.23, 119.40, 119.08, 116.90, 76.93, 76.61, 76.29.

HRMS (ESI) $m / z$ : Found: 372.1392. Calcd for $\mathrm{C}_{27} \mathrm{H}_{17} \mathrm{NO}:(\mathrm{M}+\mathrm{H})^{+} 372.1383$.

(E)-4-(2-fluoro-9H-fluoren-9-ylidene)-2-phenyl-4H-benzo[d][1,3]oxazine (2b)

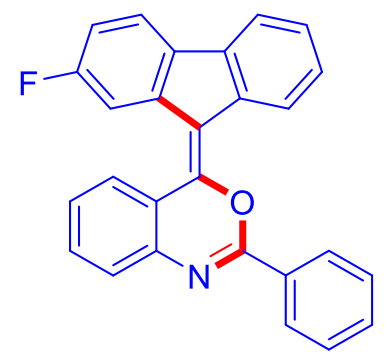

$153.6 \mathrm{mg}$ (yield, 79\%); Yellow Solid, mp: $195-196{ }^{\circ} \mathrm{C}$

IR (neat, $v, \mathrm{~cm}-1)$ : $1628,1571,1449,755,688 \mathrm{~cm}^{-1}$;

${ }^{1} \mathbf{H}_{\text {NMR }}{ }^{1} \mathbf{H}$ NMR (400 MHz, $\left.\mathbf{C D C l}_{3}\right) \delta 8.45-8.42(\mathrm{~m}, 1 \mathrm{H}), 8.27$ (dd, $J$ $=5.2,3.3 \mathrm{~Hz}, 2 \mathrm{H}), 8.06(\mathrm{dd}, J=7.9,1.0 \mathrm{~Hz}, 1 \mathrm{H}), 7.76-7.72(\mathrm{~m}, 1 \mathrm{H})$, $7.70-7.62(\mathrm{~m}, 2 \mathrm{H}), 7.58(\mathrm{dd}, J=11.3,4.1 \mathrm{~Hz}, 2 \mathrm{H}), 7.51(\mathrm{dd}, J=10.2$, $4.8 \mathrm{~Hz}, 3 \mathrm{H}), 7.39-7.34(\mathrm{~m}, 3 \mathrm{H}), 6.98(\mathrm{td}, J=8.6,2.3 \mathrm{~Hz}, 1 \mathrm{H})$;

${ }^{13}$ C NMR (100 MHz, $\left.\mathbf{C D C l}_{3}\right) \delta 161.53(J=240.2 \mathrm{~Hz}), 155.81,149.43$, $142.42,137.73(J=9.1 \mathrm{~Hz}), 137.09,137.01,135.65,132.75,131.76,130.64,128.61,128.25$, $128.14,126.74,126.67,126.36,125.83,124.68,120.11(\mathrm{~J}=9.2 \mathrm{~Hz}), 119.70,118.75,116.38(\mathrm{~J}=$ $3.2 \mathrm{~Hz}), 113.56(\mathrm{~J}=23.4 \mathrm{~Hz}), 108.98(\mathrm{~J}=25.4 \mathrm{~Hz}), 76.88,76.56,76.25$;

HRMS (ESI) $m / z$ : Found: 390.1281. Calcd for $\mathrm{C}_{27} \mathrm{H}_{16} \mathrm{FNO}:(\mathrm{M}+\mathrm{H})^{+} 390.1289$.

(E)-4-(4-chloro-9H-fluoren-9-ylidene)-2-phenyl-4H-benzo[d][1,3]oxazine (2c)

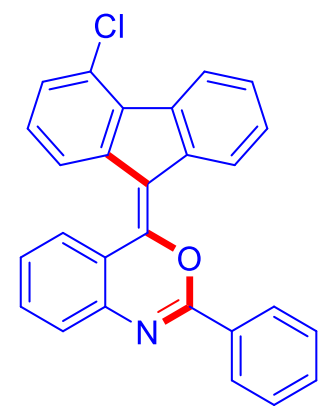

$170.1 \mathrm{mg}$ (yield, 84\%); Yellow Solid, mp: $221-222{ }^{\circ} \mathrm{C}$

IR (neat, $v, \mathrm{~cm}-1)$ : 1628, 1572, 1447, 722, $687 \mathrm{~cm}^{-1}$;

${ }^{1}$ H NMR (400 MHz, CDCl $) \delta 8.54(\mathrm{dd}, J=41.5,21.9 \mathrm{~Hz}, 2 \mathrm{H}), 8.29(\mathrm{~d}, J=$ $5.1 \mathrm{~Hz}, 2 \mathrm{H}), 8.19-7.83(\mathrm{~m}, 2 \mathrm{H}), 7.63-7.22(\mathrm{~m}, 9 \mathrm{H}), 7.11(\mathrm{~d}, J=56.5 \mathrm{~Hz}$, $1 \mathrm{H})$;

${ }^{13}$ C NMR (100 MHz, $\left.\mathbf{C D C l}_{3}\right) \delta$ 155.83, 149.38, 142.52, 138.70, 137.25, $136.55,135.54,132.66,131.81,130.61,129.10,128.51,128.29,128.15$, $127.96,127.07,126.58,126.55,125.93,125.80,124.21,123.26,120.04$, 119.93, 116.16, 76.87, 76.55, 76.23;

HRMS (ESI) $m / z$ : Found: 406.0983; Calcd for $\mathrm{C}_{27} \mathrm{H}_{16} \mathrm{ClNO}(\mathrm{M}+\mathrm{H})^{+} 406.0993$. 


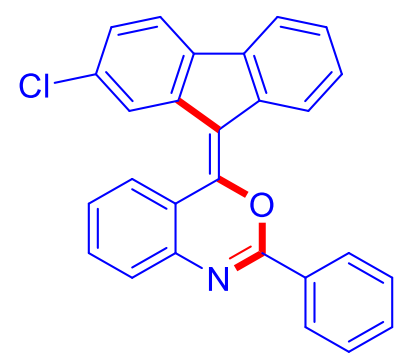

$146.2 \mathrm{mg}$ (yield, $72 \%$ ); Yellow Solid, mp: $198-199^{\circ} \mathrm{C}$

IR (neat, $v, \mathrm{~cm}-1)$ : 1630, 1573, 1438, 724, $685 \mathrm{~cm}^{-1}$;

${ }^{1}$ H NMR (400 MHz, CDCl $) \delta 8.51-8.41(\mathrm{~m}, 1 \mathrm{H}), 8.28(\mathrm{~d}, J=7.4 \mathrm{~Hz}$, $2 \mathrm{H}), 8.06(\mathrm{~d}, J=7.7 \mathrm{~Hz}, 1 \mathrm{H}), 7.94(\mathrm{~d}, J=10.3 \mathrm{~Hz}, 1 \mathrm{H}), 7.75(\mathrm{t}, J=10.5$ $\mathrm{Hz}, 1 \mathrm{H}), 7.71-7.63(\mathrm{~m}, 1 \mathrm{H}), 7.62-7.48(\mathrm{~m}, 5 \mathrm{H}), 7.46-7.30(\mathrm{~m}, 3 \mathrm{H})$, $7.27-7.18(\mathrm{~m}, 1 \mathrm{H})$;

${ }^{13}$ C NMR (101 MHz, CDCl $) \delta 155.75,149.50,142.44,137.86,137.56$, 136.91, 136.83, 132.85, 131.77, 131.48, 130.63, 128.74, 128.26, 128.14, 126.93, 126.72, 126.64, $126.35,125.85,124.69,121.99,120.12,119.66,119.07,115.89,76.87,76.55,76.24$

HRMS (ESI) $m / z$ : Found: 406.0997; Calcd for $\mathrm{C}_{27} \mathrm{H}_{16} \mathrm{ClNO}:(\mathrm{M}+\mathrm{H})^{+} 406.0993$.

\section{(E)-4-(2-methoxy-9H-fluoren-9-ylidene)-2-phenyl-4H-benzo[d][1,3]oxazine (2e)}

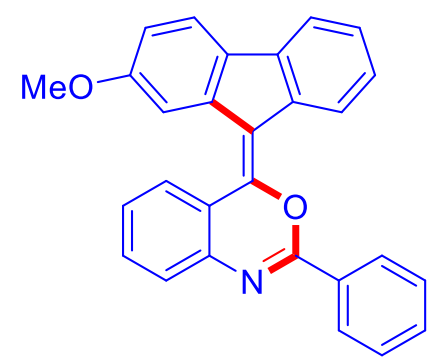

$146.7 \mathrm{mg}$ (yield, $73 \%$ ); Yellow Solid, mp: $171-172{ }^{\circ} \mathrm{C}$

IR (neat, $v, \mathrm{~cm}-1): 1628,1587,1452,769,727,679 \mathrm{~cm}^{-1}$;

${ }^{1} \mathbf{H}$ NMR (400 MHz, $\left.\mathbf{C D C l}_{3}\right) \delta 8.39(\mathrm{dd}, J=6.0,2.8 \mathrm{~Hz}, 1 \mathrm{H}), 8.29-$ $8.23(\mathrm{~m}, 2 \mathrm{H}), 8.13(\mathrm{~d}, J=7.5 \mathrm{~Hz}, 1 \mathrm{H}), 7.66(\mathrm{dd}, J=5.9,2.7 \mathrm{~Hz}, 1 \mathrm{H})$, $7.60(\mathrm{~d}, J=8.4 \mathrm{~Hz}, 1 \mathrm{H}), 7.54-7.44(\mathrm{~m}, 6 \mathrm{H}), 7.34-7.26(\mathrm{~m}, 3 \mathrm{H})$, $6.83(\mathrm{dd}, J=8.4,2.2 \mathrm{~Hz}, 1 \mathrm{H}), 3.69(\mathrm{~s}, 3 \mathrm{H})$;

${ }^{13}$ C NMR (100 MHz, CDCl $) \delta 158.29,156.07,148.44,142.41$, $138.05,137.50,136.84,133.16,132.43,131.67,130.81,128.66,128.22,128.16,126.66,126.23$, $125.68,125.65,124.73,120.07,119.99,118.31,117.14,113.19,107.41,76.94,76.62,76.30$, 54.95;

HRMS (ESI) $m / z$ : Found: 402.1490. Calcd for $\mathrm{C}_{28} \mathrm{H}_{19} \mathrm{NO}_{2}:(\mathrm{M}+\mathrm{H})^{+} 402.1489$.

\section{(E)-4-(2-methyl-9H-fluoren-9-ylidene)-2-phenyl-4H-benzo[d][1,3]oxazine (2f)}

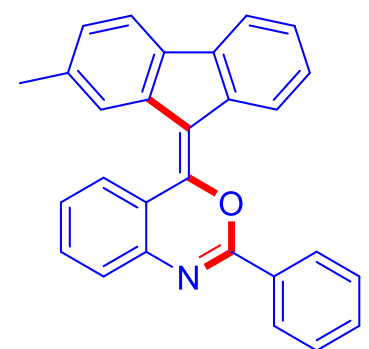

$142.8 \mathrm{mg}$ (yield, $74 \%$ ); Yellow Solid, mp: $185-186{ }^{\circ} \mathrm{C}$

IR (neat, $v, \mathrm{~cm}-1)$ : 1629, 1569, 1448, 759, $687 \mathrm{~cm}^{-1}$;

${ }^{1} \mathbf{H}$ NMR (400 MHz, CDCl $) \delta 8.47-8.39(\mathrm{~m}, 1 \mathrm{H}), 8.26(\mathrm{~d}, J=7.2 \mathrm{~Hz}$, $2 \mathrm{H}), 8.10(\mathrm{~d}, J=7.8 \mathrm{~Hz}, 1 \mathrm{H}), 7.78(\mathrm{~s}, 1 \mathrm{H}), 7.76-7.71(\mathrm{~m}, 1 \mathrm{H}), 7.61(\mathrm{~d}, J$ $=7.7 \mathrm{~Hz}, 1 \mathrm{H}), 7.50(\mathrm{tt}, J=14.2,7.1 \mathrm{~Hz}, 5 \mathrm{H}), 7.35(\mathrm{dd}, J=9.2,5.8 \mathrm{~Hz}$, $2 \mathrm{H}), 7.29(\mathrm{t}, J=7.4 \mathrm{~Hz}, 1 \mathrm{H}), 2.29(\mathrm{~s}, 3 \mathrm{H})$;

${ }^{13}$ C NMR (100 MHz, $\left.\mathbf{C D C l}_{3}\right) \delta 156.11,148.13,142.36,138.08,137.28$, 137.00, 136.28, 135.47, 132.27, 131.65, 130.89, 128.90, 128.23, 128.17, 127.61, 126.56, 126.28, 126.26, 125.58, 124.83, 122.60, 120.30, 119.12, 118.76, 117.00, 76.94, 76.63, 76.31, 21.46;

HRMS (ESI) $m / z$ : Found: 386.1549. Calcd for $\mathrm{C}_{28} \mathrm{H}_{19} \mathrm{NO}:(\mathrm{M}+\mathrm{H})^{+} 386.1539$. 
(E)-4-(4-methyl-9H-fluoren-9-ylidene)-2-phenyl-4H-benzo[d][1,3]oxazine (2g)

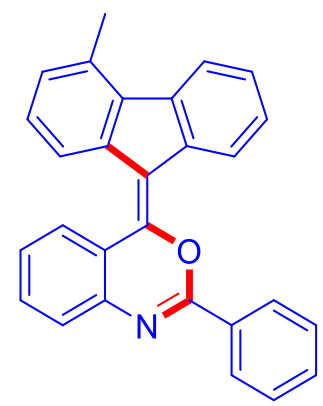

$154.4 \mathrm{mg}$ (yield, $80 \%$ ); Yellow Solid, mp: $161-162{ }^{\circ} \mathrm{C}$

IR (neat, v, cm-1): 1627, 1574, 768, 724, $688 \mathrm{~cm}^{-1}$;

${ }^{1} \mathbf{H}$ NMR (400 MHz, CDCl $) \delta 8.58-8.52(\mathrm{~m}, 1 \mathrm{H}), 8.32-8.27(\mathrm{~m}, 2 \mathrm{H})$, $8.12(\mathrm{~d}, J=7.8 \mathrm{~Hz}, 1 \mathrm{H}), 7.99(\mathrm{dd}, J=5.6,3.1 \mathrm{~Hz}, 1 \mathrm{H}), 7.88(\mathrm{~d}, J=7.6 \mathrm{~Hz}$, $1 \mathrm{H}), 7.59-7.48(\mathrm{~m}, 5 \mathrm{H}), 7.44-7.37(\mathrm{~m}, 2 \mathrm{H}), 7.33-7.28(\mathrm{~m}, 1 \mathrm{H}), 7.09-$ $6.98(\mathrm{~m}, 2 \mathrm{H}), 2.74(\mathrm{~s}, 3 \mathrm{H})$;

${ }^{13}$ C NMR (100 MHz, CDCl 3$) \delta 156.08,148.18,142.40,138.90,137.53$, $137.41,136.50,132.48,132.22,131.66,130.85,129.19,129.11,128.24$, $128.16,126.51,126.43,126.00,125.57,125.27,124.57,122.58,120.40,119.57,117.12,76.89$, 76.57, 76.25, 21.10;

HRMS (ESI) $m / z$ : Found: 386.1537. Calcd for $\mathrm{C}_{28} \mathrm{H}_{19} \mathrm{NO}:(\mathrm{M}+\mathrm{H})^{+} 386.1539$.

(E)-4-(3-methyl-9H-fluoren-9-ylidene)-2-phenyl-4H-benzo[d][1,3]oxazine (2h)

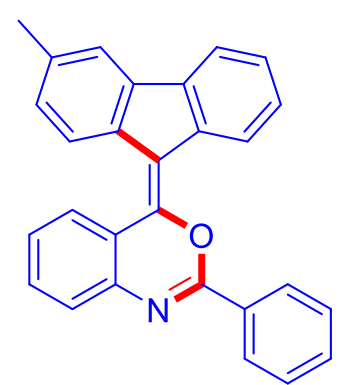

$169.8 \mathrm{mg}$ (yield, $88 \%$ ); Yellow Solid, mp: $193-194{ }^{\circ} \mathrm{C}$

IR (neat, $v, \mathrm{~cm}-1)$ : 1630, 1573, 1442, 760, $690 \mathrm{~cm}^{-1}$;

${ }^{1} \mathbf{H}$ NMR $\left(400 \mathrm{MHz}, \mathbf{C D C l}_{3}\right) \delta 8.42(\mathrm{~d}, J=7.0 \mathrm{~Hz}, 1 \mathrm{H}), 8.24(\mathrm{~d}, J=7.2 \mathrm{~Hz}$, $2 \mathrm{H}), 8.05(\mathrm{~d}, J=7.8 \mathrm{~Hz}, 1 \mathrm{H}), 7.81(\mathrm{~d}, J=8.1 \mathrm{~Hz}, 1 \mathrm{H}), 7.77-7.71(\mathrm{~m}, 1 \mathrm{H})$, $7.56-7.42(\mathrm{~m}, 6 \mathrm{H}), 7.33(\mathrm{dd}, J=13.4,7.1 \mathrm{~Hz}, 2 \mathrm{H}), 7.25(\mathrm{t}, J=7.3 \mathrm{~Hz}, 1 \mathrm{H})$, $6.91(\mathrm{~d}, J=8.0 \mathrm{~Hz}, 1 \mathrm{H}), 2.39(\mathrm{~s}, 3 \mathrm{H})$;

${ }^{13}$ C NMR (101 MHz, CDCl $) \delta 156.12,147.60,142.28,139.94,137.98$, $137.36,136.50,133.38,132.04,131.61,130.94,128.79,128.21,128.15$, $126.85,126.63,126.52,126.38,125.54,124.91,121.87,120.42,119.96,118.96,116.92,76.96$, $76.65,76.33,21.17$

HRMS (ESI) $m / z$ : Found: 386.1533. Calcd for $\mathrm{C}_{28} \mathrm{H}_{19} \mathrm{NO}:(\mathrm{M}+\mathrm{H})^{+} 386.1539$.

\section{4-(9H-fluoren-9-ylidene)-2-(p-tolyl)-4H-benzo[d][1,3]oxazine (2i)}

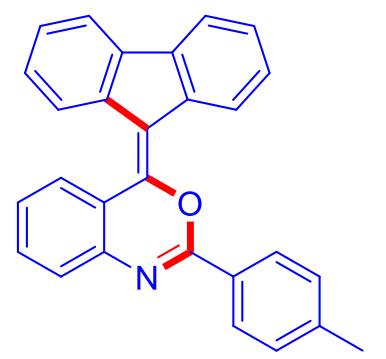

$169.1 \mathrm{mg}$ (yield, $88 \%$ ); Yellow Solid, mp: $178-179{ }^{\circ} \mathrm{C}$

IR (neat, $v, \mathrm{~cm}-1)$ : $1627,1582,1438,754,720 \mathrm{~cm}^{-1}$;

${ }^{1}$ H NMR (400 MHz, CDCl $) \delta 8.44(\mathrm{~d}, J=7.2 \mathrm{~Hz}, 1 \mathrm{H}), 8.14(\mathrm{~d}, J=8.0$ $\mathrm{Hz}, 2 \mathrm{H}), 8.07(\mathrm{~d}, J=7.7 \mathrm{~Hz}, 1 \mathrm{H}), 7.95(\mathrm{~d}, J=7.9 \mathrm{~Hz}, 1 \mathrm{H}), 7.77(\mathrm{~d}, J=$ $6.8 \mathrm{~Hz}, 1 \mathrm{H}), 7.73(\mathrm{~d}, J=7.5 \mathrm{~Hz}, 1 \mathrm{H}), 7.53-7.41(\mathrm{~m}, 2 \mathrm{H}), 7.40-7.32(\mathrm{~m}$, 2H), 7.25 (dd, $J=12.6,7.4 \mathrm{~Hz}, 4 \mathrm{H}), 7.10(\mathrm{t}, J=7.5 \mathrm{~Hz}, 1 \mathrm{H}), 2.39$ (s, 3H);

${ }^{13}$ C NMR (100 MHz, CDCl 3$) \delta 156.14,148.64,142.54,142.29,139.58$,

137.86, 137.05, 136.11, 132.26, 128.98, 128.90, 128.17, 128.03, 126.68, 126.48, 126.46, 126.24, $125.78,125.52,124.83,122.02$, 120.17, 119.38, 119.06, 116.72, 76.96, 76.64, 76.33, 21.24;

HRMS (ESI) $m / z$ : Found: 386.1546. Calcd for $\mathrm{C}_{28} \mathrm{H}_{19} \mathrm{NO}:(\mathrm{M}+\mathrm{H})^{+} 386.1539$. 


\section{2-(4-ethylphenyl)-4-(9H-fluoren-9-ylidene)-4H-benzo[d][1,3]oxazine (2j)}

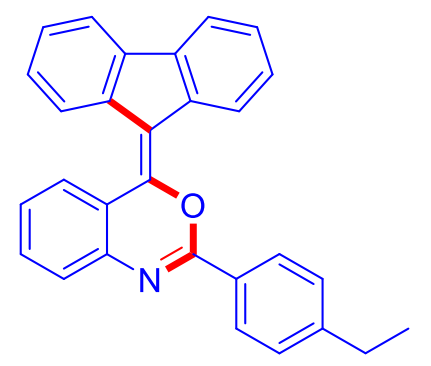

$174.1 \mathrm{mg}$ (yield, 87\%); Yellow Solid, mp: $137-138{ }^{\circ} \mathrm{C}$;

IR (neat, $v, \mathrm{~cm}-1)$ : $1627,1584,1439,757,724 \mathrm{~cm}^{-1}$;

${ }^{1} \mathbf{H}$ NMR (400 MHz, CDCl $) \delta 8.45(\mathrm{~d}, J=7.4 \mathrm{~Hz}, 1 \mathrm{H}), 8.17(\mathrm{~d}, J=$ $8.1 \mathrm{~Hz}, 2 \mathrm{H}), 8.07(\mathrm{~d}, J=7.7 \mathrm{~Hz}, 1 \mathrm{H}), 7.95(\mathrm{~d}, J=7.9 \mathrm{~Hz}, 1 \mathrm{H}), 7.78(\mathrm{~d}$, $J=7.0 \mathrm{~Hz}, 1 \mathrm{H}), 7.73(\mathrm{~d}, J=7.5 \mathrm{~Hz}, 1 \mathrm{H}), 7.53-7.42(\mathrm{~m}, 2 \mathrm{H}), 7.37(\mathrm{dd}$, $J=16.0,7.5 \mathrm{~Hz}, 2 \mathrm{H}), 7.32-7.22(\mathrm{~m}, 4 \mathrm{H}), 7.10(\mathrm{t}, J=7.5 \mathrm{~Hz}, 1 \mathrm{H})$, $2.70(\mathrm{q}, J=7.5 \mathrm{~Hz}, 2 \mathrm{H}), 1.26(\mathrm{t}, J=7.6 \mathrm{~Hz}, 3 \mathrm{H})$;

${ }^{13}$ C NMR (101 MHz, CDCl $) \delta 156.16,148.65,148.49,142.56,139.59,137.86,137.06,136.12$, $132.26,128.90,128.29,128.25,127.79,126.70,126.49,126.46,126.24,125.78,125.54,124.86$, $122.03,120.18,119.39,119.06,116.73,76.97,76.65,76.33,28.52,14.81$;

HRMS (ESI) $m / z$ : Found: 400.1694. Calcd for $\mathrm{C}_{29} \mathrm{H}_{21} \mathrm{NO}:(\mathrm{M}+\mathrm{H})^{+} 400.1696$.

\section{4-(9H-fluoren-9-ylidene)-2-(m-tolyl)-4H-benzo[d][1,3]oxazine (2k)}

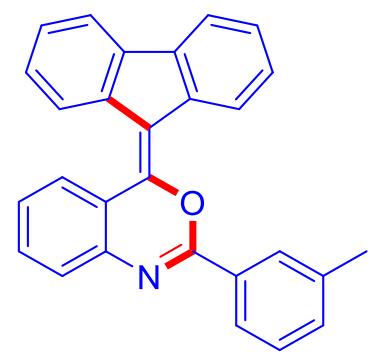

$137.1 \mathrm{mg}$ (yield, $71 \%$ ); Yellow Solid, mp: $141-142{ }^{\circ} \mathrm{C}$

IR (neat, $v, \mathrm{~cm}-1)$ : 1631, 1576, 1443, 758, $727 \mathrm{~cm}^{-1}$;

${ }^{1} \mathbf{H}$ NMR (400 MHz, CDCl $) \delta 8.50(\mathrm{dd}, J=6.3,2.1 \mathrm{~Hz}, 1 \mathrm{H}), 8.17-8.10$ $(\mathrm{m}, 2 \mathrm{H}), 8.07(\mathrm{~d}, J=7.2 \mathrm{~Hz}, 1 \mathrm{H}), 7.98(\mathrm{~d}, J=8.0 \mathrm{~Hz}, 1 \mathrm{H}), 7.81(\mathrm{dd}, J=$ 6.0, $2.3 \mathrm{~Hz}, 1 \mathrm{H}), 7.77(\mathrm{~d}, J=7.5 \mathrm{~Hz}, 1 \mathrm{H}), 7.56-7.51(\mathrm{~m}, 1 \mathrm{H}), 7.48$ (dd, $J=7.8,1.0 \mathrm{~Hz}, 1 \mathrm{H}), 7.43-7.35(\mathrm{~m}, 4 \mathrm{H}), 7.35-7.26(\mathrm{~m}, 2 \mathrm{H}), 7.16-$ $7.10(\mathrm{~m}, 1 \mathrm{H}), 2.45(\mathrm{~s}, 3 \mathrm{H})$;

${ }^{13}$ C NMR (101 MHz, CDCl $) \delta 156.28,148.47,142.43,139.60,137.99,137.89,136.99,136.01$, $132.51,132.28,130.72,128.85,128.83,128.14,126.63,126.56,126.51,126.41,125.81,125.57$, 125.30, 124.92, 122.00, 120.23, 119.38, 119.05, 116.83, 76.90, 76.58, 76.27, 20.98;

HRMS (ESI) $m / z$ : Found: 386.1551. Calcd for $\mathrm{C}_{28} \mathrm{H}_{19} \mathrm{NO}:(\mathrm{M}+\mathrm{H})^{+} 386.1539$.

\section{4-(9H-fluoren-9-ylidene)-2-(o-tolyl)-4H-benzo[d][1,3]oxazine (2l)}

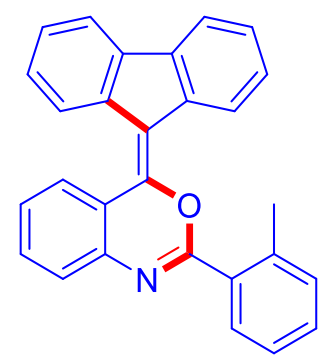

$148.6 \mathrm{mg}$ (yield, $77 \%$ ); Yellow Solid, mp: $137-138{ }^{\circ} \mathrm{C}$

IR (neat, $v, \mathrm{~cm}-1)$ : $1637,1576,1443,753,717 \mathrm{~cm}^{-1}$;

${ }^{1}$ H NMR (400 MHz, CDCl $) \delta 8.25(\mathrm{~d}, J=7.7 \mathrm{~Hz}, 1 \mathrm{H}), 8.15(\mathrm{dd}, J=7.9$, $1.1 \mathrm{~Hz}, 1 \mathrm{H}), 8.02(\mathrm{~d}, J=8.0 \mathrm{~Hz}, 1 \mathrm{H}), 7.91-7.86(\mathrm{~m}, 1 \mathrm{H}), 7.77(\mathrm{dd}, J=7.0$, $6.5 \mathrm{~Hz}, 2 \mathrm{H}), 7.57-7.51(\mathrm{~m}, 1 \mathrm{H}), 7.47(\mathrm{dd}, J=7.9,1.2 \mathrm{~Hz}, 1 \mathrm{H}), 7.45-7.40$ $(\mathrm{m}, 1 \mathrm{H}), 7.37-7.30(\mathrm{~m}, 4 \mathrm{H}), 7.30-7.22(\mathrm{~m}, 2 \mathrm{H}), 7.18-7.10(\mathrm{~m}, 1 \mathrm{H}), 2.62$ $(\mathrm{s}, 3 \mathrm{H})$;

${ }^{13}$ C NMR (100 MHz, CDCl $) \delta 156.74,148.04,142.17,139.82,138.11,137.87,137.08,135.88$, $132.22,131.05,131.04,130.58,129.44,128.55,126.81,126.70,126.64,126.61,125.79,125.57$, 125.53, 125.18, 122.20, 119.97, 119.42, 118.94, 116.86, 76.94, 76.62, 76.30, 20.46;

HRMS (ESI) $m / z$ : Found: 386.1527. Calcd for $\mathrm{C}_{28} \mathrm{H}_{19} \mathrm{NO}:(\mathrm{M}+\mathrm{H})^{+} 386.1539$. 


\section{4-(9H-fluoren-9-ylidene)-2-(4-methoxyphenyl)-4H-benzo[d][1,3]oxazine (2m)}

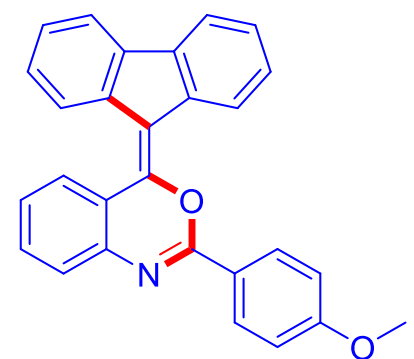

156.8 mg (yield, 78\%); Yellow Solid, mp: $193-194{ }^{\circ} \mathrm{C}$

IR (neat, v, cm-1): 1626, 1567, 1508, 767, $720 \mathrm{~cm}^{-1}$;

${ }^{1}$ H NMR (400 MHz, CDCl $) \delta 8.52-8.46(\mathrm{~m}, 1 \mathrm{H}), 8.29-8.23(\mathrm{~m}$, $2 \mathrm{H}), 8.12(\mathrm{dd}, J=7.9,1.1 \mathrm{~Hz}, 1 \mathrm{H}), 7.99(\mathrm{~d}, J=8.0 \mathrm{~Hz}, 1 \mathrm{H}), 7.86-$ $7.81(\mathrm{~m}, 1 \mathrm{H}), 7.79(\mathrm{~d}, J=7.5 \mathrm{~Hz}, 1 \mathrm{H}), 7.58-7.51(\mathrm{~m}, 1 \mathrm{H}), 7.49-7.44$ $(\mathrm{m}, 1 \mathrm{H}), 7.44-7.35(\mathrm{~m}, 2 \mathrm{H}), 7.34-7.27(\mathrm{~m}, 2 \mathrm{H}), 7.17-7.11(\mathrm{~m}, 1 \mathrm{H})$, $7.06-6.99(\mathrm{~m}, 2 \mathrm{H}), 3.89$ (s, 3H);

${ }^{13}$ C NMR (100 MHz, CDCl $) \delta 162.40,155.90,148.80,142.69,139.47,137.84,137.01,136.12$, 132.28, 130.04, 128.91, 126.67, 126.44, 126.41, 126.02, 125.79, 125.35, 124.69, 123.12, 121.97, $120.05,119.35,119.06,116.64,113.61,76.87,76.55,76.24,55.02$;

HRMS (ESI) $m / z$ : Found: 402.1480. Calcd for $\mathrm{C}_{28} \mathrm{H}_{19} \mathrm{NO}_{2}:(\mathrm{M}+\mathrm{H})^{+} 402.1489$.

\section{2-(4-chlorophenyl)-4-(9H-fluoren-9-ylidene)-4H-benzo[d][1,3]oxazine (2n)}

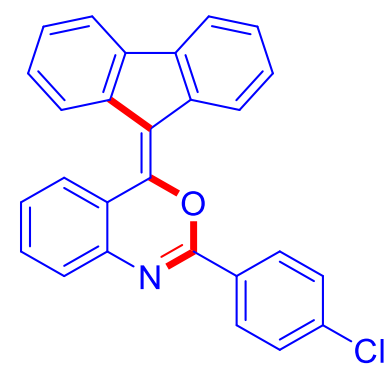

$150.3 \mathrm{mg}$ (yield, $74 \%$ ); Yellow Solid, mp: $235-236{ }^{\circ} \mathrm{C}$

IR (neat, v, cm-1): 1630, 1588, 1443, 768, $720 \mathrm{~cm}^{-1}$;

${ }^{1}$ H NMR (400 MHz, CDCl $) \delta 8.42(\mathrm{~s}, 1 \mathrm{H}), 8.24(\mathrm{~d}, J=6.3 \mathrm{~Hz}, 2 \mathrm{H})$, $8.14(\mathrm{~d}, J=6.0 \mathrm{~Hz}, 1 \mathrm{H}), 7.98(\mathrm{~d}, J=6.6 \mathrm{~Hz}, 1 \mathrm{H}), 7.88-7.72(\mathrm{~m}, 2 \mathrm{H})$, $7.62-7.45(\mathrm{~m}, 4 \mathrm{H}), 7.45-7.28(\mathrm{~m}, 4 \mathrm{H}), 7.14(\mathrm{~s}, 1 \mathrm{H})$;

${ }^{13}$ C NMR (100 MHz, $\left.\mathbf{C D C l}_{3}\right) \delta 155.13,142.09,137.98,136.86,135.90$, $132.35,129.39,128.91,128.56,126.70,125.87,125.60,124.62,121.97$, 120.22, 119.40, 119.15, 117.11, 76.85, 76.53, 76.21;

HRMS (ESI) $m / z$ : Found: 406.0999. Calcd for $\mathrm{C}_{27} \mathrm{H}_{16} \mathrm{ClNO}:(\mathrm{M}+\mathrm{H})^{+} 406.0993$.

\section{3-(4-(9H-fluoren-9-ylidene)-4H-benzo[d][1,3]oxazin-2-yl)-N,N-dimethylaniline (2o)}

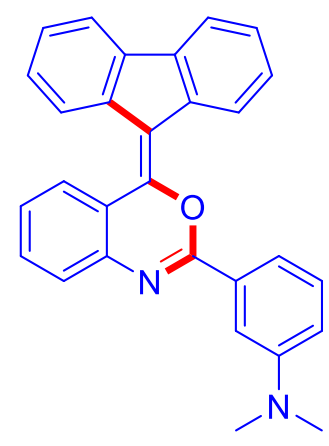

$72.7 \mathrm{mg}$ (yield, 35\%); Yellow Solid, mp: $151-152{ }^{\circ} \mathrm{C}$

IR (neat, $v, \mathrm{~cm}-1)$ : 1628, 1560, 1436, 772, $726 \mathrm{~cm}^{-1}$;

${ }^{1}$ H NMR (400 MHz, CDCl $\mathbf{l}_{3} \delta 8.60-8.54(\mathrm{~m}, 1 \mathrm{H}), 8.13(\mathrm{dd}, J=7.9,0.9$ $\mathrm{Hz}, 1 \mathrm{H}), 7.99(\mathrm{~d}, J=8.0 \mathrm{~Hz}, 1 \mathrm{H}), 7.80(\mathrm{ddd}, J=16.4,9.2,5.0 \mathrm{~Hz}, 2 \mathrm{H}), 7.64$ $(\mathrm{d}, J=7.4 \mathrm{~Hz}, 2 \mathrm{H}), 7.56-7.48(\mathrm{~m}, 2 \mathrm{H}), 7.37-7.27(\mathrm{~m}, 5 \mathrm{H}), 7.16-7.11(\mathrm{~m}$, $1 \mathrm{H}), 6.94-6.89(\mathrm{~m}, 1 \mathrm{H}), 3.02(\mathrm{~s}, 6 \mathrm{H})$;

${ }^{13}$ C NMR (100 MHz, $\left.\mathbf{C D C l}_{3}\right) \delta 156.73,150.08,148.73,142.60,139.53$, $137.83,137.08,136.10,132.27,131.43,128.93,128.84,126.56,126.48$, $126.43,126.29,125.80,125.63,124.94,122.02,120.19,119.37,119.02$, 116.57, 116.51, 115.59, 111.71, 76.91, 76.59, 76.27, 40.15;

HRMS (ESI) $m / z$ : Found: 415.1818. Calcd for $\mathrm{C}_{29} \mathrm{H}_{22} \mathrm{~N}_{2} \mathrm{O}:(\mathrm{M}+\mathrm{H})^{+} 415.1805$. 


\section{4-(9H-fluoren-9-ylidene)-2-(2-(trifluoromethyl)phenyl)-4H-benzo[d][1,3]oxazine (2p)}

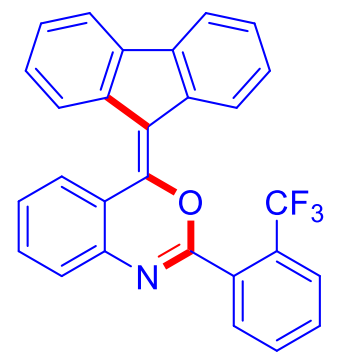

$70.4 \mathrm{mg}$ (yield, 32\%); Yellow Solid, mp: $93-94{ }^{\circ} \mathrm{C}$

IR (neat, $v, \mathrm{~cm}-1)$ : 1646, 1594, 1441, 759, $727 \mathrm{~cm}^{-1}$;

${ }^{1}$ H NMR (400 MHz, CDCl $) \delta 8.16(\mathrm{dd}, J=7.8,1.0 \mathrm{~Hz}, 1 \mathrm{H}), 8.06(\mathrm{~d}, J=$ $7.9 \mathrm{~Hz}, 1 \mathrm{H}), 8.02(\mathrm{~d}, J=7.9 \mathrm{~Hz}, 1 \mathrm{H}), 7.91-7.82(\mathrm{~m}, 2 \mathrm{H}), 7.76(\mathrm{~d}, J=7.5$ $\mathrm{Hz}, 2 \mathrm{H}), 7.71-7.63(\mathrm{~m}, 2 \mathrm{H}), 7.59-7.52(\mathrm{~m}, 1 \mathrm{H}), 7.47(\mathrm{dd}, J=7.8,0.9 \mathrm{~Hz}$, $1 \mathrm{H}), 7.42-7.36(\mathrm{~m}, 1 \mathrm{H}), 7.34-7.27(\mathrm{~m}, 2 \mathrm{H}), 7.21-7.12(\mathrm{~m}, 2 \mathrm{H})$;

${ }^{13}$ C NMR (100 MHz, CDCl $) \delta 154.87,147.48,141.64,140.04,137.97$, 137.00, 135.66, 132.27, 131.57, 130.46, 130.41, 128.77 ( $J=31.8 \mathrm{~Hz}), 128.46,127.19,126.88$, 126.77, 126.74, $126.69(J=4.9 \mathrm{~Hz}), 125.82,125.72,125.36,123.05(J=272.2 \mathrm{~Hz}), 122.33$, 120.07, 119.43, 118.86, 117.36, 76.90, 76.58, 76.27;

HRMS (ESI) $m / z$ : Found: 440.1262. Calcd for $\mathrm{C}_{28} \mathrm{H}_{16} \mathrm{~F}_{3} \mathrm{NO}:(\mathrm{M}+\mathrm{H})^{+} 440.1257$.

\section{4-(9H-fluoren-9-ylidene)-2-(naphthalen-2-yl)-4H-benzo[d][1,3]oxazine (2q)}

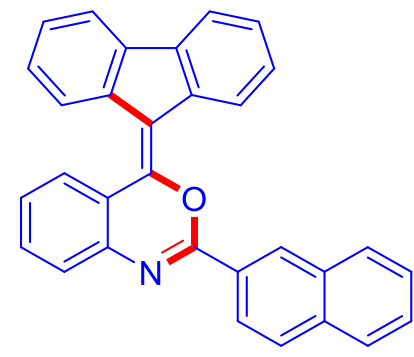

$122.4 \mathrm{mg}$ (yield, 58\%); Yellow Solid, mp: $190-191{ }^{\circ} \mathrm{C}$

IR (neat, $v, \mathrm{~cm}-1)$ : 1627, 1581, 1441, 745, $722 \mathrm{~cm}^{-1}$;

${ }^{1} \mathbf{H}$ NMR (400 MHz, CDCl $) \delta 8.82(\mathrm{~s}, 1 \mathrm{H}), 8.60(\mathrm{~d}, J=7.5 \mathrm{~Hz}, 1 \mathrm{H})$, $8.32(\mathrm{dd}, J=8.7,1.6 \mathrm{~Hz}, 1 \mathrm{H}), 8.17-8.11(\mathrm{~m}, 1 \mathrm{H}), 8.00(\mathrm{~d}, J=8.0 \mathrm{~Hz}$, $1 \mathrm{H}), 7.95(\mathrm{t}, J=7.4 \mathrm{~Hz}, 2 \mathrm{H}), 7.88(\mathrm{~d}, J=7.5 \mathrm{~Hz}, 1 \mathrm{H}), 7.84(\mathrm{~d}, J=6.9$ $\mathrm{Hz}, 1 \mathrm{H}), 7.78(\mathrm{~d}, J=7.5 \mathrm{~Hz}, 1 \mathrm{H}), 7.60-7.50(\mathrm{~m}, 4 \mathrm{H}), 7.44(\mathrm{dtd}, J=$ 21.2, 7.3, 1.1 Hz, 2H), 7.37 - 7.26 (m, 2H), $7.18-7.11(\mathrm{~m}, 1 \mathrm{H})$;

${ }^{13}$ C NMR (100 MHz, CDCl $) \delta 156.10,148.54,142.45,139.60,137.96,137.01,136.01,134.68$, 132.32, 132.28, 129.12, 128.91, 128.78, 127.99, 127.57, 127.35, 126.64, 126.54, 126.51, 126.30, 125.85, 125.66, 124.94, 124.35, 121.98, 120.29, 119.41, 119.12, 116.96, 76.89, 76.57, 76.25;

HRMS (ESI) $m / z$ : Found: 422.1530 . Calcd for $\mathrm{C}_{31} \mathrm{H}_{19} \mathrm{NO}:(\mathrm{M}+\mathrm{H})^{+} 422.1539$.

\section{(E)-4-(9H-fluoren-9-ylidene)-2-styryl-4H-benzo[d][1,3]oxazine (2r)}

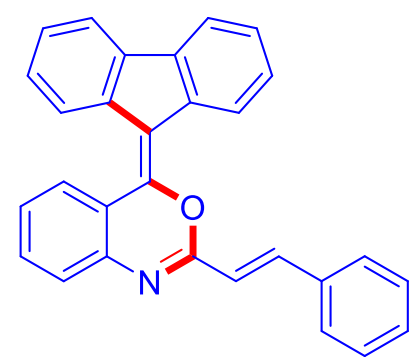

$67.7 \mathrm{mg}$ (yield, 34\%); Yellow Solid, mp: $124-125{ }^{\circ} \mathrm{C}$

IR (neat, $v, \mathrm{~cm}-1)$ : 1628, 1577, 1439, 722, $686 \mathrm{~cm}^{-1}$;

${ }^{1} \mathbf{H}$ NMR (400 MHz, CDCl $\left.\mathbf{~}_{3}\right) \delta 8.52-8.44(\mathrm{~m}, 1 \mathrm{H}), 8.12(\mathrm{dd}, J=7.9$, $1.2 \mathrm{~Hz}, 1 \mathrm{H}), 7.95(\mathrm{dd}, J=21.7,12.1 \mathrm{~Hz}, 2 \mathrm{H}), 7.86-7.76(\mathrm{~m}, 2 \mathrm{H}), 7.60$ $(\mathrm{dd}, J=7.7,1.5 \mathrm{~Hz}, 2 \mathrm{H}), 7.53(\mathrm{td}, J=7.9,1.4 \mathrm{~Hz}, 1 \mathrm{H}), 7.48-7.37(\mathrm{~m}$, $6 \mathrm{H}), 7.35-7.26(\mathrm{~m}, 2 \mathrm{H}), 7.17-7.11(\mathrm{~m}, 1 \mathrm{H}), 6.83(\mathrm{~d}, J=16.1 \mathrm{~Hz}$, $1 \mathrm{H})$;

${ }^{13}$ C NMR (100 MHz, CDCl $) \delta 155.92,148.15,142.43,140.61,139.59,137.93,137.06,135.89$, $134.47,132.27,129.63,128.81,128.54,127.48,126.62,126.54,126.42,125.82,125.34,124.88$, 122.01, 120.19, 119.38, 119.17, 119.09, 116.87, 76.87, 76.55, 76.23;

HRMS (ESI) $m / z$ : Found: 398.1538. Calcd for $\mathrm{C}_{29} \mathrm{H}_{19} \mathrm{NO}:(\mathrm{M}+\mathrm{H})^{+} 398.1539$. 


\section{4-(9H-fluoren-9-ylidene)-2-(thiophen-2-yl)-4H-benzo[d][1,3]oxazine (2s)}

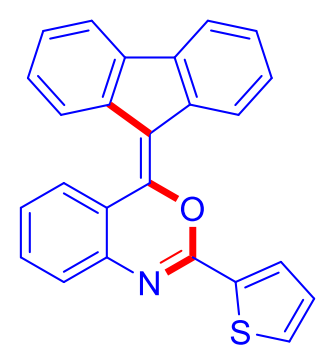

$115.3 \mathrm{mg}$ (yield, 61\%); Yellow Solid, mp: $183-184{ }^{\circ} \mathrm{C}$

IR (neat, $v, \mathrm{~cm}-1)$ : $1627,1583,1424,724,699 \mathrm{~cm}^{-1}$;

${ }^{1}$ H NMR (400 MHz, CDCl $) \delta 8.51(\mathrm{~d}, J=7.6 \mathrm{~Hz}, 1 \mathrm{H}), 8.09(\mathrm{dd}, J=7.9$, $1.1 \mathrm{~Hz}, 1 \mathrm{H}), 8.01(\mathrm{dd}, J=3.7,1.1 \mathrm{~Hz}, 1 \mathrm{H}), 7.96(\mathrm{~d}, J=8.0 \mathrm{~Hz}, 1 \mathrm{H}), 7.81(\mathrm{~d}$, $J=7.0 \mathrm{~Hz}, 1 \mathrm{H}), 7.76(\mathrm{~d}, J=7.5 \mathrm{~Hz}, 1 \mathrm{H}), 7.58(\mathrm{dd}, J=5.0,1.1 \mathrm{~Hz}, 1 \mathrm{H}), 7.55$ $-7.49(\mathrm{~m}, 1 \mathrm{H}), 7.47-7.36(\mathrm{~m}, 3 \mathrm{H}), 7.35-7.25(\mathrm{~m}, 2 \mathrm{H}), 7.18(\mathrm{dd}, J=4.9$, $3.8 \mathrm{~Hz}, 1 \mathrm{H}), 7.15-7.05(\mathrm{~m}, 1 \mathrm{H})$;

${ }^{13}$ C NMR (100 MHz, CDCl $) \delta 151.95,147.94,142.23,139.68,137.93,136.92,135.89,134.47$, $132.25,130.85,130.61,128.90,127.67,126.69,126.64,126.19,125.81,125.27,125.06,122.10$, 120.08, 119.36, 119.02, 117.13, 76.88, 76.57, 76.25;

HRMS (ESI) $m / z$ : Found: 378.0958. Calcd for $\mathrm{C}_{25} \mathrm{H}_{15} \mathrm{NOS}:(\mathrm{M}+\mathrm{H})^{+} 378.0947$.

\section{2-(tert-butyl)-4-(9H-fluoren-9-ylidene)-4H-benzo[d][1,3]oxazine (2t)}

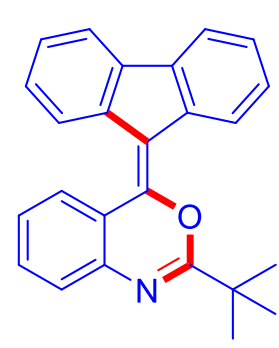

$123.3 \mathrm{mg}$ (yield, 70\%); Yellow Solid, mp: $182-183{ }^{\circ} \mathrm{C}$

IR (neat, $v, \mathrm{~cm}-1): 1634,1586,1436,755,732 \mathrm{~cm}^{-1}$;

${ }^{1}$ H NMR (400 MHz, CDCl $) \delta 8.38(\mathrm{~d}, J=7.1 \mathrm{~Hz}, 1 \mathrm{H}), 8.06(\mathrm{~d}, J=7.7 \mathrm{~Hz}$, $1 \mathrm{H}), 7.93(\mathrm{~d}, J=8.0 \mathrm{~Hz}, 1 \mathrm{H}), 7.84-7.71(\mathrm{~m}, 2 \mathrm{H}), 7.49(\mathrm{t}, J=7.3 \mathrm{~Hz}, 1 \mathrm{H})$, $7.41-7.32(\mathrm{~m}, 3 \mathrm{H}), 7.27(\mathrm{dd}, J=16.5,7.9 \mathrm{~Hz}, 2 \mathrm{H}), 7.10(\mathrm{t}, J=7.5 \mathrm{~Hz}, 1 \mathrm{H})$, $1.46(\mathrm{~s}, 9 \mathrm{H})$;

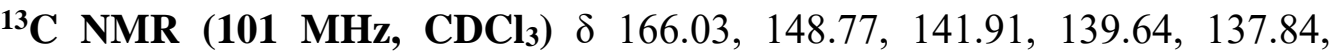
$137.05,136.10,132.14,128.73,126.60,126.42,126.16,125.75,125.30,124.99,122.02,120.04$, 119.37, 119.00, 116.32, 76.95, 76.63, 76.31, 37.33, 27.50;

HRMS (ESI) $m / z$ : Found: 352.1691. Calcd for $\mathrm{C}_{25} \mathrm{H}_{21} \mathrm{NO}:(\mathrm{M}+\mathrm{H})^{+} 352.1696$.

\section{2'-ethoxy-2'-phenyl-1',2'-dihydro-4'H-spiro[fluorene-9,3'-quinolin]-4'-one (2a')}

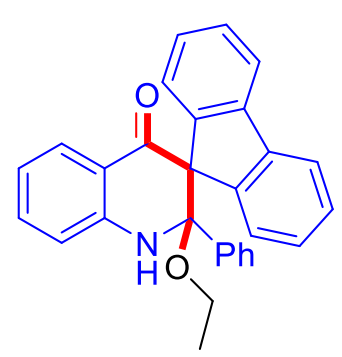

$90.0 \mathrm{mg}$ (yield, 43\%); White Solid, mp: $222-223{ }^{\circ} \mathrm{C}$

IR (neat, $v, \mathrm{~cm}-1)$ : 1657, 1604, 1479, 1445, 748, 732, $636 \mathrm{~cm}^{-1}$;

${ }^{1} \mathbf{H}$ NMR (400 MHz, DMSO-d6) $\delta 8.30(\mathrm{~s}, 1 \mathrm{H}), 7.98(\mathrm{~d}, J=7.1 \mathrm{~Hz}, 1 \mathrm{H})$, $7.70(\mathrm{dd}, J=7.9,1.4 \mathrm{~Hz}, 1 \mathrm{H}), 7.63-7.58(\mathrm{~m}, 1 \mathrm{H}), 7.56-7.51(\mathrm{~m}, 2 \mathrm{H})$, $7.47(\mathrm{t}, J=7.2 \mathrm{~Hz}, 2 \mathrm{H}), 7.41-7.27(\mathrm{~m}, 5 \mathrm{H}), 7.23(\mathrm{td}, J=7.5,1.1 \mathrm{~Hz}, 1 \mathrm{H})$, $7.02(\mathrm{td}, J=5.8,2.8 \mathrm{~Hz}, 1 \mathrm{H}), 6.94-6.87(\mathrm{~m}, 5 \mathrm{H}), 3.28(\mathrm{ddd}, J=36.9,9.4$, $7.1 \mathrm{~Hz}, 2 \mathrm{H}), 1.15(\mathrm{t}, J=7.0 \mathrm{~Hz}, 3 \mathrm{H})$;

${ }^{13}$ C NMR (100 MHz, DMSO-d6) $\delta$ 192.28, 148.35, 142.46, 141.82, 141.43, 139.51, 135.63, $135.35,129.05,128.35,127.88,127.67,127.26,126.92,126.79,126.59,126.46,125.35,119.82$, $119.19,118.29,117.80,116.31,94.41,70.97,57.00,40.13,39.92,39.71,39.51,39.30,39.09$, 38.88, 14.80;

HRMS (ESI) $m / z$ : Found: 418.1812. Calcd for $\mathrm{C}_{29} \mathrm{H}_{23} \mathrm{NO}_{2}:(\mathrm{M}+\mathrm{H})^{+} 418.1802$. 
Copies of ${ }^{1} \mathrm{H}$ and ${ }^{13} \mathrm{C}$ NMR Spectra for Compounds $2 \mathrm{a}-2 \mathrm{t}$ and $2 \mathrm{a}$ '

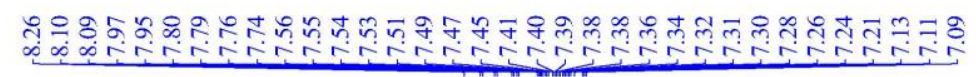

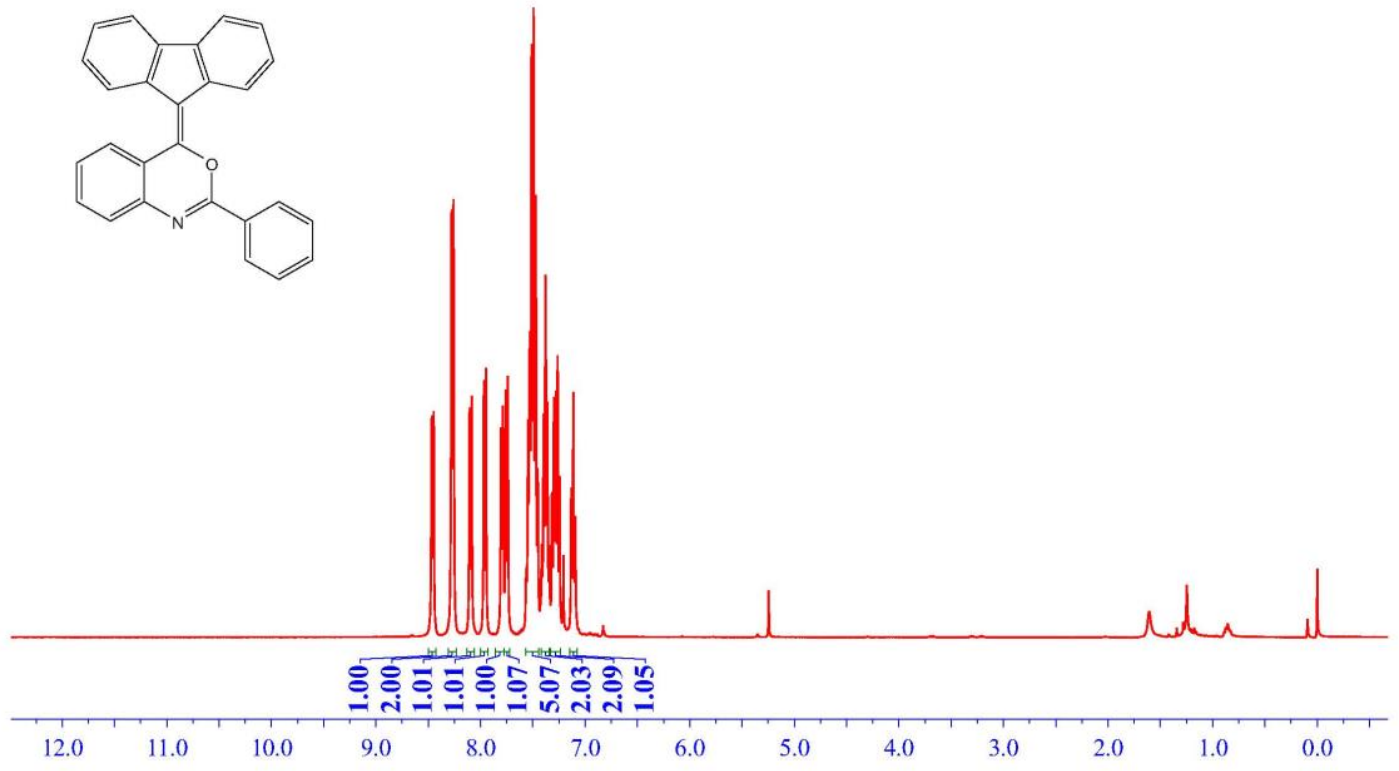

${ }^{1} H$ NMR Spectrum of Compound 2a

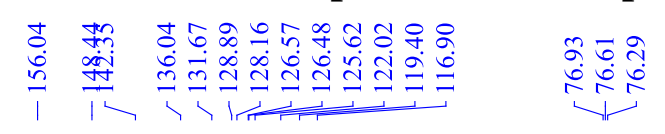

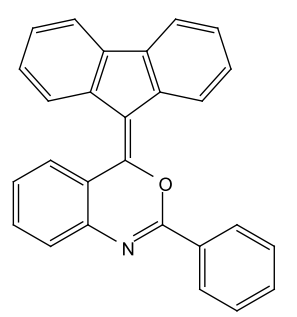

$\begin{array}{llllllllllllllllllllll}200 & 190 & 180 & 170 & 160 & 150 & 140 & 130 & 120 & 110 & 100 & 90 & 80 & 70 & 60 & 50 & 40 & 30 & 20 & 10 & 0 & -1(\end{array}$ 
${ }^{13}$ C NMR Spectrum of Compound $2 \mathrm{a}$

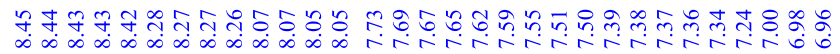

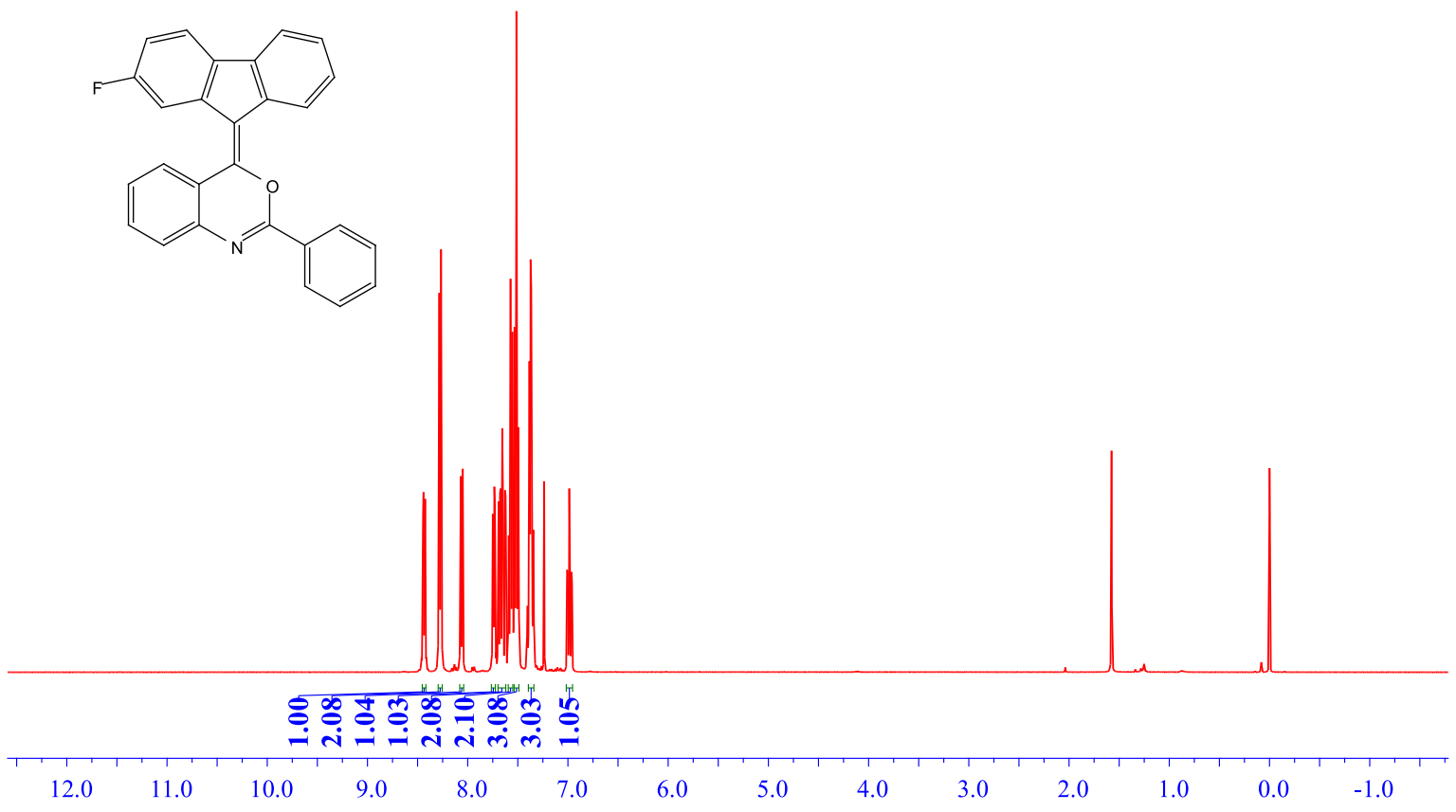

${ }^{1}$ H NMR Spectrum of Compound $2 \mathrm{~b}$

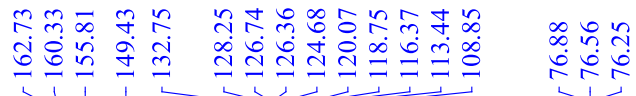
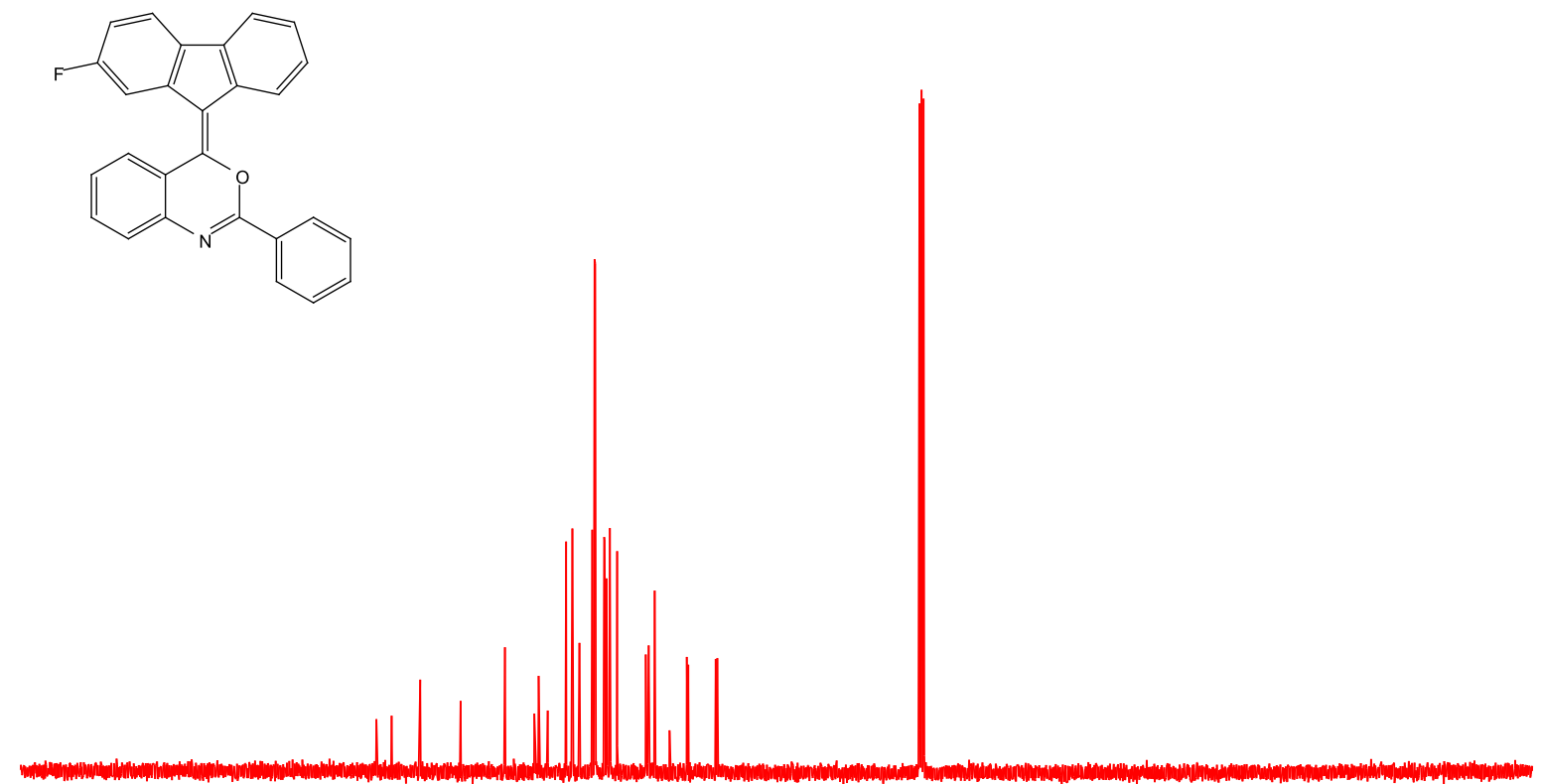

210

190

170

150

130

110

$\begin{array}{llllll}90 & 80 & 70 & 60 & 50 & 40\end{array}$

$\begin{array}{llll}20 & 10 & 0 & -10\end{array}$

${ }^{13} \mathrm{C}$ NMR Spectrum of Compound 2b 

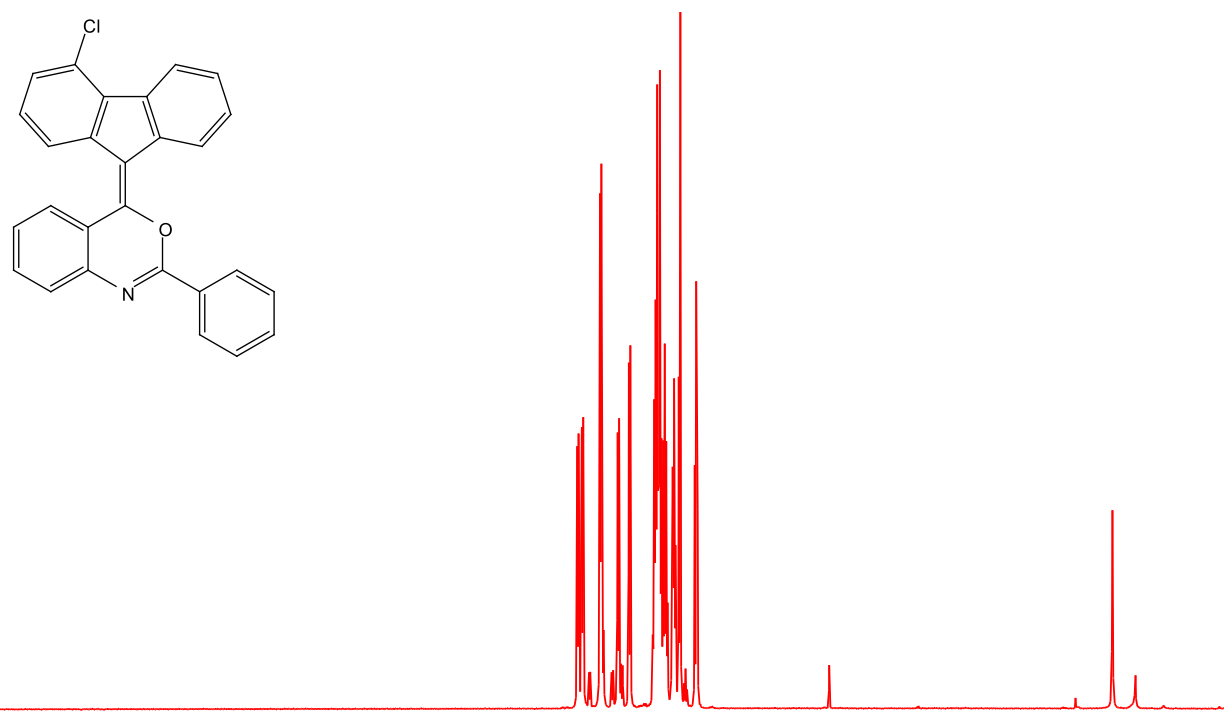

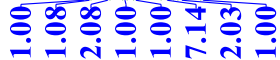

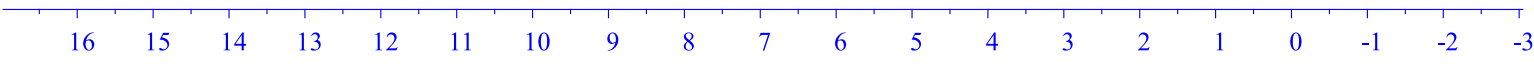

${ }^{1}$ H NMR Spectrum of Compound 2c

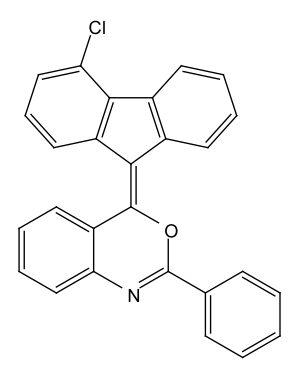

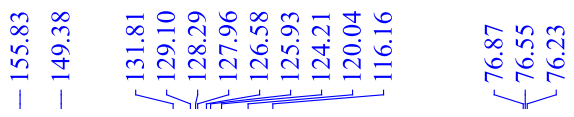

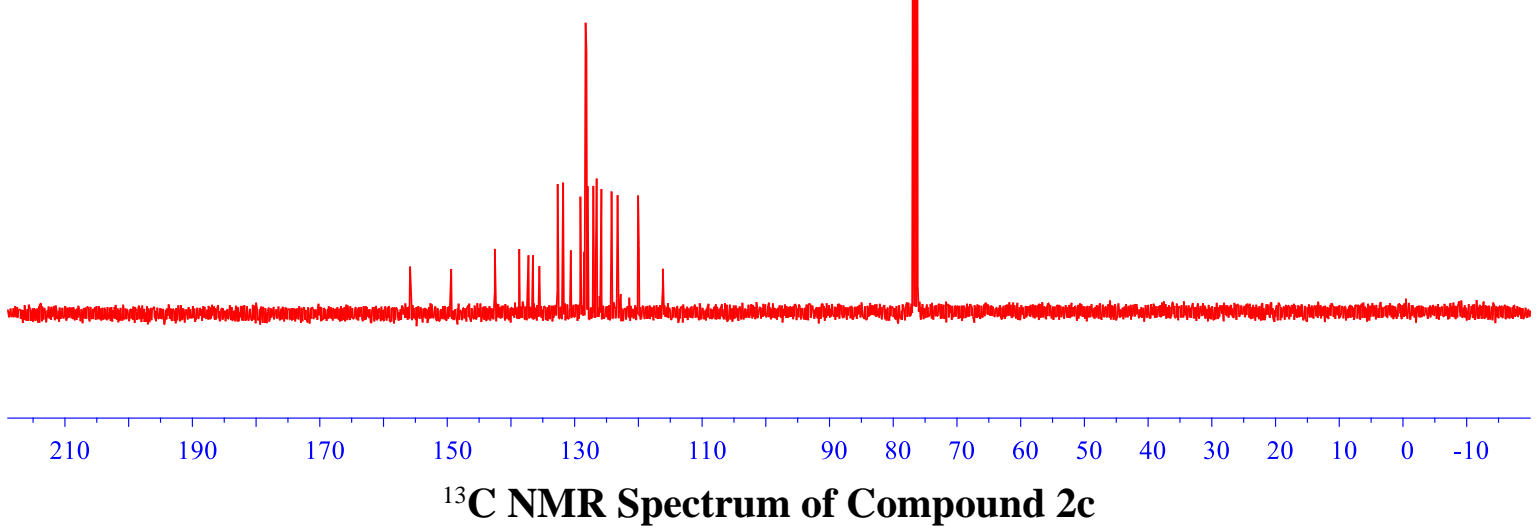




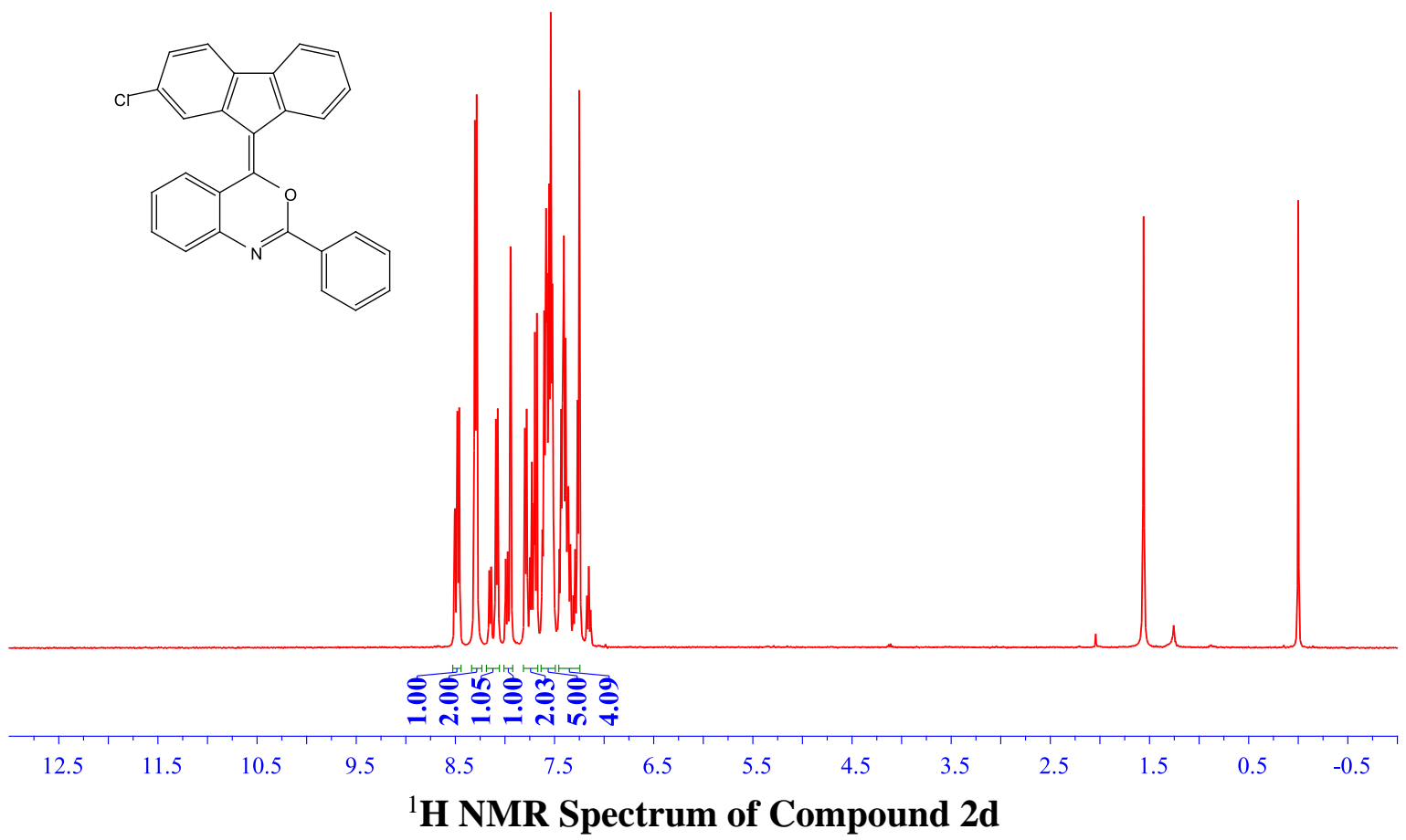

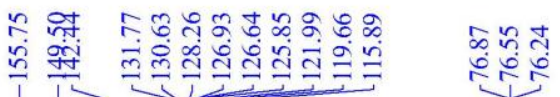
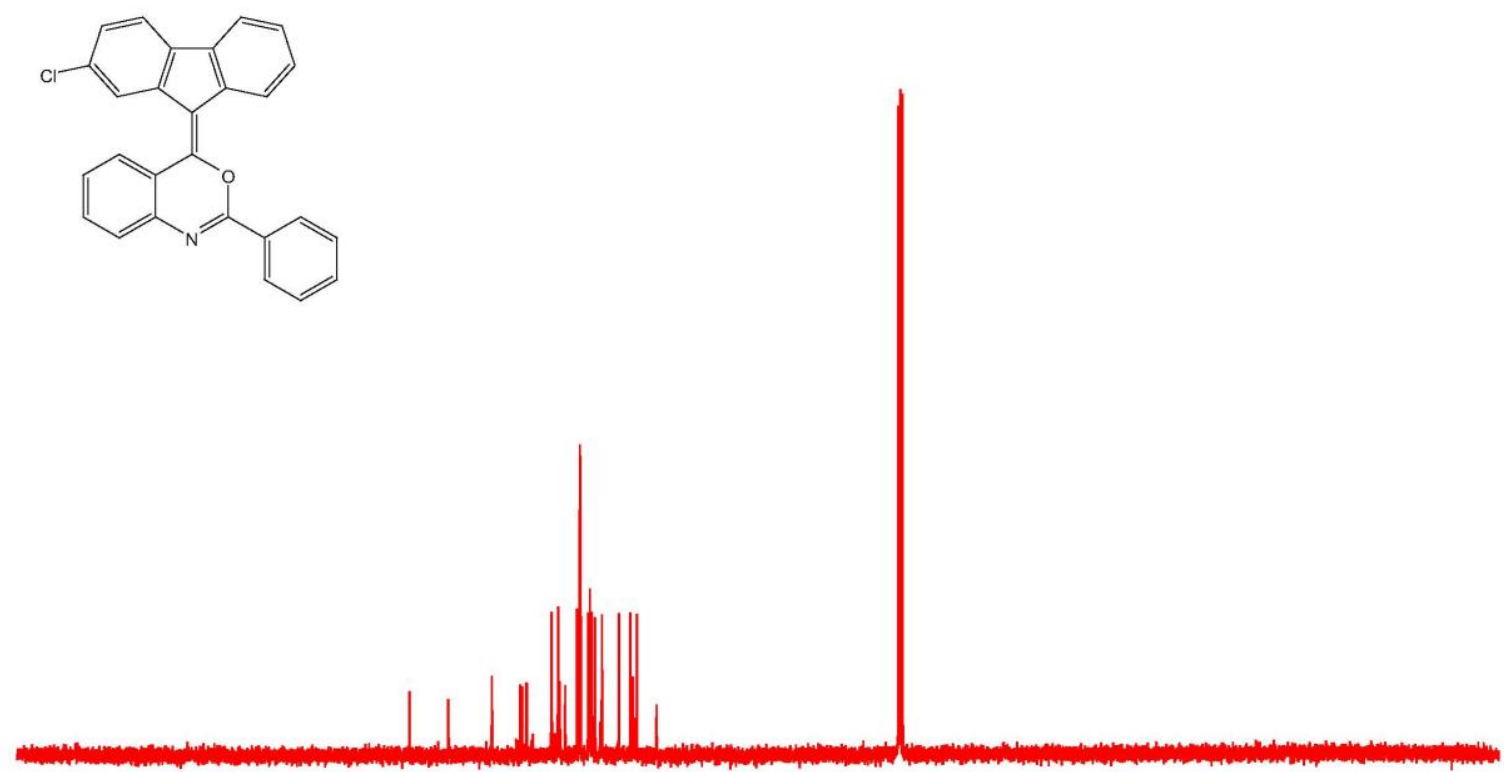

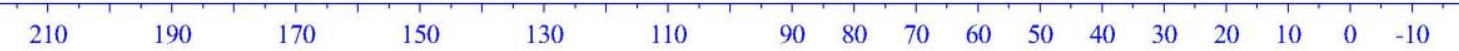

${ }^{13}$ C NMR Spectrum of Compound 2d 


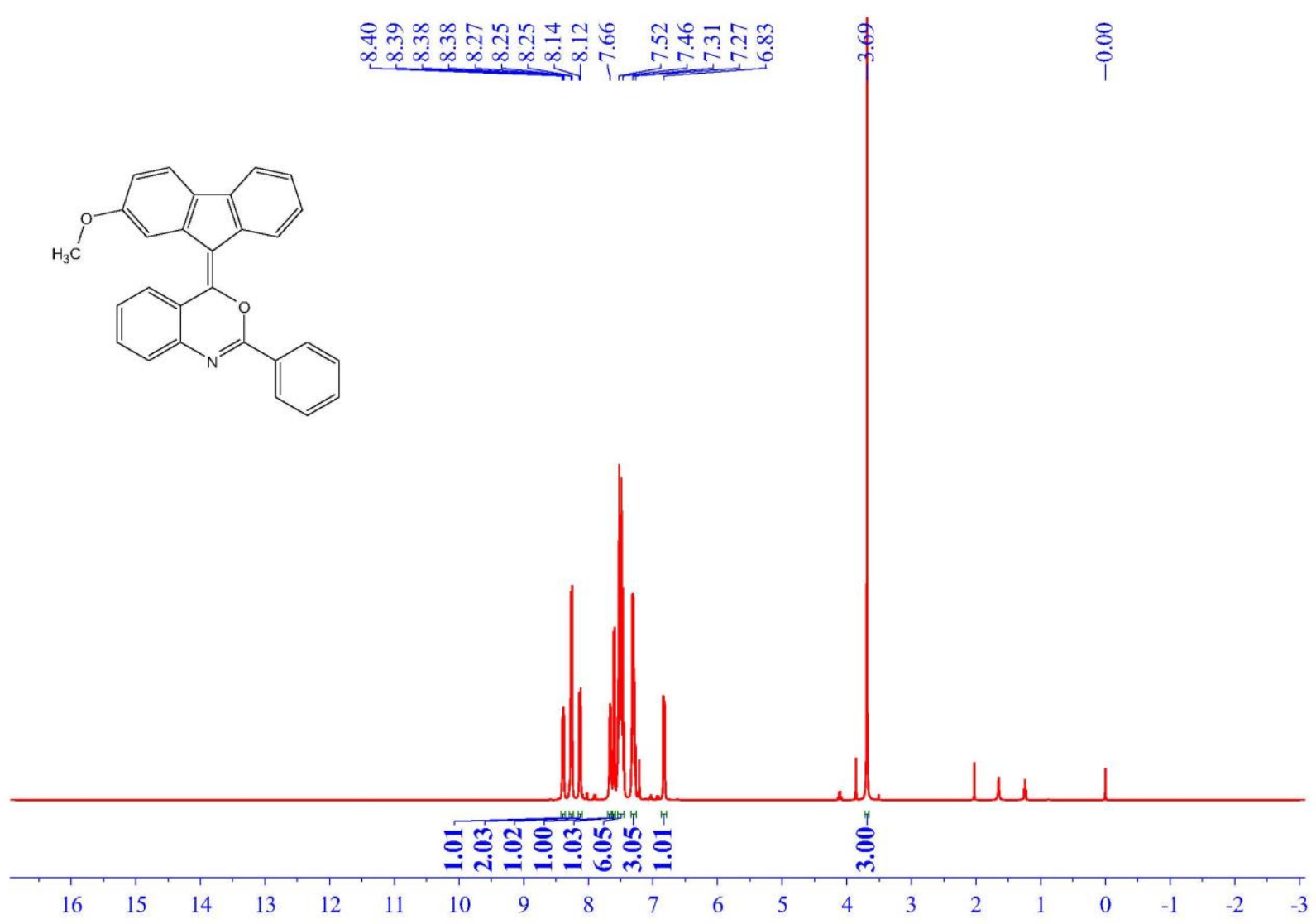

${ }^{1} \mathrm{H}$ NMR Spectrum of Compound $2 \mathrm{e}$

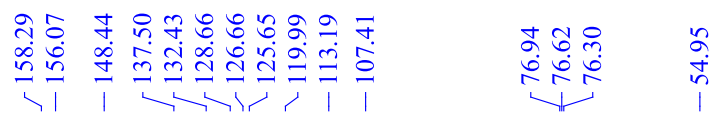<smiles>COc1ccc2c(c1)C(=C1OC(c3ccccc3)=Nc3ccccc31)c1ccccc1-2</smiles>

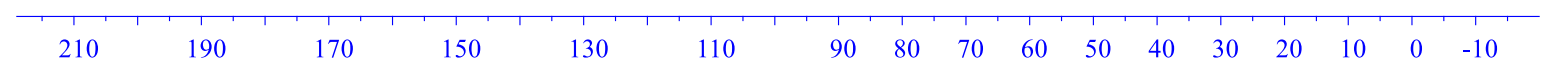


${ }^{13}$ C NMR Spectrum of Compound 2e

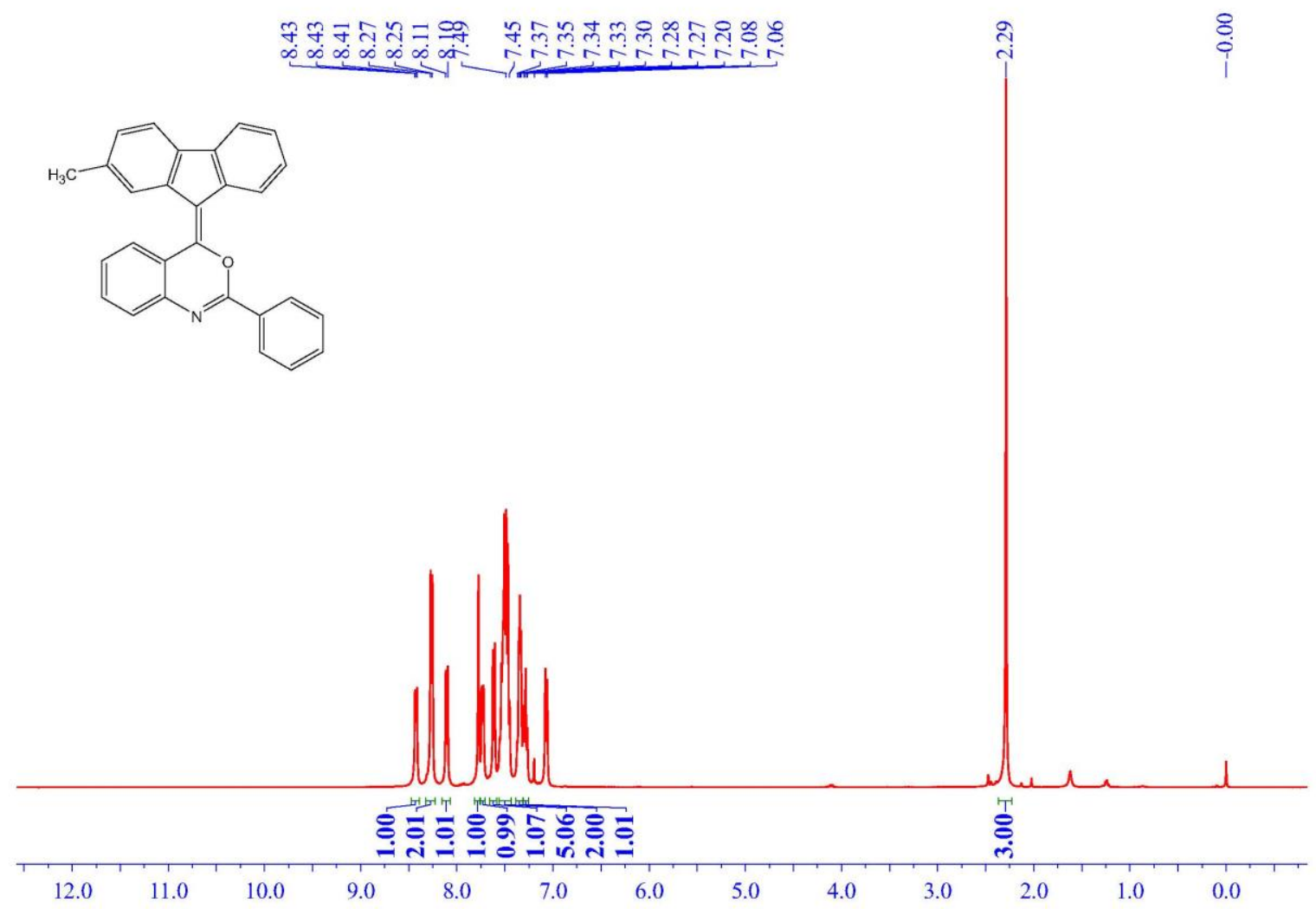

${ }^{1}$ H NMR Spectrum of Compound $2 f$

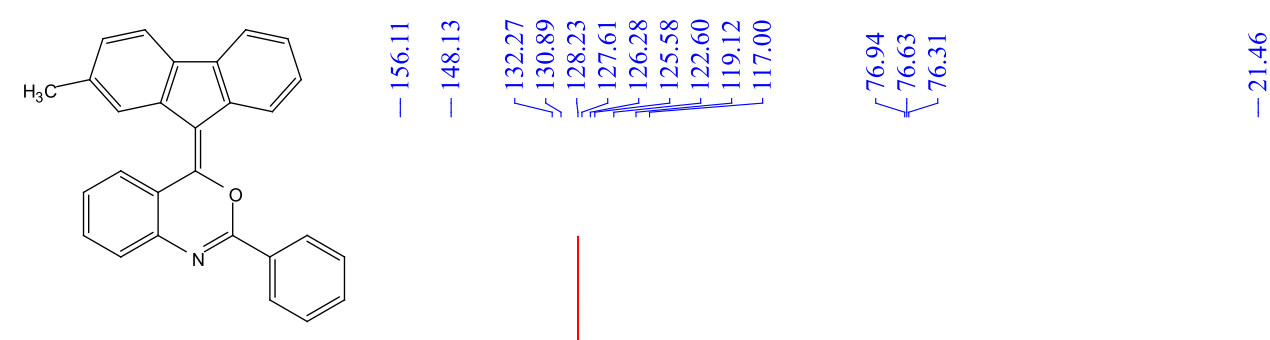

\begin{tabular}{lllllllllllllllllll}
\hline 210 & 190 & 170 & 150 & 130 & 110 & 90 & 80 & 70 & 60 & 50 & 40 & 30 & 20 & 10 & 0 & -10
\end{tabular}


${ }^{13} \mathrm{C}$ NMR Spectrum of Compound $2 f$

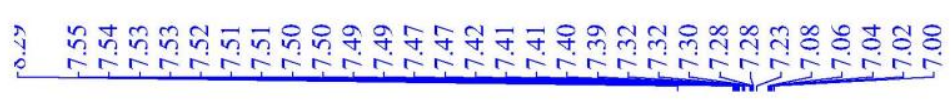

$\stackrel{8}{8}$
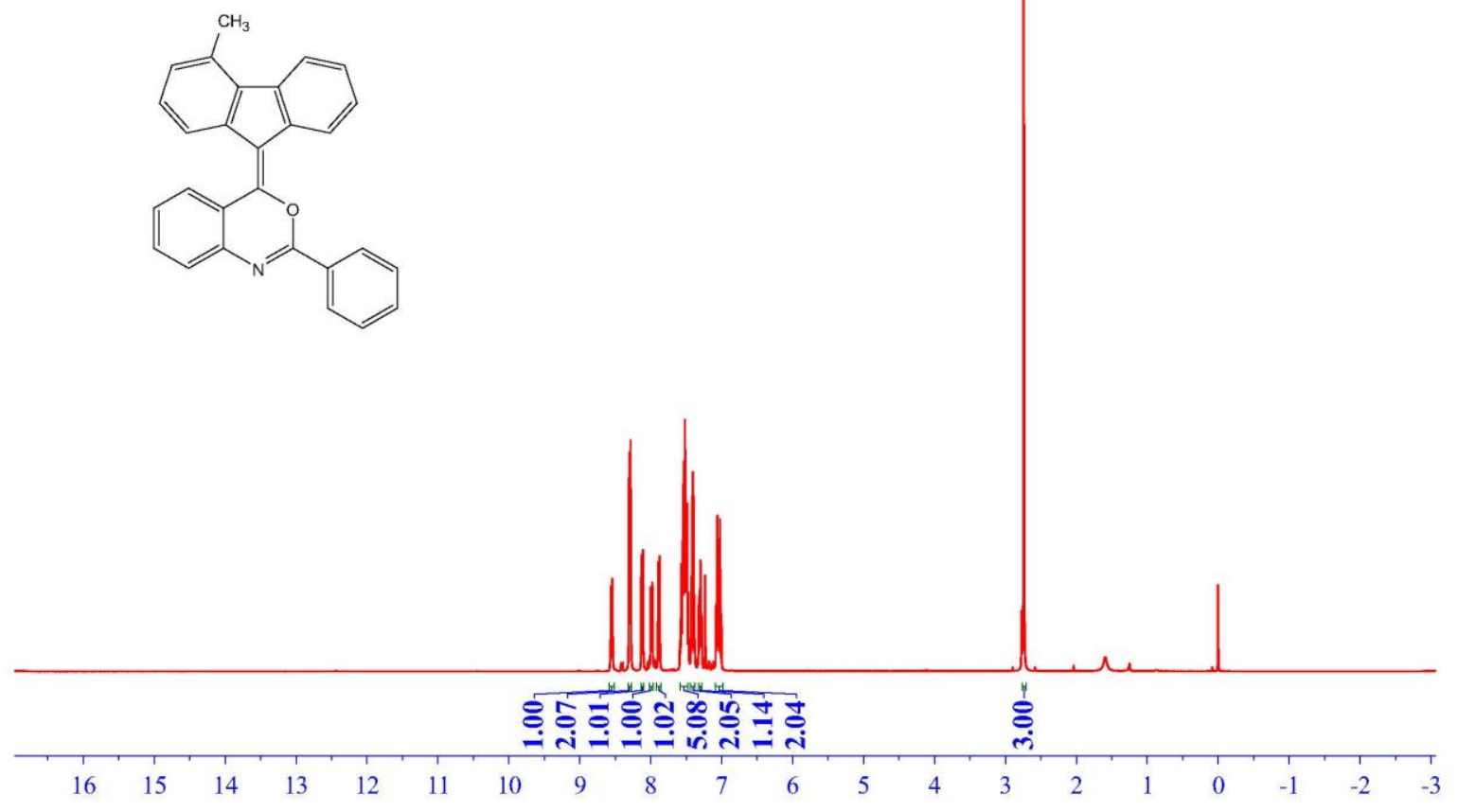

${ }^{1} \mathrm{H}$ NMR Spectrum of Compound $2 \mathrm{~g}$

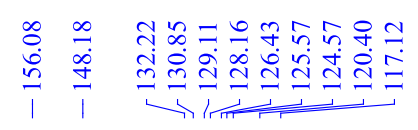

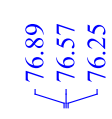

$\stackrel{\circ}{\stackrel{2}{2}}$
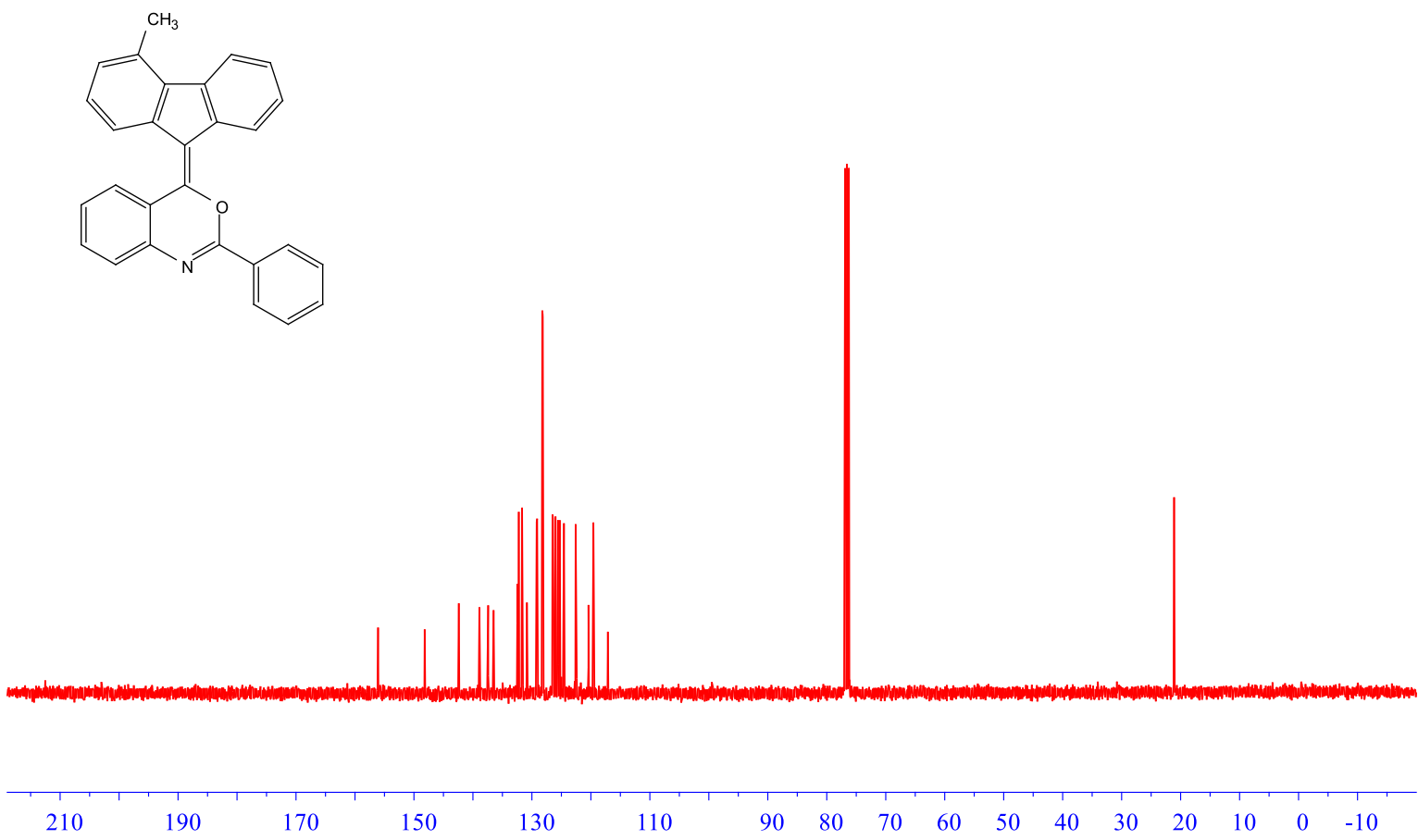

S18 
${ }^{13}$ C NMR Spectrum of Compound $2 \mathrm{~g}$

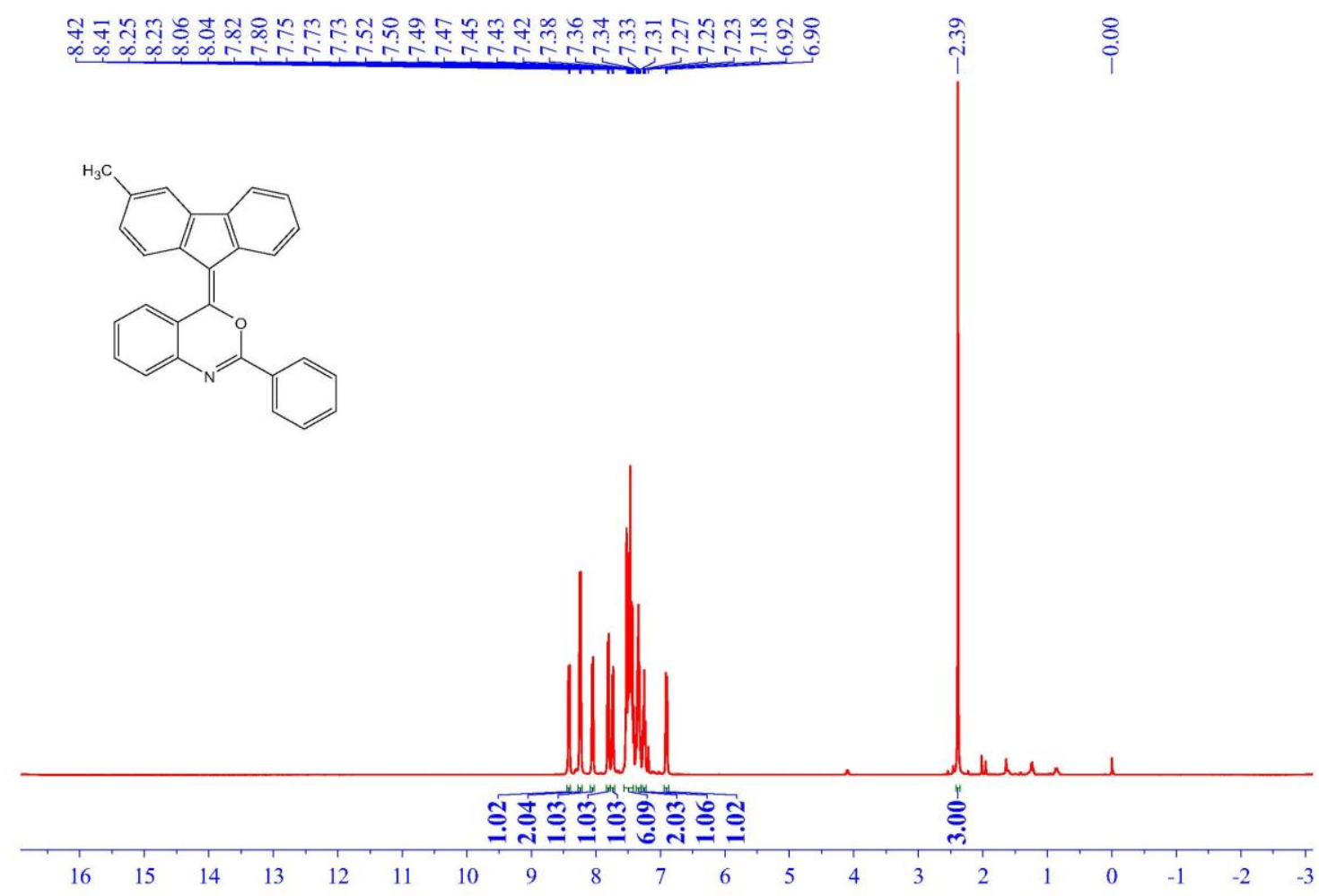

${ }^{1} \mathrm{H}$ NMR Spectrum of Compound $2 \mathrm{~h}$

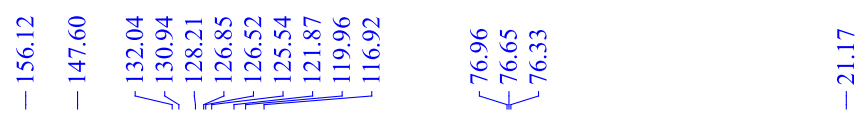
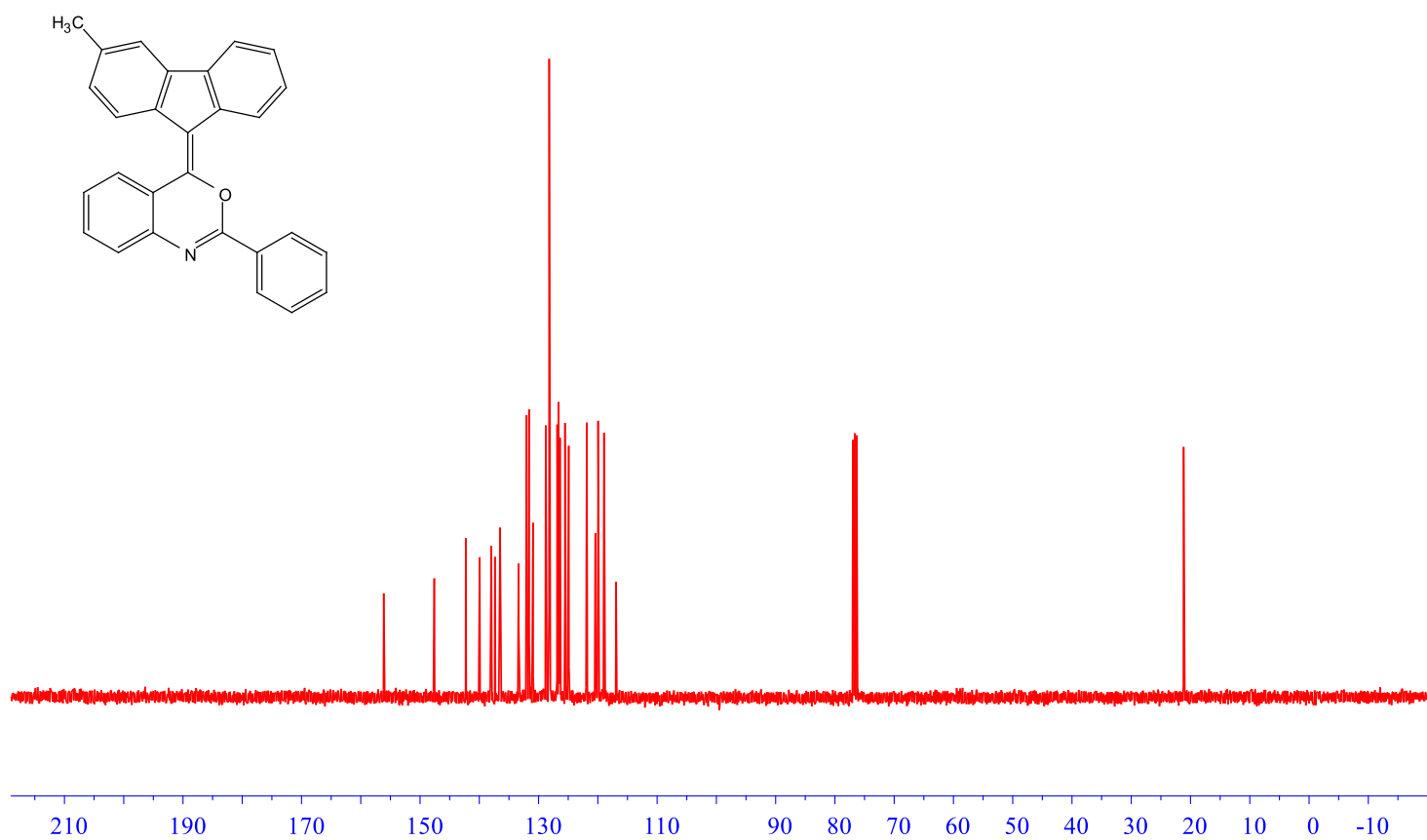

${ }^{13} \mathrm{C}$ NMR Spectrum of Compound $2 \mathrm{~h}$ 

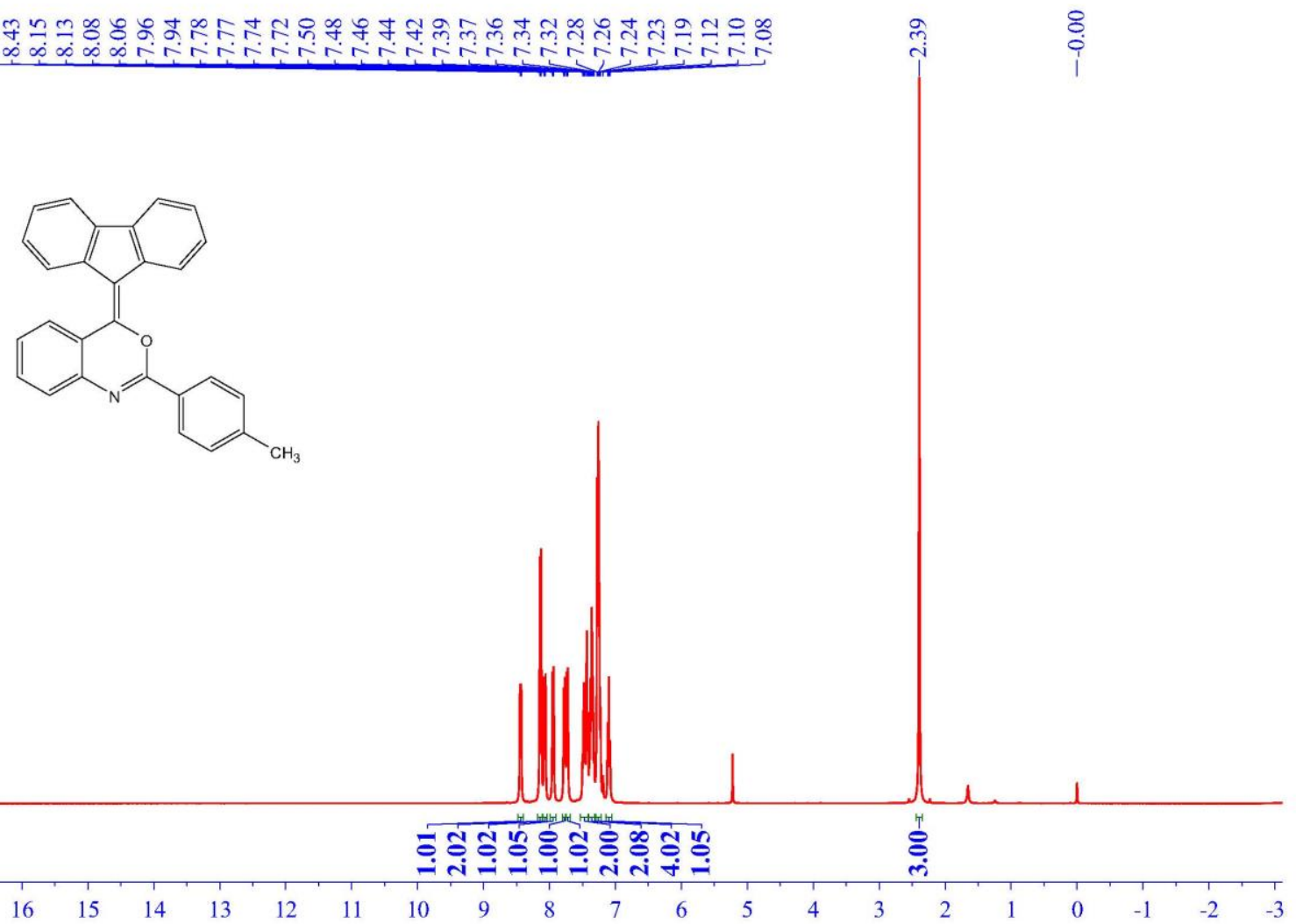

${ }^{1} \mathrm{H}$ NMR Spectrum of Compound $2 \mathrm{i}$
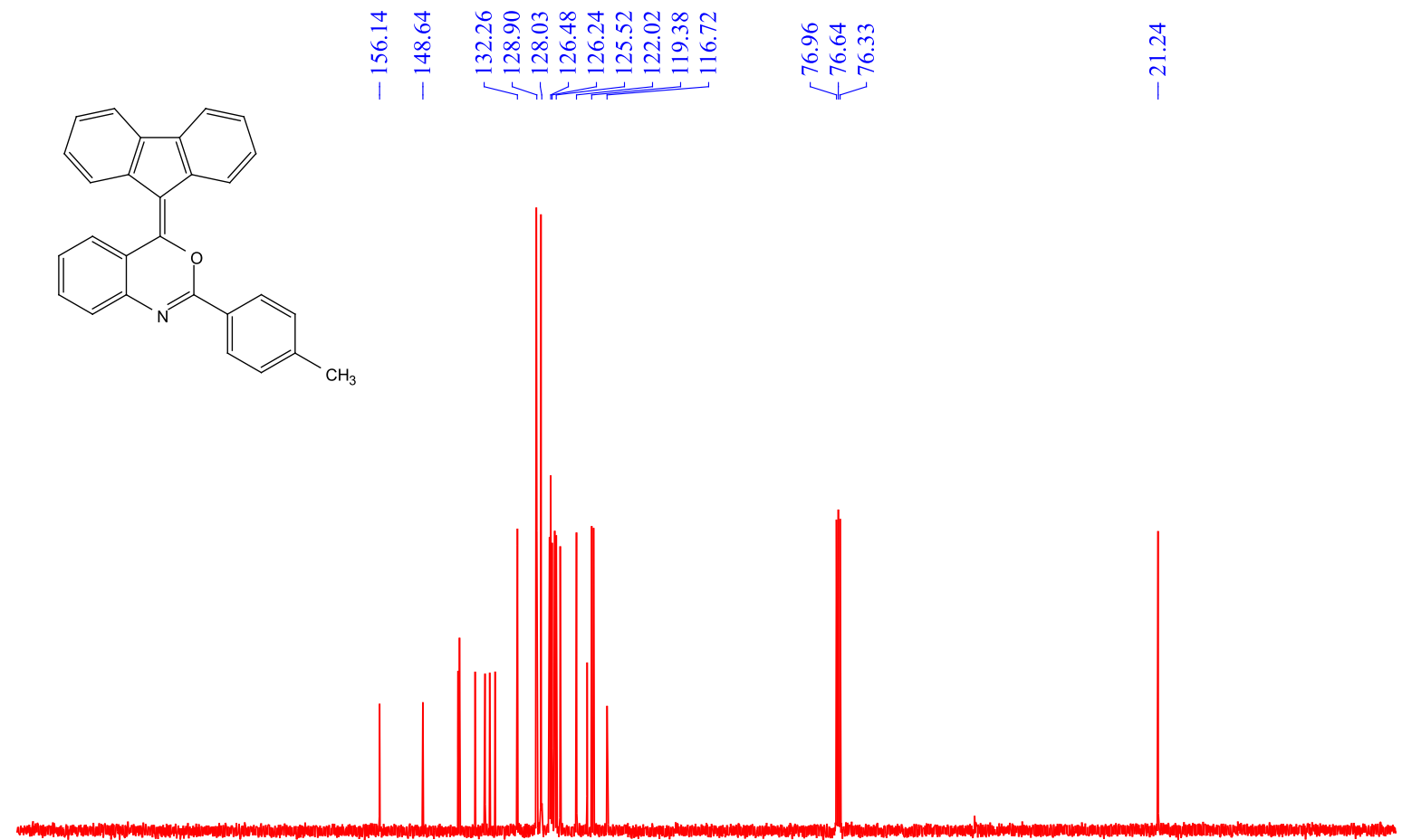

${ }^{13} \mathrm{C}$ NMR Spectrum of Compound $2 \mathrm{i}$ 


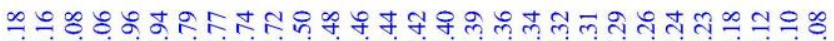

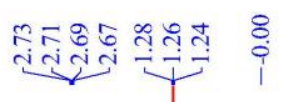
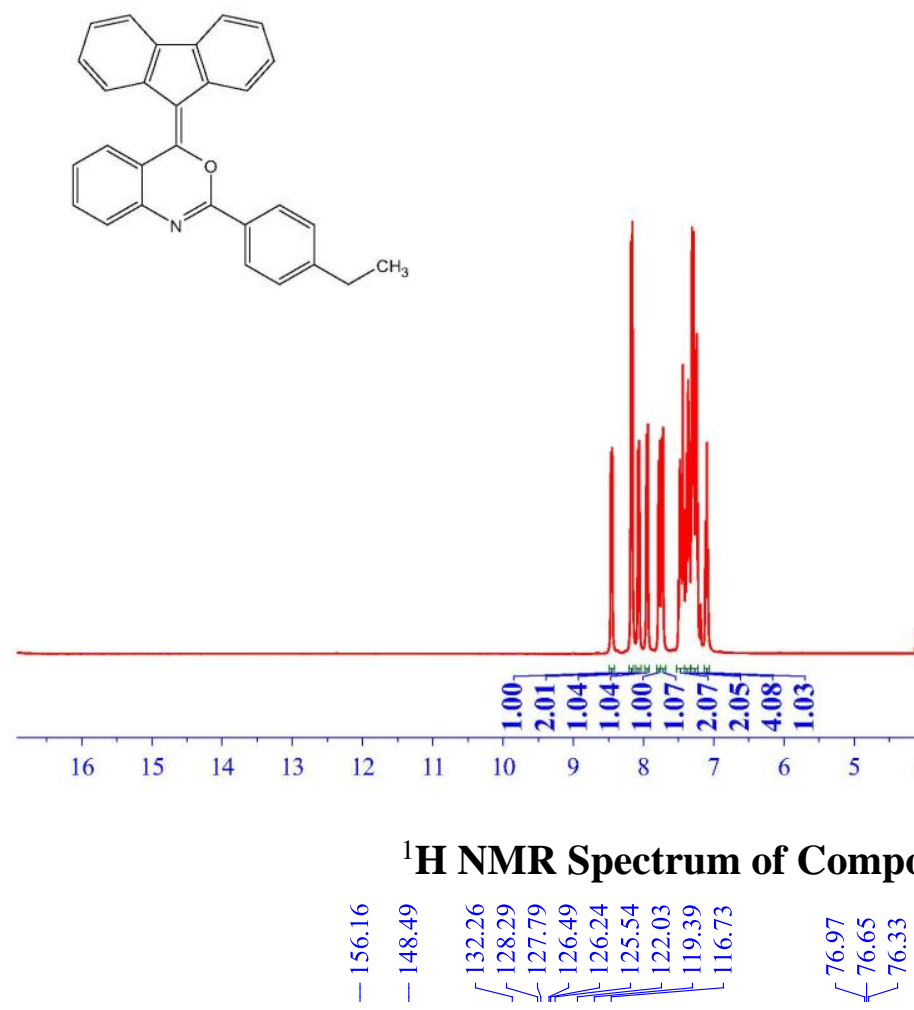

$\begin{array}{ll}\sqrt{n} & \vec{\infty} \\ \substack{\infty \\ 0} & 1\end{array}$
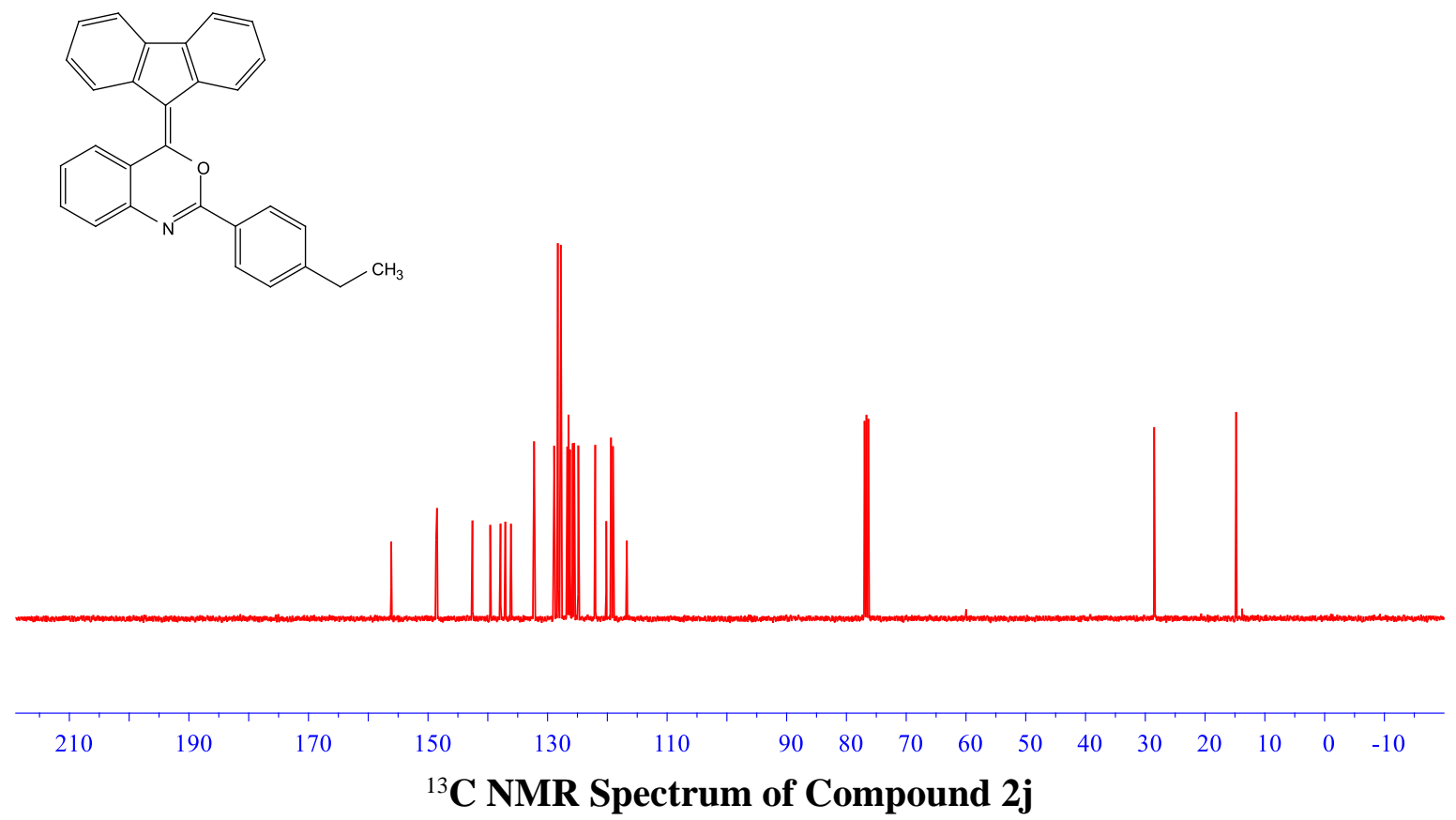

S21 


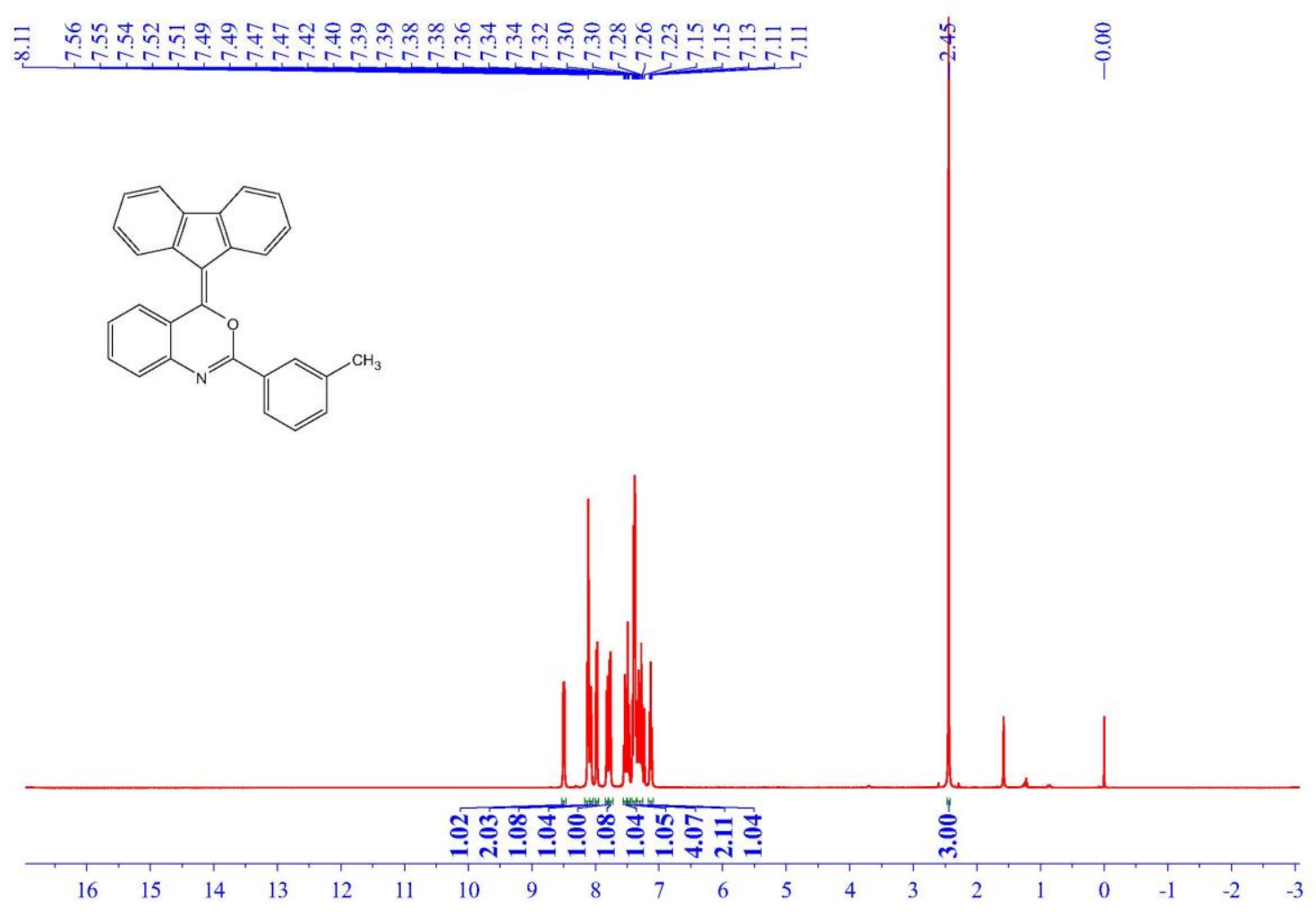

${ }^{1}$ H NMR Spectrum of Compound 2k
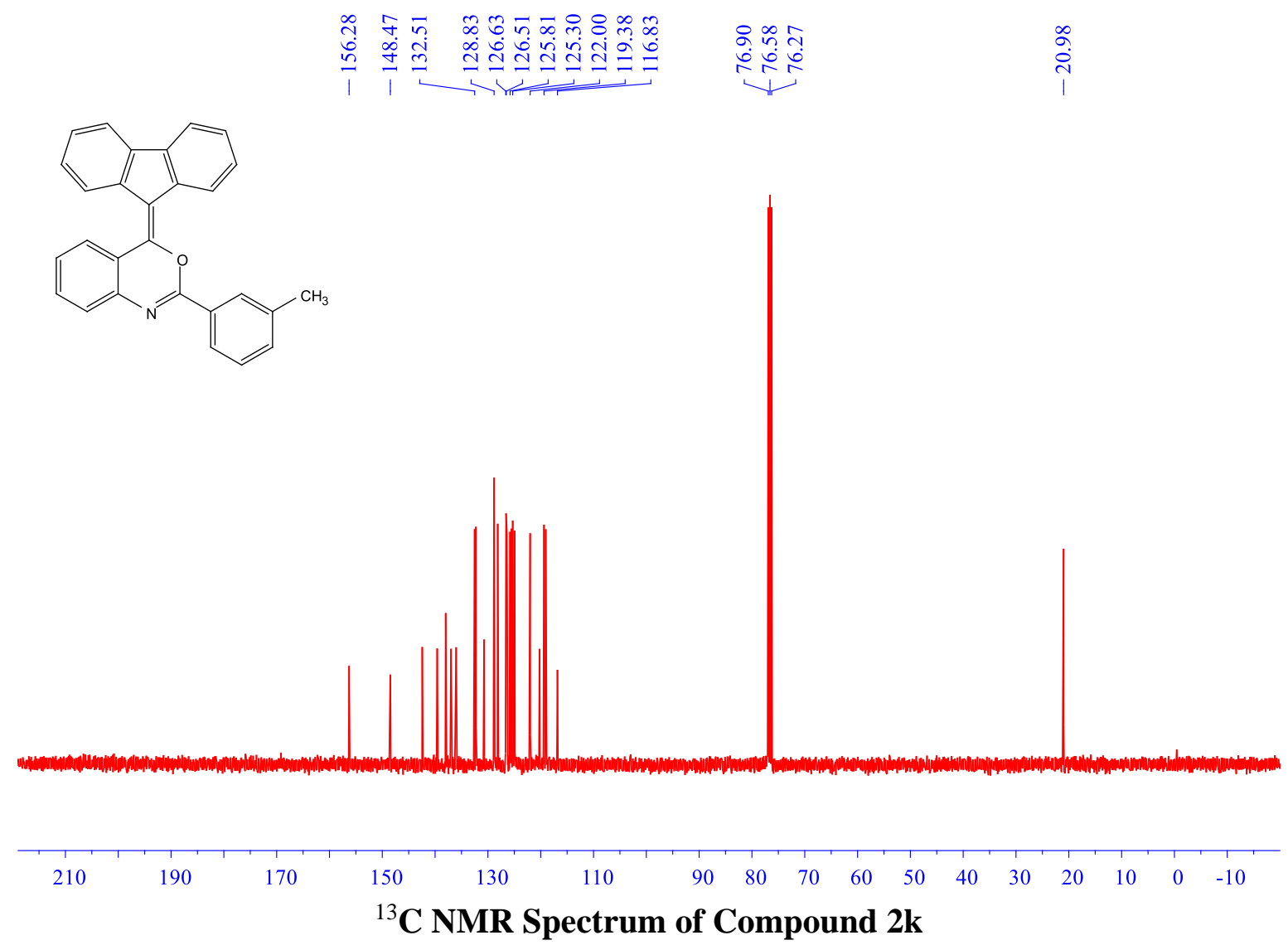

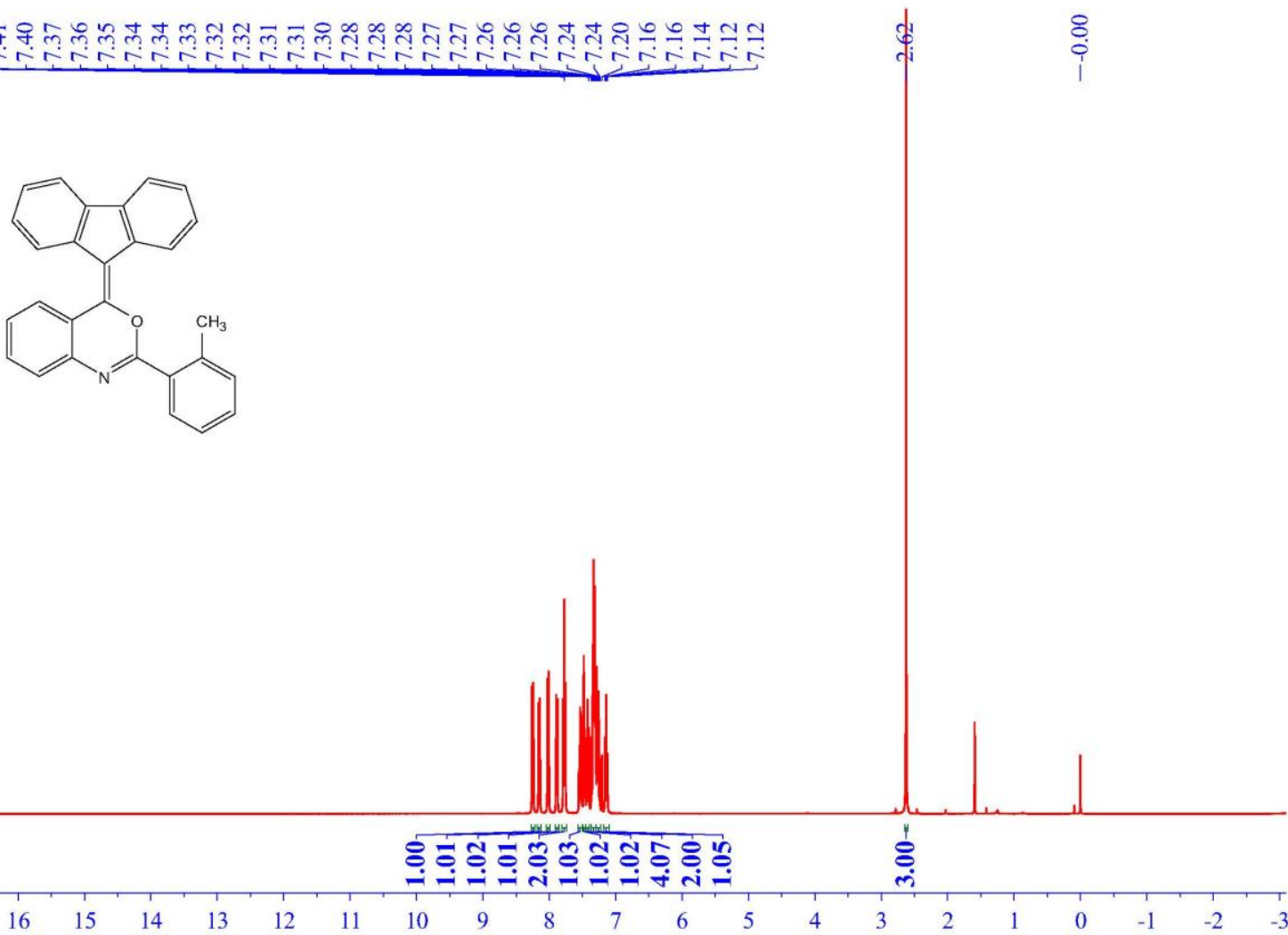

${ }^{1}$ H NMR Spectrum of Compound 21

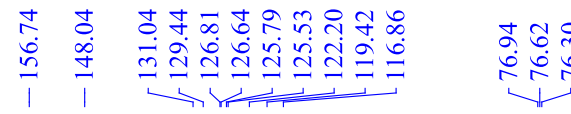<smiles>Cc1ccccc1C1=Nc2ccccc2C(=C2c3ccccc3-c3ccccc32)O1</smiles>

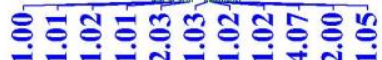

$\begin{array}{llllllllllllllllllll}16 & 15 & 14 & 13 & 12 & 11 & 10 & 9 & 8 & 7 & 6 & 5 & 4 & 3 & 2 & 1 & 0 & -1 & -2 & -3\end{array}$




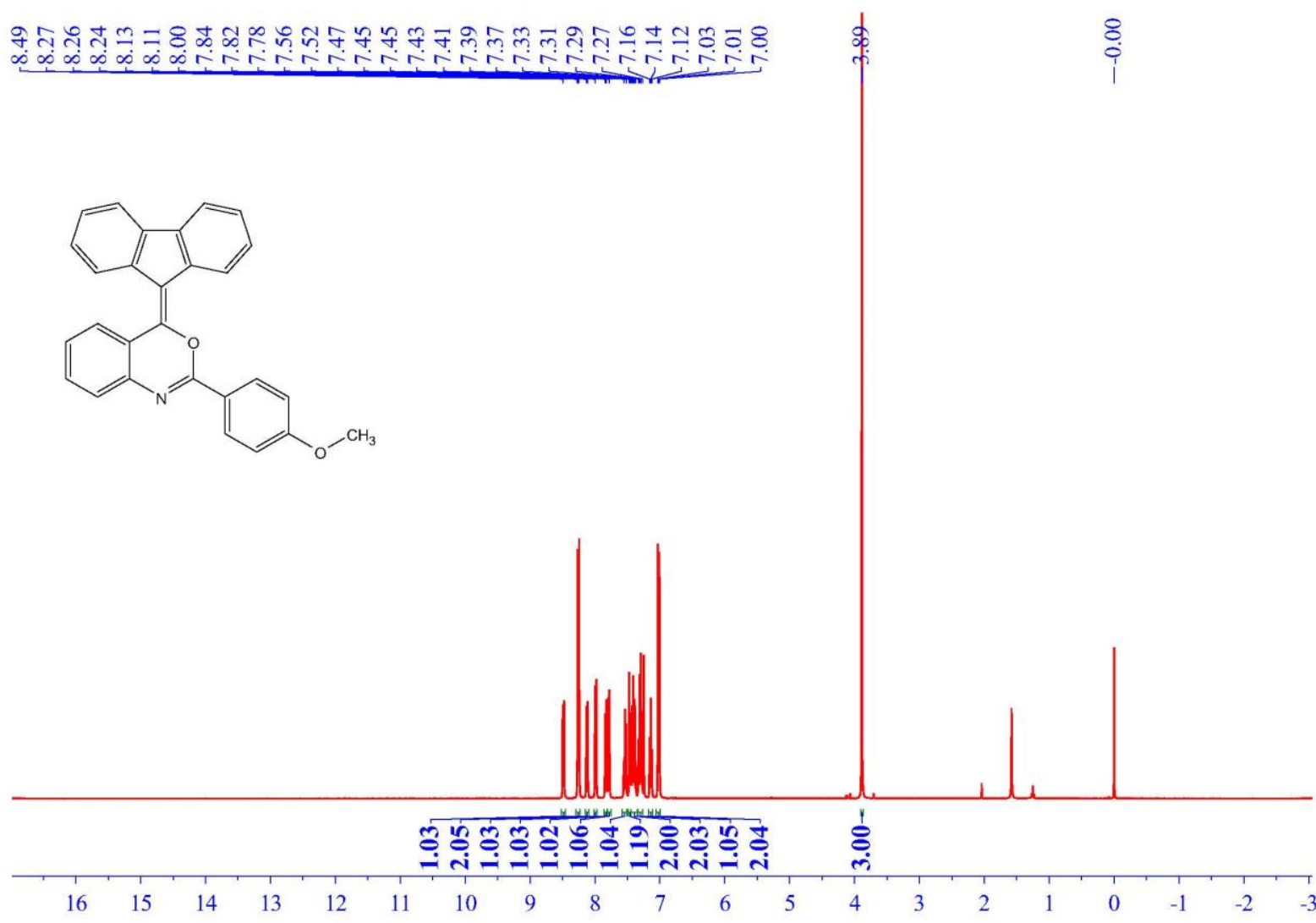

${ }^{1}$ H NMR Spectrum of Compound $2 \mathrm{~m}$

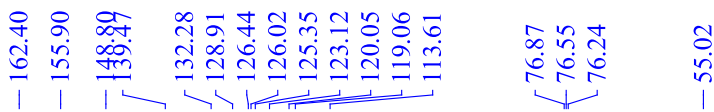
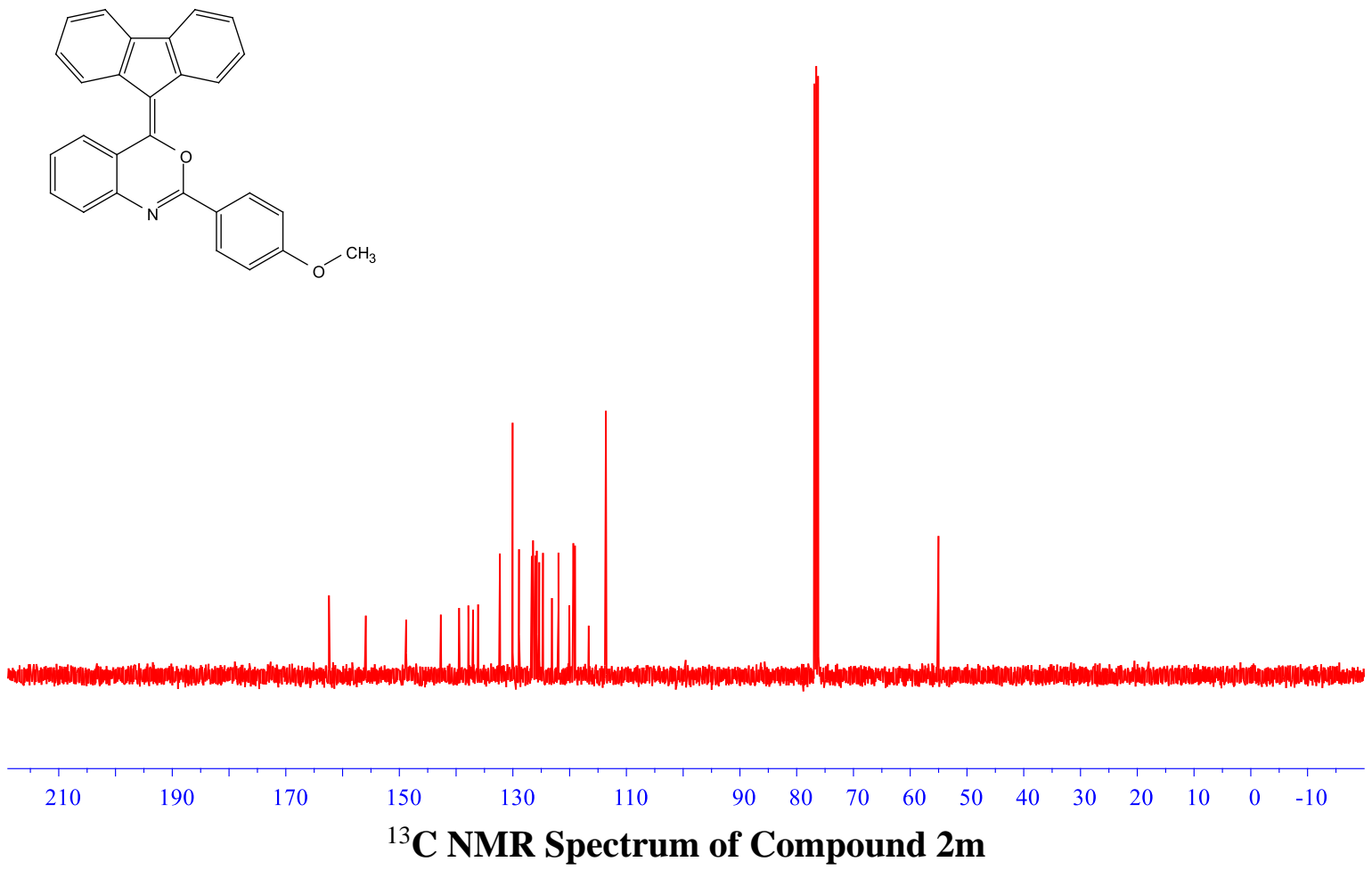


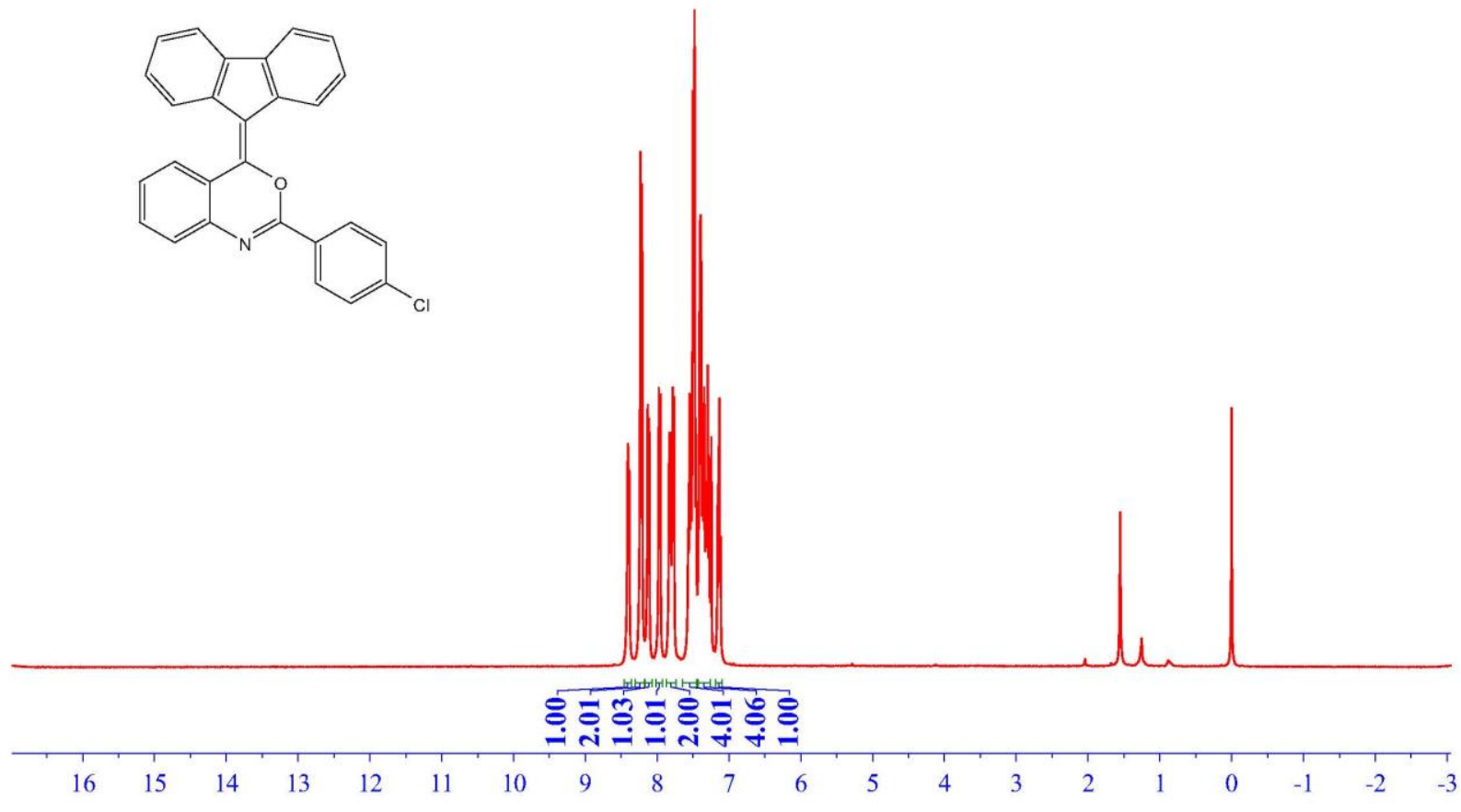

${ }^{1} H$ NMR Spectrum of Compound 2n

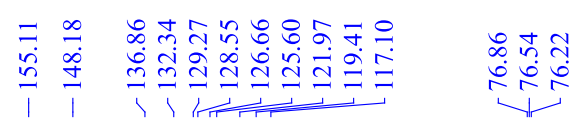
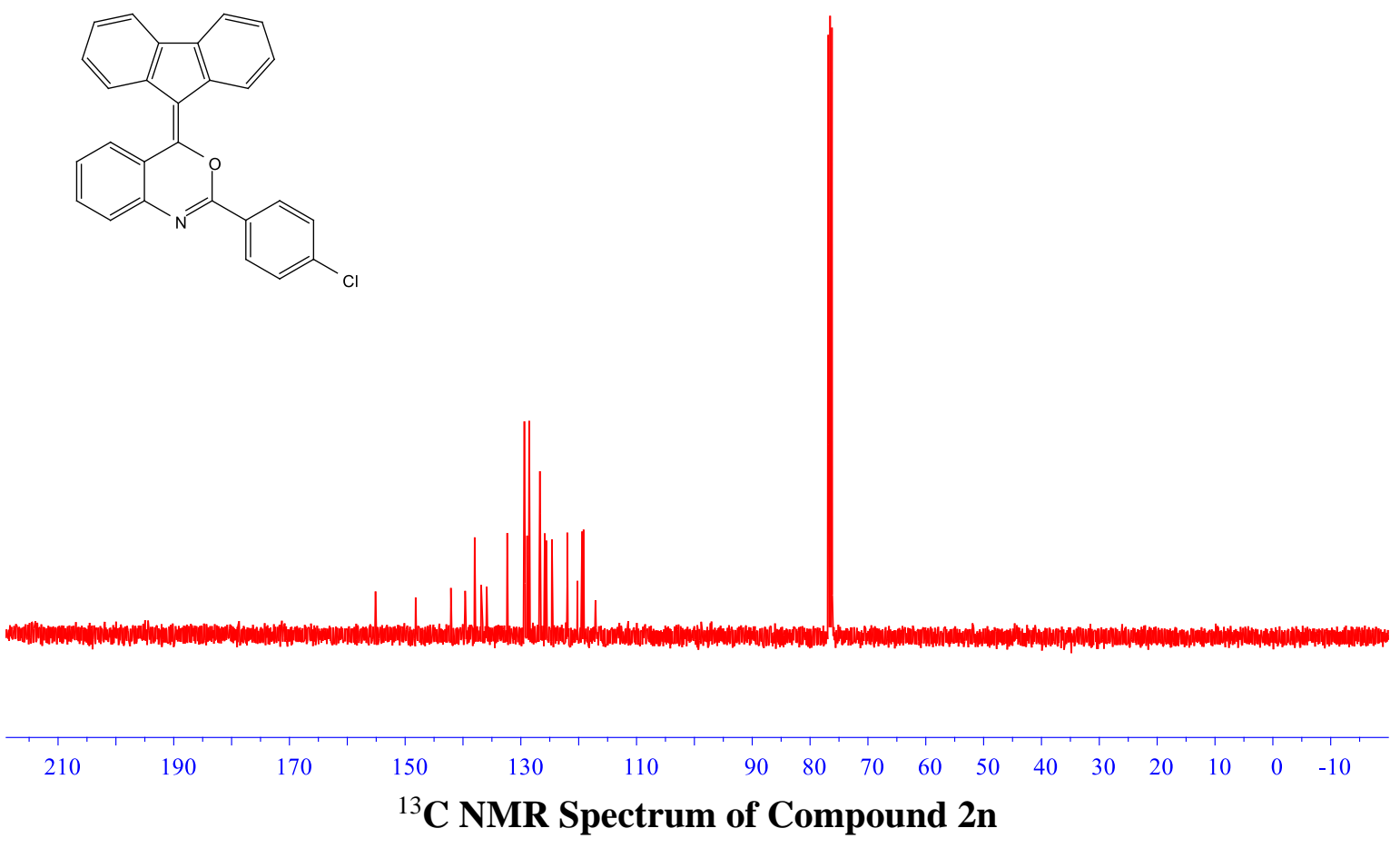


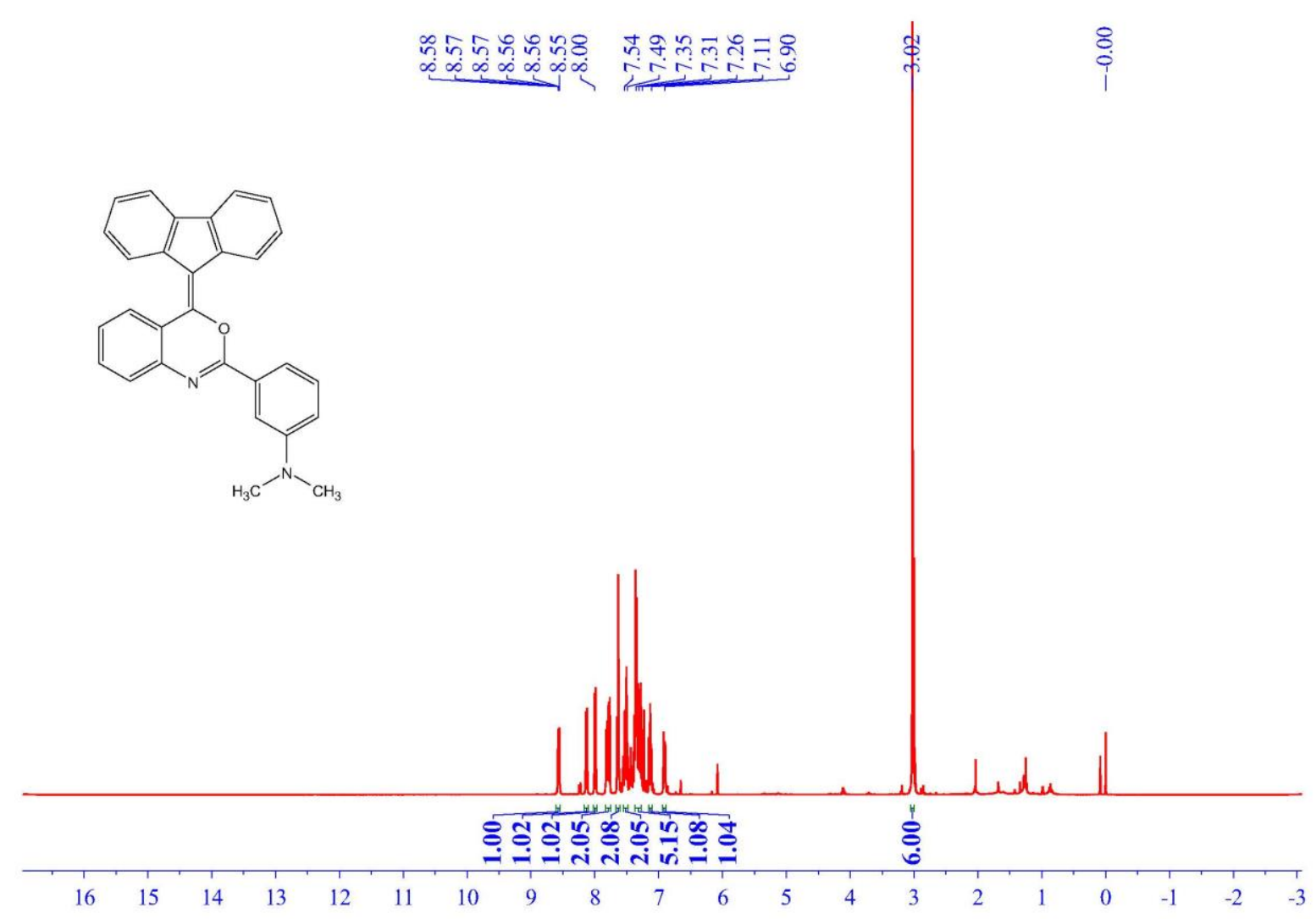

${ }^{1}$ H NMR Spectrum of Compound 20

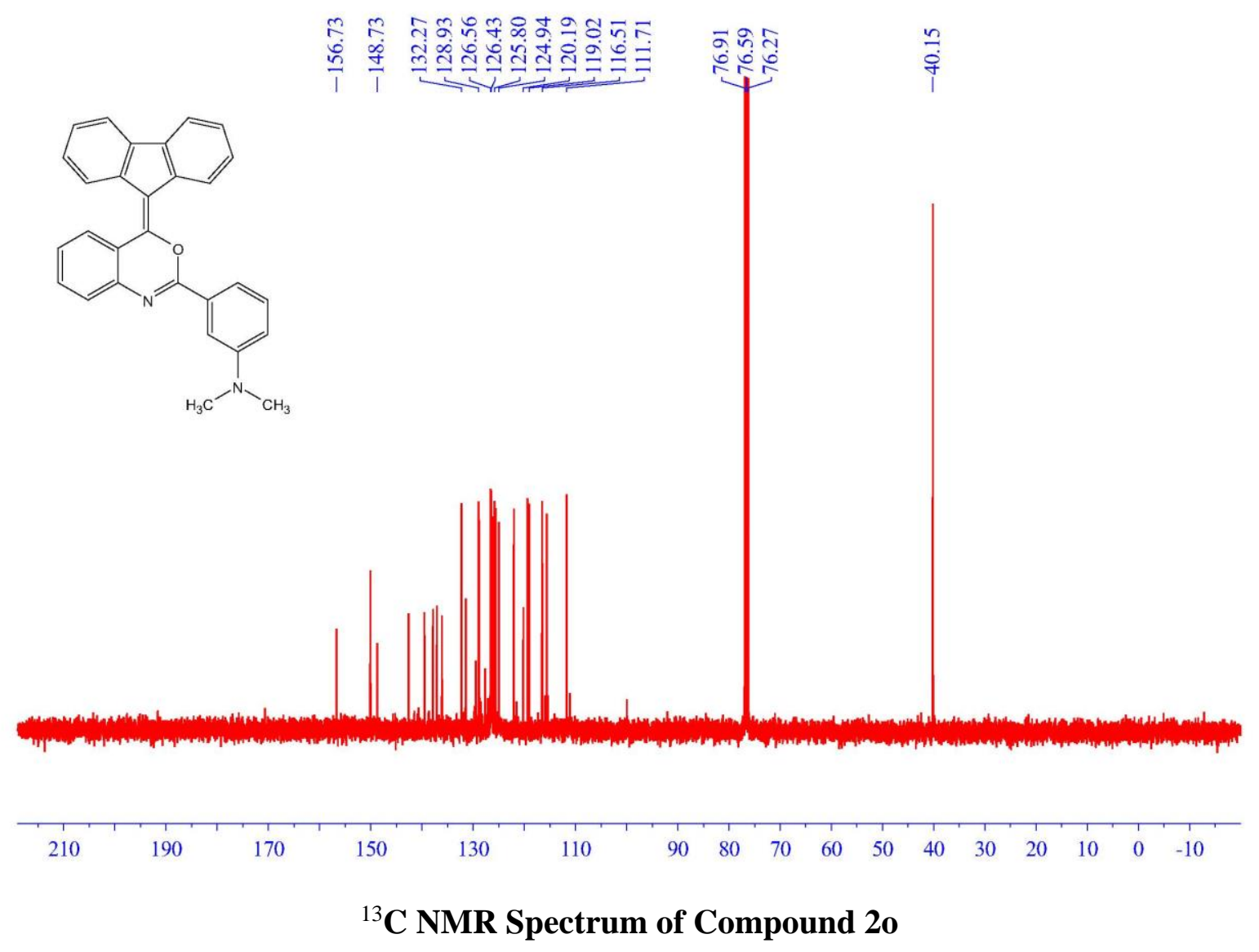




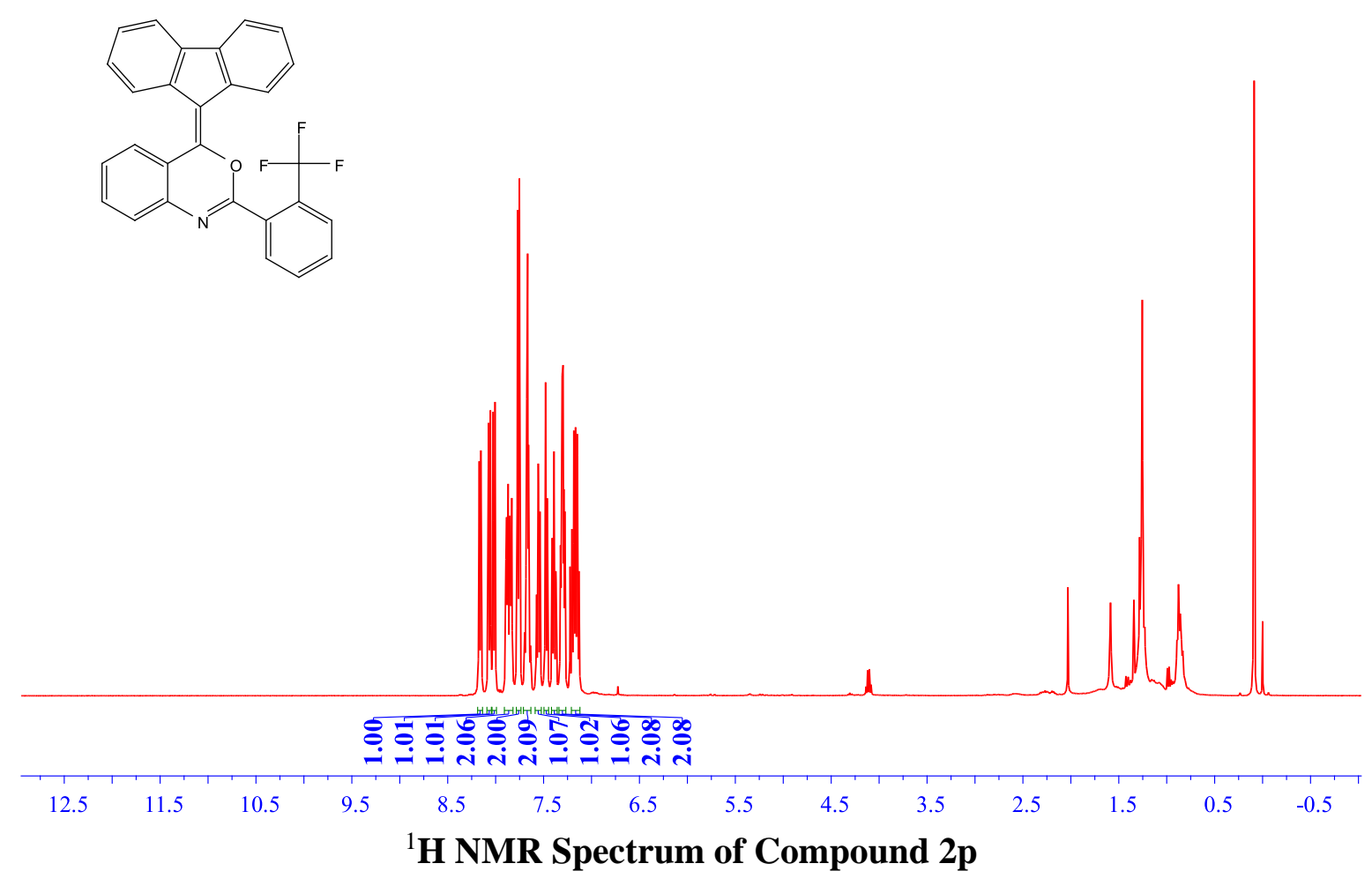

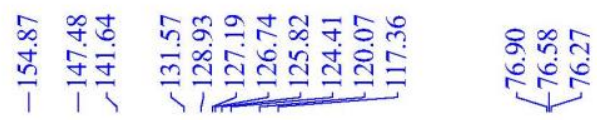
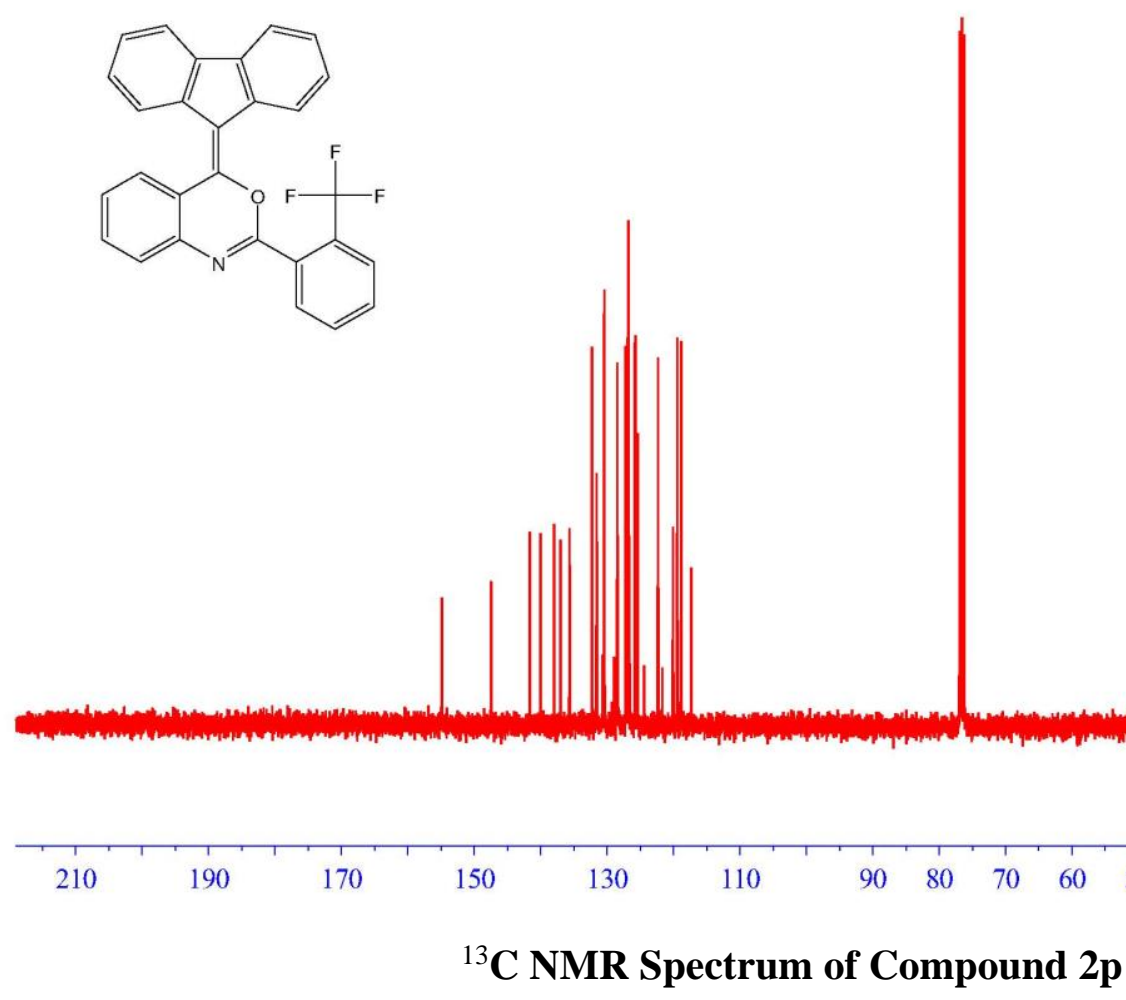


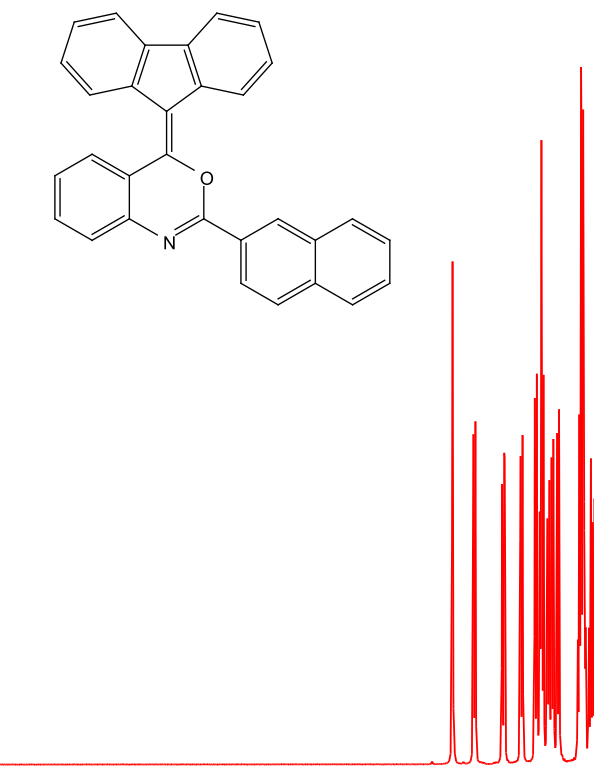

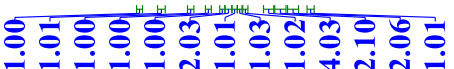

\begin{tabular}{|c|c|c|c|c|c|c|c|c|c|c|c|c|c|c|}
\hline 13.0 & 12.0 & 11.0 & 10.0 & 9.0 & 8.0 & 7.0 & 6.0 & 5.0 & 4.0 & 3.0 & 2.0 & 1.0 & 0.0 & -1.0 \\
\hline
\end{tabular}

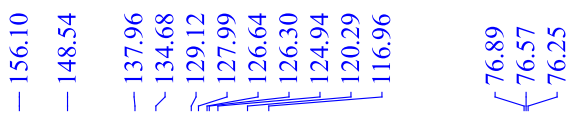
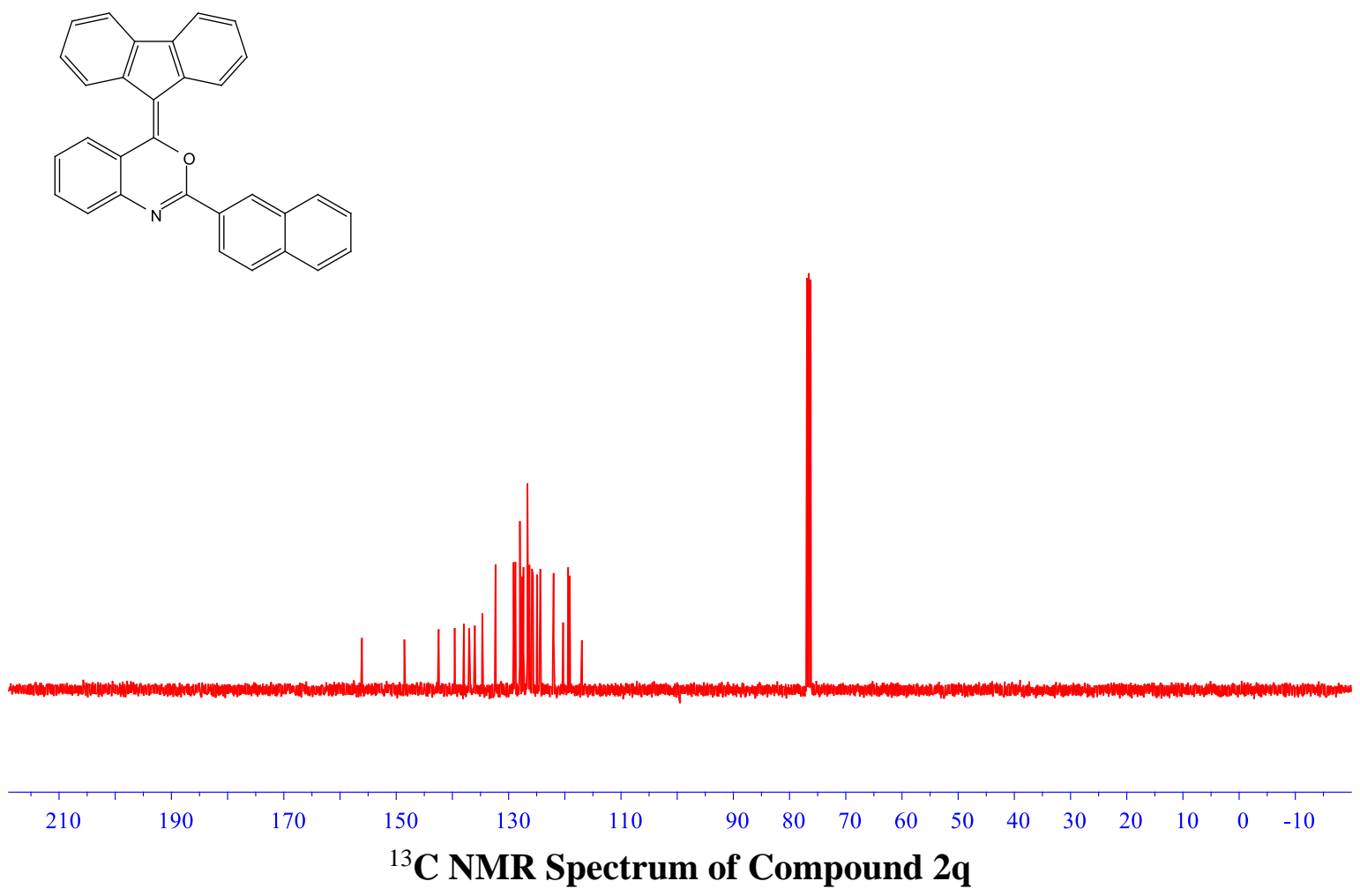

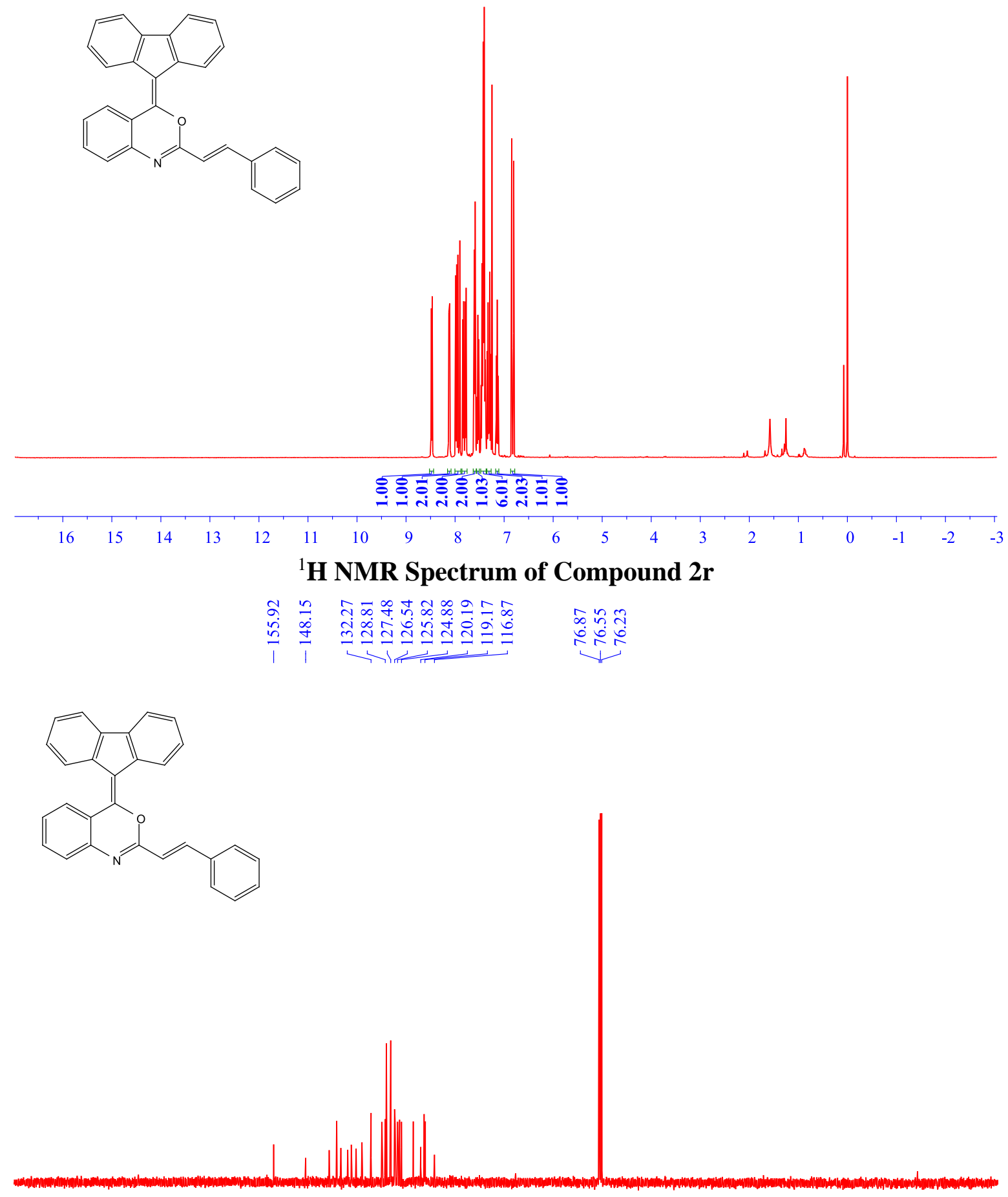

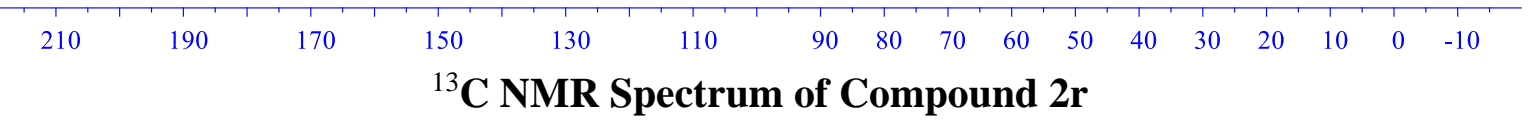



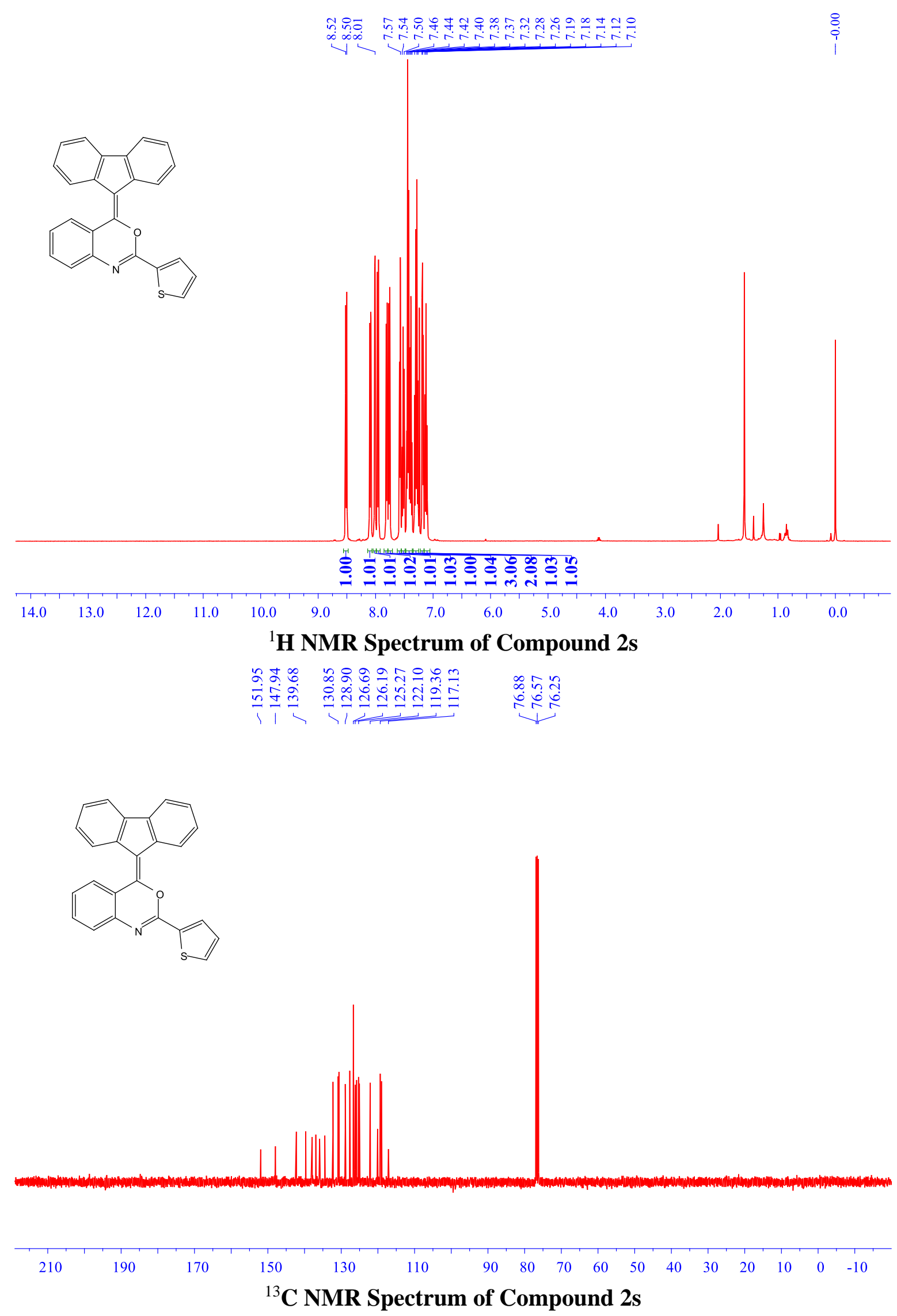

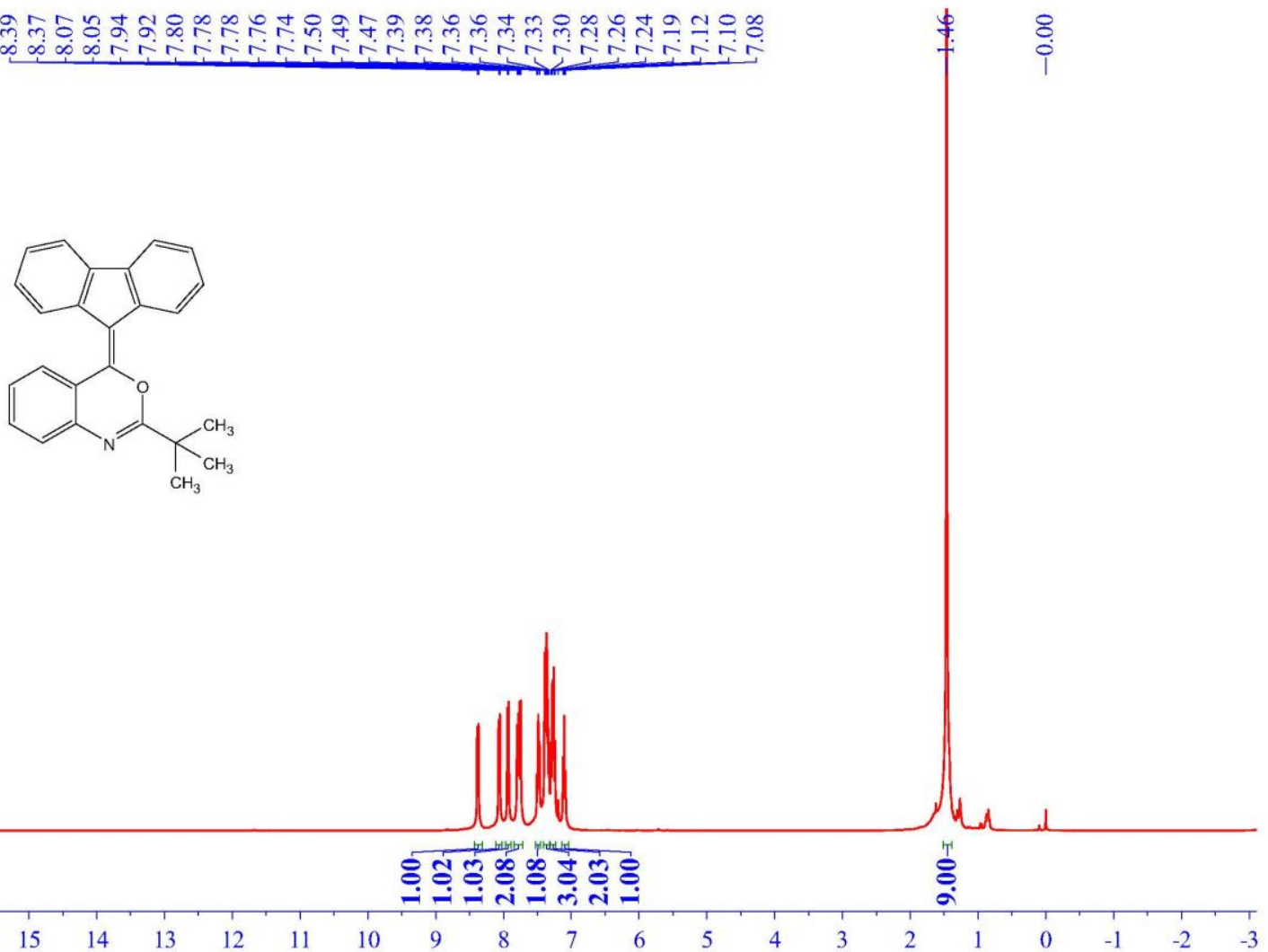

${ }^{1} \mathrm{H}$ NMR Spectrum of Compound $2 \mathrm{t}$

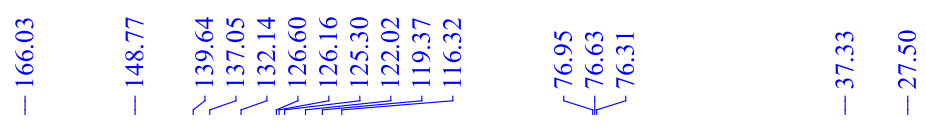
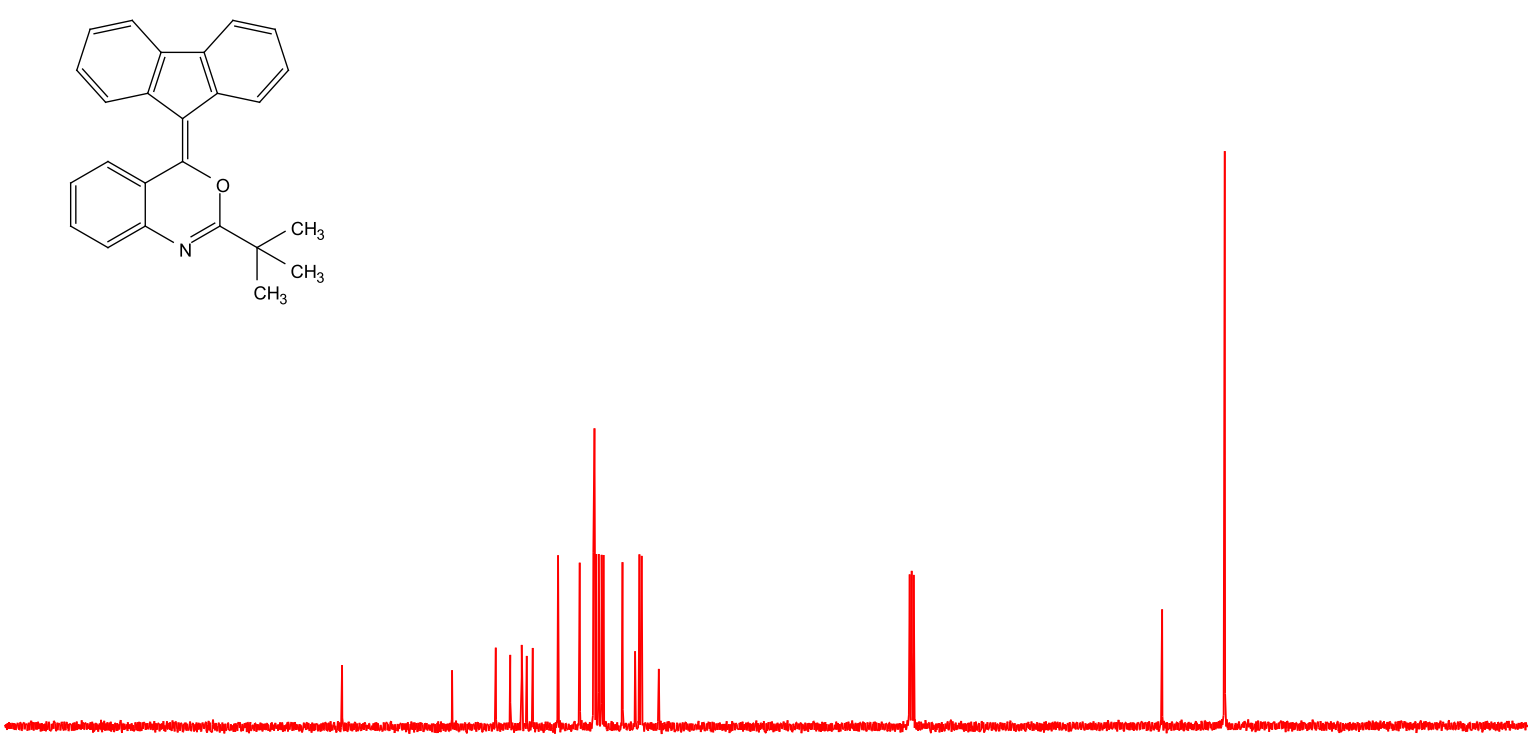

${ }^{13}$ C NMR Spectrum of Compound $2 t$ 


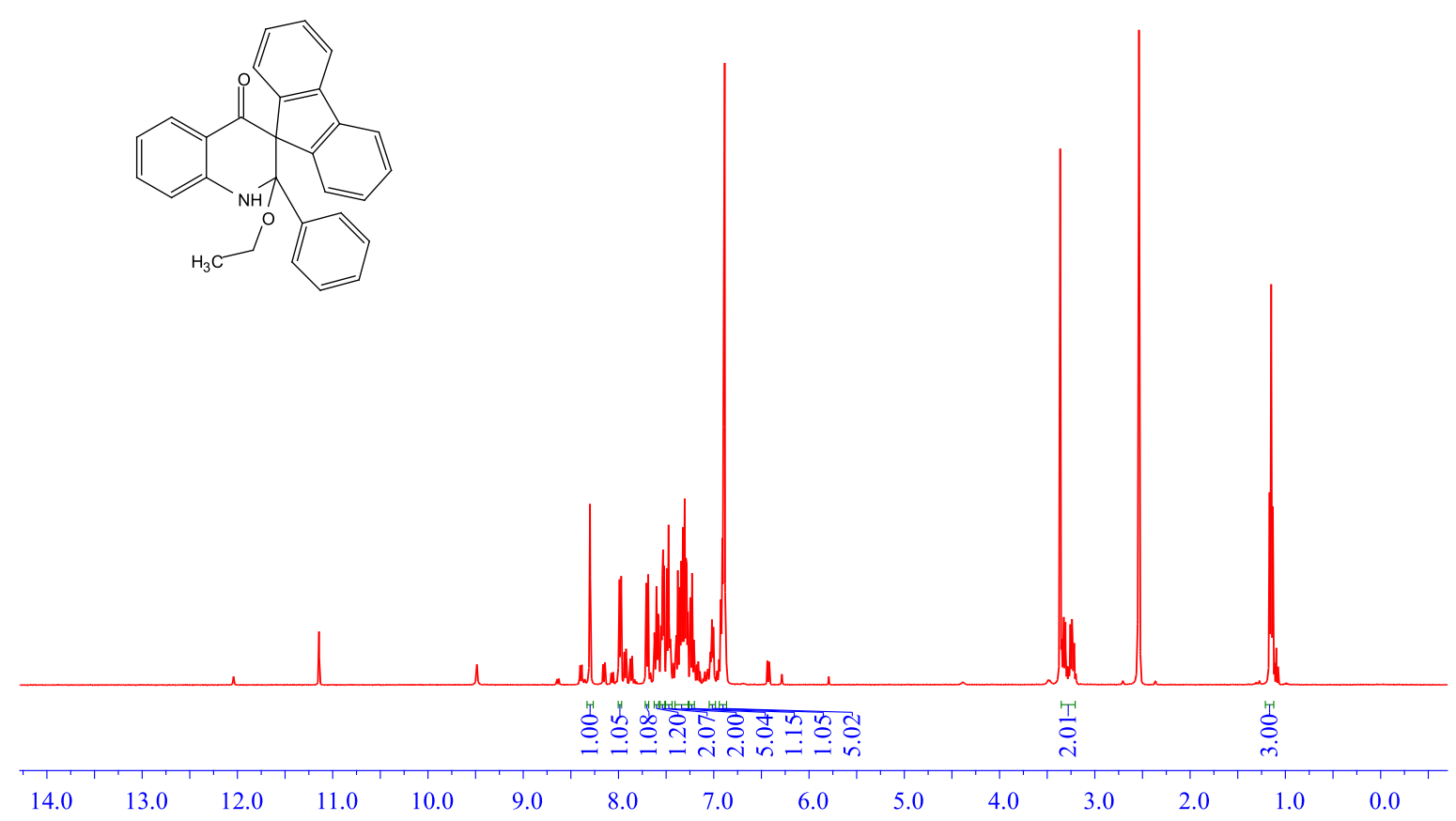

${ }^{1} H$ NMR Spectrum of Compound 2a'

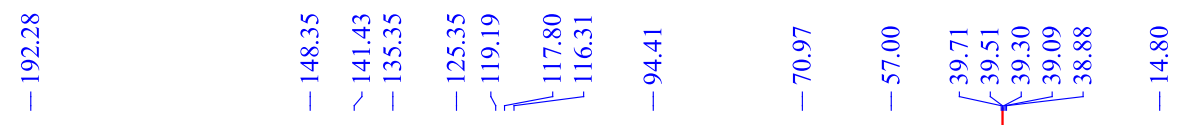
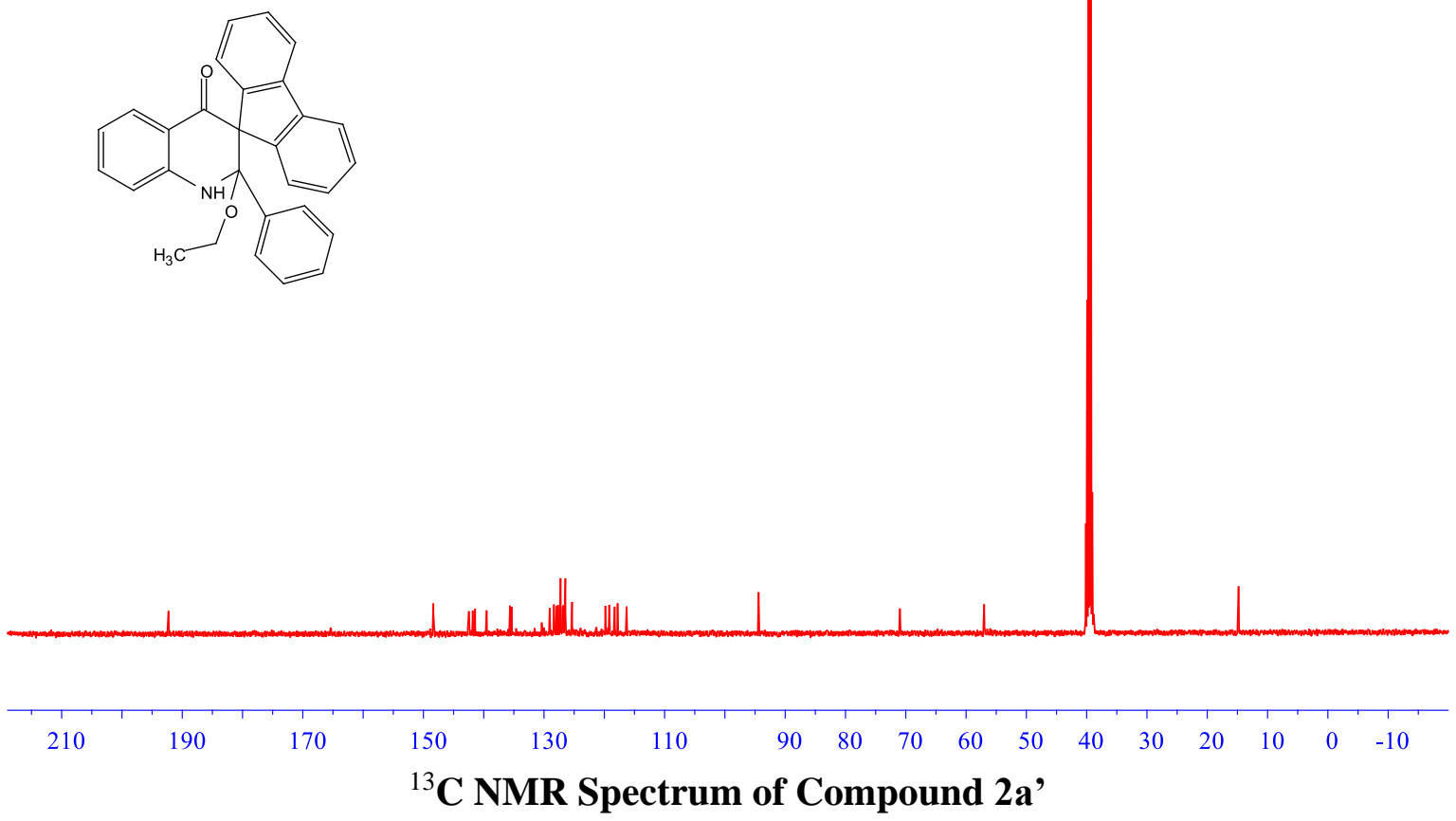


\section{General procedure for synthesis of 1a-1t}

Method A:

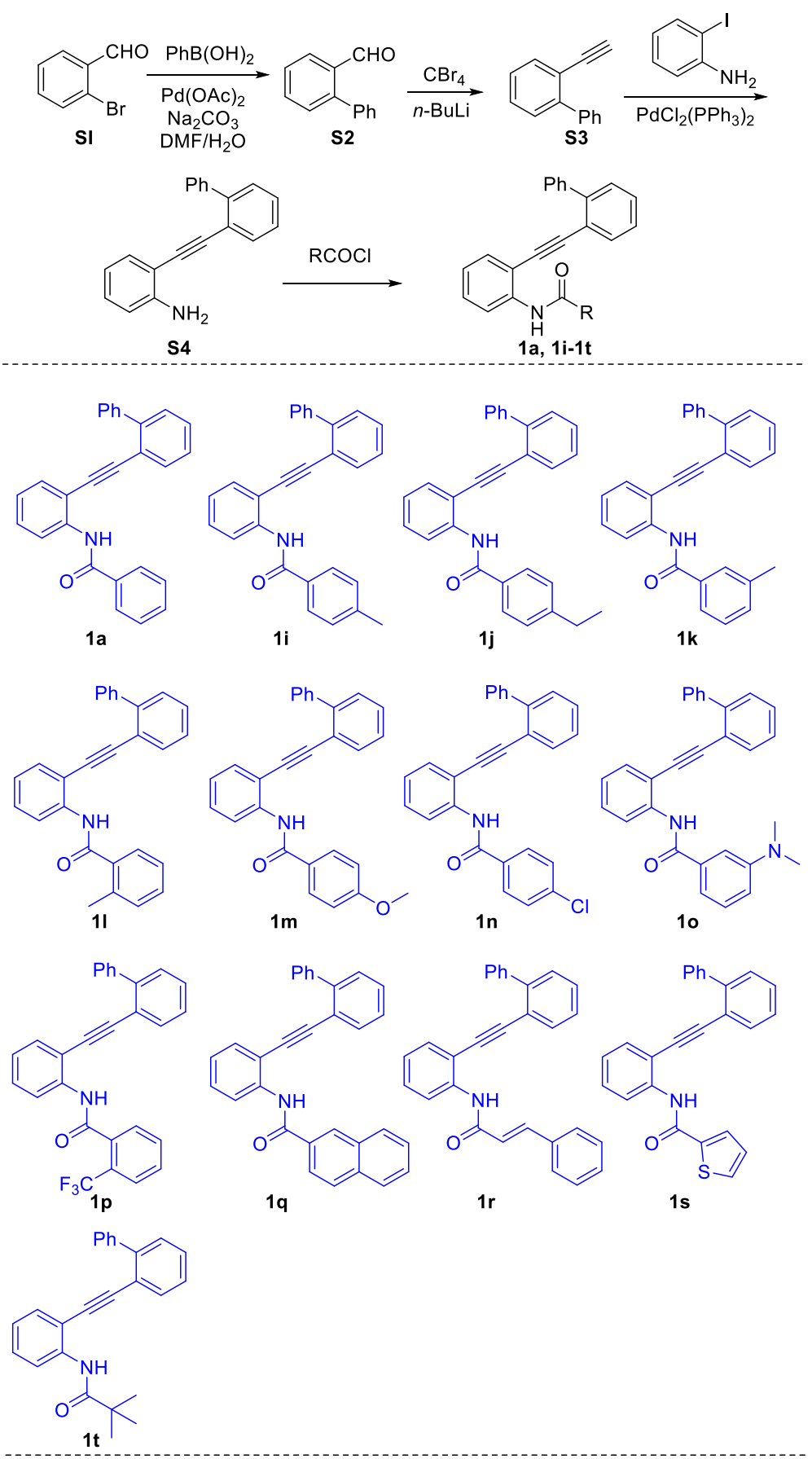

Representative Procedure for Method A. Synthesis of 1a: To $80 \mathrm{~mL}$ of a 2:1 DMF/ $\mathrm{H}_{2} \mathrm{O}$ solution containing 2-bromobenzaldehyde (S1, $7.36 \mathrm{~g}, 40 \mathrm{mmol}, 1.0$ equiv), and $\mathrm{Na}_{2} \mathrm{CO}_{3}(4.24 \mathrm{~g}$, $40 \mathrm{mmol}, 1.0$ equiv) were added phenylboronic acid (4.88 g, $40 \mathrm{mmol}, 1.0$ equiv) and the reaction mixture was stirred for $2 \mathrm{~min} . \mathrm{Pd}(\mathrm{OAc})_{2}(448 \mathrm{mg}, 2 \mathrm{mmol}, 0.05$ equiv) was then added and the flask was flushed with Ar, sealed and allowed to stir at $25{ }^{\circ} \mathrm{C}$ for $12 \mathrm{~h}$. The reaction mixture was extracted with ethyl ether $(2 \times 10 \mathrm{~mL})$. The combined ether layers were dried over anhydrous $\mathrm{Na}_{2} \mathrm{SO}_{4}$ and concentrated under vacuum to yield the crude product, which was purified 
by flash chromatography on silica gel using ethyl acetate/ $n$-penta $=1: 30$ as eluent affording S2 $6.4 \mathrm{~g}(88 \%)$.

To a solution of aldehyde (1.0 equiv) and $\mathrm{CBr}_{4}$ (1.5 equiv) in anhydrous DCM cooled to $0{ }^{\circ} \mathrm{C}$ (ice-water bath) was added $\mathrm{Ph}_{3} \mathrm{P}$ (3.0 equiv) as a solid in small portions. The resultant light-yellow reaction mixture was then stirred at ambient temperature for 3-5 h. Solvent was removed in vacuo, and the residue was dissolved in petroleum ether/EtOAc (60:1). Triphenylphosphine oxide was filtered off by suction. The filtrate was concentrated under reduced pressure, and the crude gem-dibromide was purified by chromatography on silica gel using petroleum ether/EtOAc as the eluent. A well-stirred solution of the gem-dibromide (10 mmol) in anhydrous THF was cooled to $-40{ }^{\circ} \mathrm{C}$ under an argonatmosphere. $n$-BuLi (1.6 M solution in hexanes, 2.05 equiv) was then added dropwise via syringe. The stirring was continued at $-40{ }^{\circ} \mathrm{C}$ until the reaction was complete as monitored by TLC $(2-3 \mathrm{~h})$. After completion of the reaction, the resultant light-yellow/orange mixture was diluted with distilled water, and the stirring was continued for $0.5 \mathrm{~h}$ to allow the mixture to slowly reach room temperature. The layers were separated, and the aqueous layer was extracted with $\mathrm{Et}_{2} \mathrm{O}$. The combined organic layers were washed with brine, dried over anhydrous $\mathrm{Na}_{2} \mathrm{SO}_{4}$, filtered, and concentrated under reduced pressure. The crude product was purified by chromatography on silica gel using petroleum ether/EtOAc as the eluent affording $\mathbf{S 3}$.

2-iodoaniline (5 mmol), $\mathrm{PdCl}_{2}\left(\mathrm{PPh}_{3}\right)_{2}(175 \mathrm{mg}, 0.25 \mathrm{mmol}, 5 \mathrm{~mol} \%)$ and $\mathrm{CuI}(48 \mathrm{mg}, 0.25$ $\mathrm{mmol}, 5 \mathrm{~mol} \%$ ) were placed in a round bottomed flask, then the flask was sealed with rubber septa, evacuated and charged with argon. Diisopropylamine $(20 \mathrm{ml})$ was added to the flask followed by $\mathbf{S 3}(7.5 \mathrm{mmol})$. The reaction mixture was stirred under argon at $50{ }^{\circ} \mathrm{C}$ until the diappearance of the starting material (TLC check, 0.5-2 h). The reaction mixture was cooled to room temperature, diluted with diethyl ether $(20 \mathrm{~mL})$, neutralised with $1 \mathrm{M} \mathrm{HCl}$, the aqueous phase was extracted with diethyl ether $(20 \mathrm{~mL})$. The combined organic phases were washed with water $(40 \mathrm{~mL})$, dried over $\mathrm{Na}_{2} \mathrm{SO}_{4}$ and evaporated in vacuo. The crude residue was purified by coloumn chromatography affording $\mathbf{S 4}$.

S4 $(2 \mathrm{mmol})$ and triethyamine $(2.2 \mathrm{mmol}, 1.1$ eq. $)$ were solubilised in dichloromethane $(5 \mathrm{~mL})$, and cooled to $0{ }^{\circ} \mathrm{C}$. A solution of benzoyl chloride (2.2 mmol, 1.1 eq.) in dichloromethane ( $\left.2 \mathrm{ml}\right)$ was added dropwise. The reaction was stirred at room temperature for 8-24 hours (TLC check). The reaction mixture was poured into water $(20 \mathrm{~mL})$, extracted with dichloromethane $(3 * 15 \mathrm{~mL})$. The combined organic phases were washed with brine $(2 * 20 \mathrm{ml})$, dried over $\mathrm{MgSO}_{4}$ and evaporated under reduced pressure. The crude residue was purified by column chromatography on silica gel affording $\mathbf{1 a}$. 


\section{Method B:}

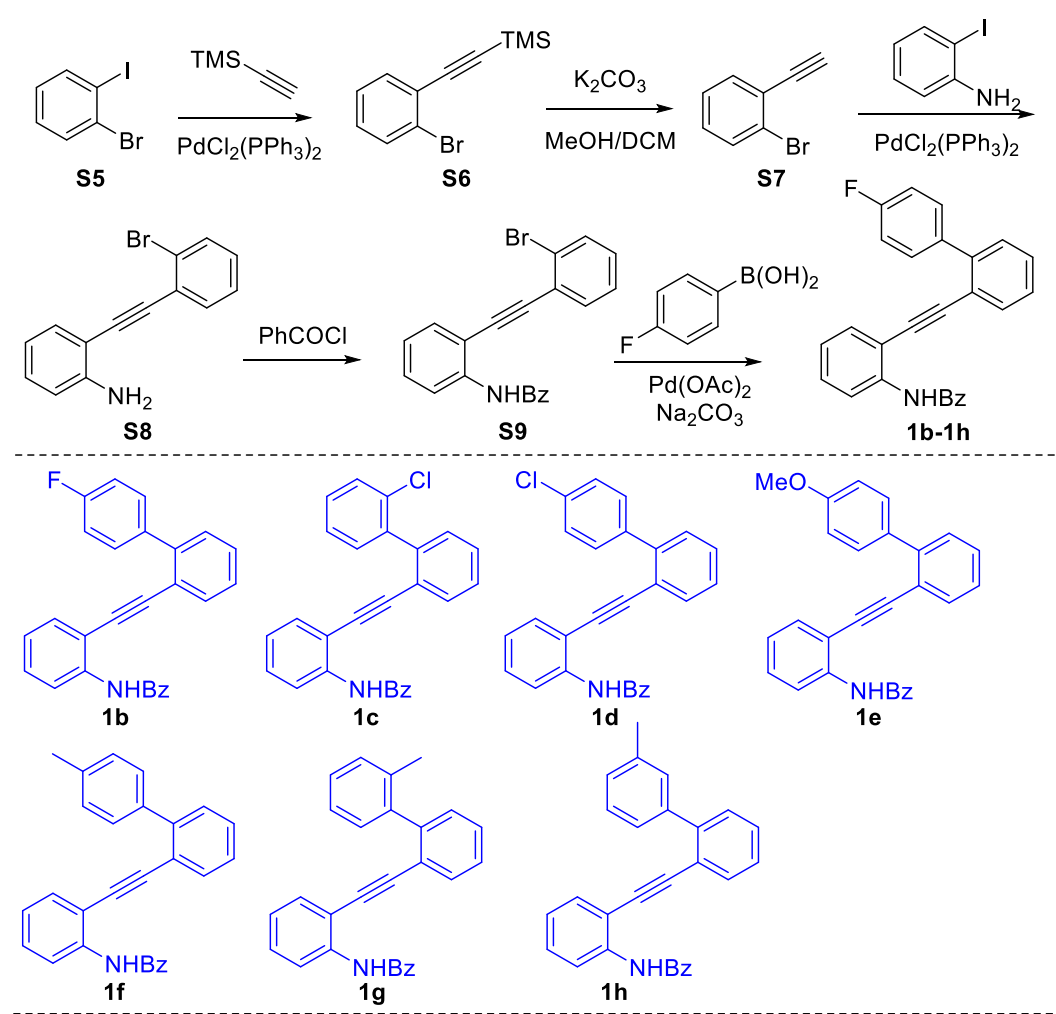

Representative Procedure for Method B. Synthesis of 1B: To a diethylamine solution (60 $\mathrm{mL}$ ) of 2-bromoiodobenzene (S5, $6.0 \mathrm{~g}, 21 \mathrm{mmol})$ was added $\mathrm{PdCl}_{2}\left(\mathrm{PPh}_{3}\right)_{2}(297 \mathrm{mg}, 0.42 \mathrm{mmol})$ and $\mathrm{CuI}(81 \mathrm{mg}, 0.42 \mathrm{mmol})$ at room temperature, and the mixture was stirred for $5 \mathrm{~min}$ before addition of trimethylsilylacetylene. The solution was stirred for $12 \mathrm{~h}$, and the solvent was concentrated in vacuo, added with a saturated $\mathrm{NaHCO}_{3}$ solution, and extracted with hexane. The hexane extract was washed with a saturated $\mathrm{NaCl}$ solution, dried over anhydrous $\mathrm{MgSO}_{4}$, and concentrated under reduced pressure. The residues were chromatographed through a silica gel column (hexane, $\mathrm{Rf}=0.8$ ) to afford compound $\mathbf{S 6}$ as a yellow oil.

To a $\mathrm{CH}_{3} \mathrm{OH} / \mathrm{CH}_{2} \mathrm{Cl}_{2}(15.0 \mathrm{~mL}, 2: 1 \mathrm{v} / \mathrm{v})$ mixing solvent of compound $\mathbf{S 6}$ was added $\mathrm{K}_{2} \mathrm{CO}_{3}$ (4.5 gm, $18 \mathrm{mmol}$ ), and the mixtures were stirred at room temperature for $30 \mathrm{~min}$ before treatment with water. The organic layer was extracted with hexane, washed with a saturated $\mathrm{NaCl}$ solution, and dried over anhydrous $\mathrm{MgSO}_{4}$. The residues were eluted through a silica column (hexane, $\mathrm{Rf}=0.8$ ) to afford the desired product $\mathbf{S 7}$ as an oil.

2-iodoaniline (5 mmol), $\mathrm{PdCl}_{2}\left(\mathrm{PPh}_{3}\right)_{2}(175 \mathrm{mg}, 0.25 \mathrm{mmol}, 5 \mathrm{~mol} \%)$ and $\mathrm{CuI}(48 \mathrm{mg}, 0.25$ mmol, $5 \mathrm{~mol} \%$ ) were placed in a round bottomed flask, then the flask was sealed with rubber septa, evacuated and charged with argon. Diisopropylamine $(20 \mathrm{ml})$ was added to the flask followed by $\mathbf{S} 7(7.5 \mathrm{mmol})$. The reaction mixture was stirred under argon at $50{ }^{\circ} \mathrm{C}$ until the diappearance of the starting material (TLC check, 0.5-2 h). The reaction mixture was cooled to room temperature, diluted with diethyl ether $(20 \mathrm{~mL})$, neutralised with $1 \mathrm{M} \mathrm{HCl}$, the aqueous phase was extracted with diethyl ether $(20 \mathrm{~mL})$. The combined organic phases were washed with water $(40 \mathrm{~mL})$, dried over $\mathrm{Na}_{2} \mathrm{SO}_{4}$ and evaporated in vacuo. The crude residue was purified by coloumn chromatography affording $\mathbf{S 8}$.

S8 $(3 \mathrm{mmol})$ and triethyamine $(3.3 \mathrm{mmol}, 1.1$ eq.) were solubilised in dichloromethane (10 
$\mathrm{mL}$ ), and cooled to $0{ }^{\circ} \mathrm{C}$. A solution of benzoyl chloride (3.3 mmol, 1.1 eq.) in dichloromethane $(2 \mathrm{ml})$ was added dropwise. The reaction was stirred at room temperature for 8-24 hours (TLC check). The reaction mixture was poured into water $(20 \mathrm{~mL})$, extracted with dichloromethane $(3 * 15 \mathrm{~mL})$. The combined organic phases were washed with brine $(2 * 20 \mathrm{ml})$, dried over $\mathrm{MgSO}_{4}$ and evaporated under reduced pressure. The crude residue was purified by column chromatography on silica gel affording $\mathbf{S 9}$.

To an oven-dried three necked flask, S9 (2 mmol), (4-fluorophenyl)boronic acid (2.4 mmol), aqueous solution of $\mathrm{K}_{2} \mathrm{CO}_{3}(2 \mathrm{M}, 5 \mathrm{~mL})$ and DME $(4 \mathrm{~mL})$ were added under a gentle stream of nitrogen, and the mixture was stirred for $30 \mathrm{~min}$ at room temperature under $\mathrm{N}_{2}$ atmosphere. To the stirred mixture, $\mathrm{PdCl}_{2}\left(\mathrm{PPh}_{3}\right)_{2}(0.040 \mathrm{mmol})$ was added at room temperature, and the mixture was stirred for overnight at $80{ }^{\circ} \mathrm{C}$, under $\mathrm{N}_{2}$. The reaction mixture was then cooled to room temperature and diluted with EtOAc. The organic layer was washed with water and dried over $\mathrm{MgSO}_{4}$. After removing the volatiles in vacuo, the residue was subjected to column chromatography on silica gel (hexane/EtOAc $=30 / 1$ ) to afford $\mathbf{1 b}$. 


\section{Characterization Data of Compounds 1a-1t:}

N-(2-([1,1'-biphenyl]-2-ylethynyl)phenyl)benzamide (1a)

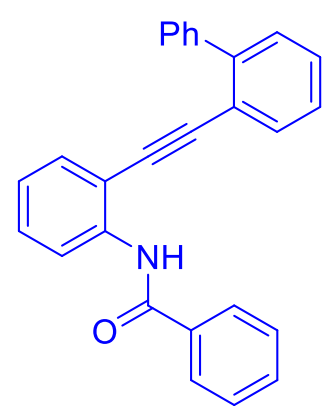

White Solid,

${ }^{1} \mathbf{H}$ NMR (400 MHz, CDCl $) \delta 8.52-8.46(\mathrm{~m}, 2 \mathrm{H}), 7.63-7.55(\mathrm{~m}, 3 \mathrm{H})$, $7.54-7.50(\mathrm{~m}, 2 \mathrm{H}), 7.45(\mathrm{t}, J=7.4 \mathrm{~Hz}, 1 \mathrm{H}), 7.40-7.35(\mathrm{~m}, 3 \mathrm{H}), 7.35-$ $7.28(\mathrm{~m}, 4 \mathrm{H}), 7.19$ (t, $J=7.4 \mathrm{~Hz}, 2 \mathrm{H}), 7.12(\mathrm{t}, J=7.3 \mathrm{~Hz}, 1 \mathrm{H}), 6.99$ (td, $J=$ 7.6, $0.7 \mathrm{~Hz}, 1 \mathrm{H})$;

${ }^{13}$ C NMR (100 MHz, $\left.\mathbf{C D C l}_{3}\right) \delta 164.86,143.29,139.49,138.38,134.31$, $132.42,131.39,131.30,129.31,129.29,128.82,128.55,128.39,127.61$, $127.31,126.78,126.55,123.04,120.16,118.69,111.96,96.24,86.78,76.90$,

$76.58,76.26$.

N-(2-((4'-fluoro-[1,1'-biphenyl]-2-yl)ethynyl)phenyl)benzamide (1b)

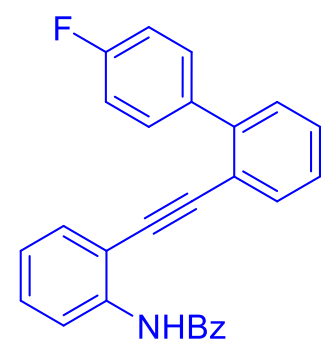

White Solid;

${ }^{1}{ }^{H}$ NMR ${ }^{1} \mathbf{H}$ NMR (400 MHz, $\left.\mathbf{C D C l}_{3}\right) \delta 8.62-8.52(\mathrm{~m}, 2 \mathrm{H}), 7.76-7.71$ $(\mathrm{m}, 2 \mathrm{H}), 7.64(\mathrm{dd}, J=7.6,0.9 \mathrm{~Hz}, 1 \mathrm{H}), 7.58-7.51(\mathrm{~m}, 3 \mathrm{H}), 7.48-7.35(\mathrm{~m}$, $7 \mathrm{H}), 7.08(\mathrm{td}, J=7.6,1.1 \mathrm{~Hz}, 1 \mathrm{H}), 6.97-6.90(\mathrm{~m}, 2 \mathrm{H})$;

${ }^{13}$ C NMR (100 MHz, $\left.\mathbf{C D C l}_{3}\right) \delta 164.80,163.15,160.69,142.22,138.41$, $135.51,135.48,134.35,132.43,131.45,131.21,130.29,130.21,129.41$, $129.24,128.85,128.39,126.89,126.50,123.08,120.15,118.73,114.64$, $114.43,111.78,96.01,87.00,76.88,76.56,76.25$;

N-(2-((2'-chloro-[1,1'-biphenyl]-2-yl)ethynyl)phenyl)benzamide (1c)

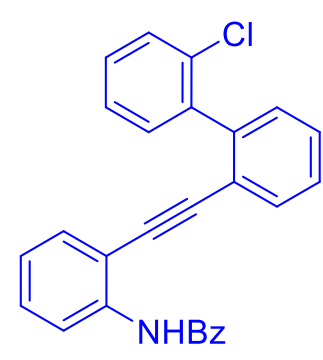

White Solid;

${ }^{1} \mathbf{H}$ NMR (400 MHz, CDCl$) \delta 8.53(\mathrm{~d}, J=8.6 \mathrm{~Hz}, 2 \mathrm{H}), 7.83(\mathrm{dd}, J=5.2$, $3.3 \mathrm{~Hz}, 2 \mathrm{H}), 7.67-7.61(\mathrm{~m}, 1 \mathrm{H}), 7.60-7.54(\mathrm{~m}, 1 \mathrm{H}), 7.52-7.46(\mathrm{~m}, 2 \mathrm{H})$, $7.45-7.28(\mathrm{~m}, 6 \mathrm{H}), 7.18(\mathrm{ddd}, J=7.4,4.7,1.9 \mathrm{~Hz}, 2 \mathrm{H}), 7.11(\mathrm{td}, J=7.4$, $1.4 \mathrm{~Hz}, 1 \mathrm{H}), 7.00(\mathrm{td}, J=7.6,1.1 \mathrm{~Hz}, 1 \mathrm{H})$;

${ }^{13}$ C NMR (100 MHz, CDCl $) \delta 164.87,141.40,138.57,138.33,134.51$, $132.62,131.56,131.45,130.91,129.70,129.30,129.02,128.66,128.44$, $128.25,127.45,126.68,125.86,122.99,121.66,118.68,111.82,95.23,87.00,76.93,76.61$, 76.29 ;

N-(2-((4'-chloro-[1,1'-biphenyl]-2-yl)ethynyl)phenyl)benzamide (1d)

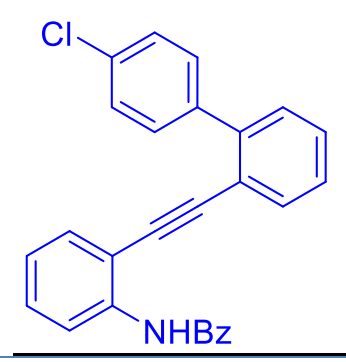

White Solid;

${ }^{1}$ H NMR (400 MHz, CDCl $) \delta 8.61-8.52(\mathrm{~m}, 2 \mathrm{H}), 7.76-7.69(\mathrm{~m}, 2 \mathrm{H})$, $7.64(\mathrm{dd}, J=7.5,0.9 \mathrm{~Hz}, 1 \mathrm{H}), 7.57-7.48(\mathrm{~m}, 3 \mathrm{H}), 7.45-7.34(\mathrm{~m}, 7 \mathrm{H}), 7.24$ $-7.19(\mathrm{~m}, 2 \mathrm{H}), 7.08(\mathrm{td}, J=7.6,1.0 \mathrm{~Hz}, 1 \mathrm{H})$; 
${ }^{13}$ C NMR (101 MHz, CDCl $) \delta 164.79,141.96,138.46,137.90,134.33,133.42,132.52,131.46$, $131.22,129.88,129.47,129.18,128.90,128.40,127.80,127.12,126.51,123.09,120.09,118.74$, $111.71,95.85,87.16,76.90,76.58,76.26$;

\section{N-(2-((4'-methoxy-[1,1'-biphenyl]-2-yl)ethynyl)phenyl)benzamide (1e)}

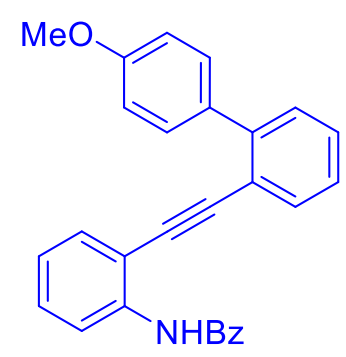

White Solid;

${ }^{1}$ H NMR (400 MHz, CDCl $) \delta 8.66-8.53(\mathrm{~m}, 2 \mathrm{H}), 7.71-7.66(\mathrm{~m}, 2 \mathrm{H})$, $7.63(\mathrm{~d}, J=7.6 \mathrm{~Hz}, 1 \mathrm{H}), 7.58-7.49(\mathrm{~m}, 3 \mathrm{H}), 7.45-7.35(\mathrm{~m}, 6 \mathrm{H}), 7.32(\mathrm{dt}$, $J=7.7,4.4 \mathrm{~Hz}, 1 \mathrm{H}), 7.07(\mathrm{td}, J=7.6,1.1 \mathrm{~Hz}, 1 \mathrm{H}), 6.81-6.75(\mathrm{~m}, 2 \mathrm{H})$, $3.71(\mathrm{~s}, 3 \mathrm{H})$;

${ }^{13}$ C NMR (100 MHz, CDCl $) \delta 164.75,158.79,142.84,138.41,134.23$, $132.55,131.87,131.34,131.29,129.70,129.24,129.16,128.81,128.36$, $126.48,126.32$, 123.01, 119.92, 118.67, 113.06, 112.02, 96.53, 86.72, 76.90, 76.58, 76.26, 54.72;

\section{N-(2-((4'-methyl-[1,1'-biphenyl]-2-yl)ethynyl)phenyl)benzamide (1f)}

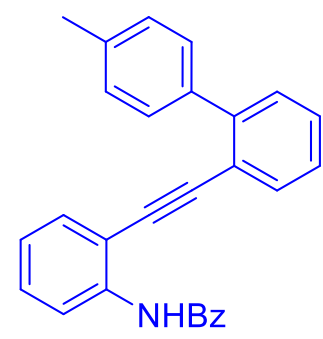

\section{White Solid;}

${ }^{1}$ H NMR (400 MHz, CDCl $) \delta 8.51(\mathrm{~d}, J=8.0 \mathrm{~Hz}, 2 \mathrm{H}), 7.56(\mathrm{~d}, J=8.0 \mathrm{~Hz}$, $3 \mathrm{H}), 7.43(\mathrm{~d}, J=8.0 \mathrm{~Hz}, 3 \mathrm{H}), 7.38-7.33(\mathrm{~m}, 4 \mathrm{H}), 7.29(\mathrm{dd}, J=13.9,5.7 \mathrm{~Hz}$, $3 \mathrm{H}), 6.99(\mathrm{dd}, J=14.3,7.7 \mathrm{~Hz}, 3 \mathrm{H}), 2.14(\mathrm{~s}, 3 \mathrm{H})$;

${ }^{13}$ C NMR (100 MHz, $\left.\mathbf{C D C l}_{3}\right) \delta 164.82,143.16,138.41,137.16,136.56$, $134.21,132.54,131.35,131.31,129.26,128.81,128.41,128.36,126.53$, $126.48,123.01,120.02,118.64,112.00,96.42,86.72,76.90,76.58,76.27$, 20.69

\section{N-(2-((2'-methyl-[1,1'-biphenyl]-2-yl)ethynyl)phenyl)benzamide (1g)}

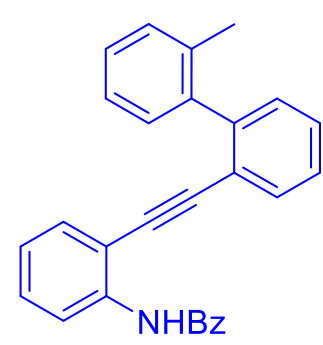

\section{White Solid;}

${ }^{1} \mathbf{H}$ NMR (400 MHz, CDCl $) \delta 8.41(\mathrm{~d}, J=8.1 \mathrm{~Hz}, 2 \mathrm{H}), 7.72(\mathrm{~d}, J=8.6 \mathrm{~Hz}$, $2 \mathrm{H}), 7.49(\mathrm{dd}, J=7.6,1.2 \mathrm{~Hz}, 1 \mathrm{H}), 7.47-7.41(\mathrm{~m}, 1 \mathrm{H}), 7.40-7.34(\mathrm{~m}, 2 \mathrm{H})$, $7.30(\mathrm{td}, J=7.5,1.5 \mathrm{~Hz}, 1 \mathrm{H}), 7.24(\mathrm{dt}, J=7.5,3.8 \mathrm{~Hz}, 1 \mathrm{H}), 7.19$ (ddd, $J=$ $7.4,6.2,1.6 \mathrm{~Hz}, 2 \mathrm{H}), 7.07-7.03(\mathrm{~m}, 3 \mathrm{H}), 7.00(\mathrm{dd}, J=7.7,1.5 \mathrm{~Hz}, 1 \mathrm{H})$, $6.93-6.86(\mathrm{~m}, 2 \mathrm{H}), 2.05(\mathrm{~s}, 3 \mathrm{H})$;

${ }^{13}$ C NMR (100 MHz, CDCl $) \delta 164.99,144.19,139.74,138.24,135.51$, $134.56,131.61,131.45,131.27,129.45,129.43,129.23,129.13,128.45,128.43,127.35,126.78$, $126.76,124.90,123.09,121.55,118.71,112.02$, 95.84, 86.69, 77.01, 76.70, 76.38, 19.61; 


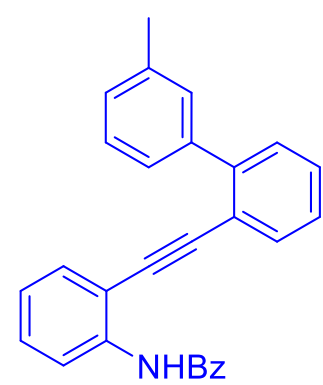

White Solid;

${ }^{1} \mathbf{H}$ NMR (400 MHz, CDCl $) \delta 8.69-8.55(\mathrm{~m}, 2 \mathrm{H}), 7.73-7.67(\mathrm{~m}, 2 \mathrm{H})$, $7.66-7.62(\mathrm{~m}, 1 \mathrm{H}), 7.52$ (ddd, $J=6.9,4.0,1.2 \mathrm{~Hz}, 1 \mathrm{H}), 7.46-7.43(\mathrm{~m}, 3 \mathrm{H})$, $7.43-7.31(\mathrm{~m}, 6 \mathrm{H}), 7.15(\mathrm{t}, J=7.6 \mathrm{~Hz}, 1 \mathrm{H}), 7.07(\mathrm{td}, J=7.6,1.1 \mathrm{~Hz}, 1 \mathrm{H})$, $7.00(\mathrm{~d}, J=7.5 \mathrm{~Hz}, 1 \mathrm{H}), 2.29(\mathrm{~s}, 3 \mathrm{H})$;

${ }^{13}$ C NMR (101 MHz, CDCl $) \delta 164.79,143.33,139.39,138.36,137.19$, $134.21,132.41,131.37,131.25,129.30,129.26,128.79,128.35,128.05$, $127.51,126.67,126.53,125.66,123.04,120.08,118.68,112.02,96.43$,

$86.85,76.89,76.57,76.26,21.02$;

\section{N-(2-([1,1'-biphenyl]-2-ylethynyl)phenyl)-4-methylbenzamide (1i)}

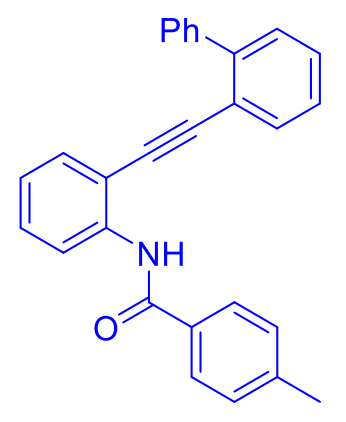

White Solid;

${ }^{1} \mathbf{H}$ NMR (400 MHz, $\left.\mathbf{C D C l}_{3}\right) \delta 8.57(\mathrm{~d}, J=8.3 \mathrm{~Hz}, 2 \mathrm{H}), 7.66-7.53(\mathrm{~m}, 5 \mathrm{H})$, $7.45-7.37(\mathrm{~m}, 2 \mathrm{H}), 7.36-7.29(\mathrm{~m}, 3 \mathrm{H}), 7.26(\mathrm{t}, J=7.5 \mathrm{~Hz}, 2 \mathrm{H}), 7.18(\mathrm{t}, J$ $=7.9 \mathrm{~Hz}, 3 \mathrm{H}), 7.01(\mathrm{td}, J=7.6,0.9 \mathrm{~Hz}, 1 \mathrm{H}), 2.38(\mathrm{~s}, 3 \mathrm{H})$;

${ }^{13}$ C NMR (100 MHz, CDCl $) \delta 164.77,143.32,141.92,139.58,138.60$, $132.46,131.56,131.32,129.35,129.29,129.08,128.83,128.63,127.64$, $127.34,126.83,126.61,122.91,120.24,118.74,111.94,96.26,86.94,77.05$, $76.73,76.41,21.11$;

\section{N-(2-([1,1'-biphenyl]-2-ylethynyl)phenyl)-4-ethylbenzamide (1j)}

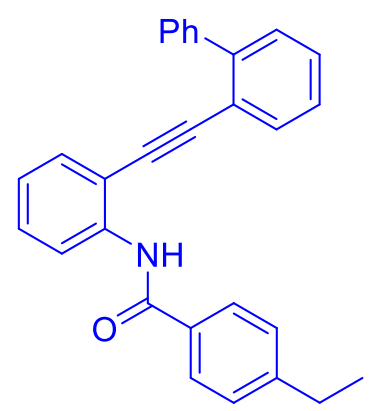

\section{White Solid;}

${ }^{1} \mathbf{H}$ NMR (400 MHz, CDCl $) \delta 8.56(\mathrm{~d}, J=7.9 \mathrm{~Hz}, 2 \mathrm{H}), 7.62(\mathrm{ddd}, J=$ 9.9, 7.0, 4.6 Hz, 5H), $7.48-7.42(\mathrm{~m}, 2 \mathrm{H}), 7.39-7.32(\mathrm{~m}, 3 \mathrm{H}), 7.32-$ $7.19(\mathrm{~m}, 5 \mathrm{H}), 7.08-7.01(\mathrm{~m}, 1 \mathrm{H}), 2.72(\mathrm{q}, J=7.6 \mathrm{~Hz}, 2 \mathrm{H}), 1.28(\mathrm{t}, J=$ $7.6 \mathrm{~Hz}, 3 \mathrm{H})$;

${ }^{13}$ C NMR (101 MHz, CDCl $) \delta 164.81,148.11,143.31,139.53,138.54$, $132.43,131.73,131.28,129.30,129.26,128.76,128.57,127.86,127.59$, $127.29,126.77,126.67,122.86,120.24,118.67,111.86,96.15,86.88$, $76.89,76.58,76.26,28.37,14.88$;

\section{N-(2-([1,1'-biphenyl]-2-ylethynyl)phenyl)-3-methylbenzamide (1k)}

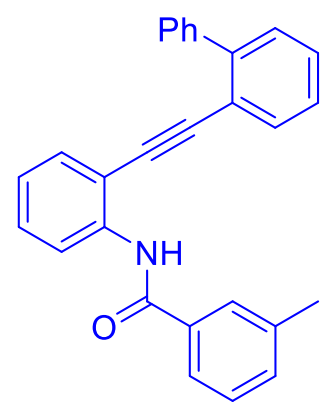

White Solid;

${ }^{1} \mathbf{H}$ NMR (400 MHz, CDCl $) \delta 8.68-8.52(\mathrm{~m}, 2 \mathrm{H}), 7.65(\mathrm{~d}, J=6.6 \mathrm{~Hz}, 2 \mathrm{H})$, $7.58(\mathrm{~d}, J=7.2 \mathrm{~Hz}, 2 \mathrm{H}), 7.45(\mathrm{dd}, J=14.5,5.1 \mathrm{~Hz}, 3 \mathrm{H}), 7.39-7.21(\mathrm{~m}, 8 \mathrm{H})$, $7.04(\mathrm{t}, J=7.4 \mathrm{~Hz}, 1 \mathrm{H}), 2.37(\mathrm{~s}, 3 \mathrm{H})$;

${ }^{13}$ C NMR (101 MHz, CDCl 3$) \delta 164.99,143.41,139.50,138.52,138.18$, $134.39,132.32,132.22,131.17,129.31,128.80,128.55,128.32,127.56$, $127.32,127.28,126.75,123.58,122.95,120.21,118.64,111.91,96.29$, 
N-(2-([1,1'-biphenyl]-2-ylethynyl)phenyl)-2-methylbenzamide (11)<smiles>Cc1ccccc1C(=O)Nc1ccccc1C#Cc1ccccc1-c1ccccc1</smiles>

White Solid;

${ }^{1} \mathrm{H}$ NMR (400 MHz, CDCl $\left.{ }_{3}\right) \delta 8.55(\mathrm{~d}, J=8.5 \mathrm{~Hz}, 1 \mathrm{H}), 8.10(\mathrm{~s}, 1 \mathrm{H}), 7.53$ $(\mathrm{d}, J=7.5 \mathrm{~Hz}, 1 \mathrm{H}), 7.49-7.44(\mathrm{~m}, 2 \mathrm{H}), 7.41-7.30(\mathrm{~m}, 7 \mathrm{H}), 7.26(\mathrm{~d}, J=$ $7.5 \mathrm{~Hz}, 1 \mathrm{H}), 7.20(\mathrm{dd}, J=8.1,4.7 \mathrm{~Hz}, 4 \mathrm{H}), 7.09-7.02(\mathrm{~m}, 1 \mathrm{H}), 2.50$ (s, $3 \mathrm{H})$;

${ }^{13}$ C NMR (100 MHz, $\left.\mathbf{C D C l}_{3}\right) \delta 167.28,143.40,139.53,138.48,136.50$, $135.55,132.30,131.35,131.04,130.03,129.19,128.66,128.46,127.43$, $127.12,126.71,126.39,125.57,123.09,120.22,118.80,111.98,96.00$, $86.69,76.90,76.58,76.26,19.67$;

N-(2-([1,1'-biphenyl]-2-ylethynyl)phenyl)-4-methoxybenzamide (1m)<smiles>COc1ccc(C(=O)Nc2ccccc2C#Cc2ccccc2-c2ccccc2)cc1</smiles>

White Solid;

${ }^{1}$ H NMR (400 MHz, CDCl $) \delta 8.54(\mathrm{~d}, J=8.2 \mathrm{~Hz}, 1 \mathrm{H}), 8.49(\mathrm{~s}, 1 \mathrm{H})$, $7.68-7.59(\mathrm{~m}, 5 \mathrm{H}), 7.48-7.41(\mathrm{~m}, 2 \mathrm{H}), 7.35(\mathrm{ddd}, J=9.5,4.6,2.2 \mathrm{~Hz}$, $3 \mathrm{H}), 7.30$ (dd, $J=10.3,4.7 \mathrm{~Hz}, 2 \mathrm{H}), 7.24-7.19(\mathrm{~m}, 1 \mathrm{H}), 7.04$ (td, $J=7.6$, $1.0 \mathrm{~Hz}, 1 \mathrm{H}), 6.92-6.85(\mathrm{~m}, 2 \mathrm{H}), 3.86(\mathrm{~s}, 3 \mathrm{H})$;

${ }^{13}$ C NMR (100 MHz, CDCl$\left._{3}\right) \delta 164.35,161.99,143.23,139.53,138.61$, $132.46,131.27,129.32,129.27,128.78,128.57,128.42,127.62,127.32$, $126.79,126.62,122.75,120.22,118.63,113.58,111.79,96.11,86.95$, $76.90,76.58,76.27,55.01$;

\section{N-(2-([1,1'-biphenyl]-2-ylethynyl)phenyl)-4-chlorobenzamide (1n)}<smiles>O=C(Nc1ccccc1C#Cc1ccccc1-c1ccccc1)c1ccc(Cl)cc1</smiles>

White Solid;

${ }^{1} \mathbf{H}$ NMR (400 MHz, $\left.\mathbf{C D C l}_{3}\right) \delta 8.55-8.41(\mathrm{~m}, 2 \mathrm{H}), 7.67-7.59(\mathrm{~m}, 3 \mathrm{H})$, $7.56-7.50(\mathrm{~m}, 2 \mathrm{H}), 7.46(\mathrm{dd}, J=4.8,0.9 \mathrm{~Hz}, 2 \mathrm{H}), 7.43-7.40(\mathrm{~m}, 1 \mathrm{H})$, $7.40-7.33(\mathrm{~m}, 4 \mathrm{H}), 7.27(\mathrm{dd}, J=10.6,4.7 \mathrm{~Hz}, 2 \mathrm{H}), 7.15(\mathrm{t}, J=7.4 \mathrm{~Hz}$, $1 \mathrm{H}), 7.08(\mathrm{td}, J=7.6,1.1 \mathrm{~Hz}, 1 \mathrm{H})$;

${ }^{13}$ C NMR (100 MHz, CDCl $) \delta 163.73,143.06,139.43,138.11,137.57$, $132.67,132.54,131.35,129.37,129.30,128.93,128.61,128.50,127.92$, $127.63,127.32,126.85,123.21,119.99,118.69,111.99,96.34,86.75$,

$76.89,76.57,76.25$; 


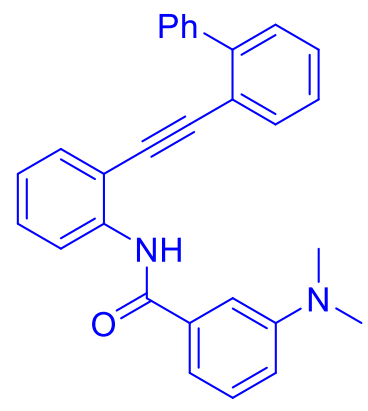

White Solid;

${ }^{1} \mathbf{H}$ NMR $(400 \text { MHz, CDCl})_{3} \delta 8.68-8.55(\mathrm{~m}, 2 \mathrm{H}), 7.64(\mathrm{~d}, J=7.6 \mathrm{~Hz}$, $1 \mathrm{H}), 7.61-7.54(\mathrm{~m}, 2 \mathrm{H}), 7.43(\mathrm{~d}, J=4.0 \mathrm{~Hz}, 2 \mathrm{H}), 7.36-7.26(\mathrm{~m}, 5 \mathrm{H})$, $7.25-7.19(\mathrm{~m}, 3 \mathrm{H}), 7.04(\mathrm{t}, J=7.3 \mathrm{~Hz}, 1 \mathrm{H}), 6.95-6.83(\mathrm{~m}, 2 \mathrm{H}), 2.94(\mathrm{~s}$, $6 \mathrm{H})$;

${ }^{13}$ C NMR (100 MHz, $\left.\mathbf{C D C l}_{3}\right) \delta 165.73,150.32,143.44,139.50,138.69$, $135.27,132.42,131.18,129.26,128.96,128.72,128.58,127.56,127.27$, $126.71,122.79,120.23,118.54,115.13,113.46,111.84,110.84,96.15$, $86.81,76.90,76.58,76.27,39.92$;

\section{N-(2-([1,1'-biphenyl]-2-ylethynyl)phenyl)-2-(trifluoromethyl)benzamide (1p)}

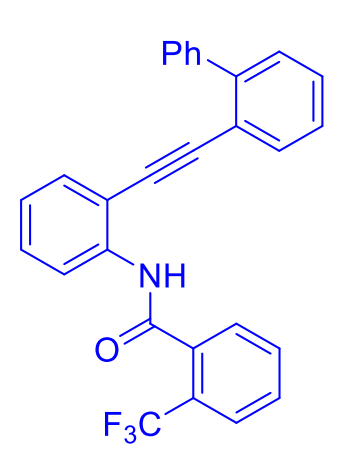

White Solid;

${ }^{1} \mathbf{H}$ NMR $\left(400 \mathrm{MHz}, \mathbf{C D C l}_{3}\right) \delta 8.50(\mathrm{~d}, J=8.3 \mathrm{~Hz}, 1 \mathrm{H}), 7.93(\mathrm{~s}, 1 \mathrm{H}), 7.76$ $(\mathrm{d}, J=7.4 \mathrm{~Hz}, 1 \mathrm{H}), 7.61-7.50(\mathrm{~m}, 3 \mathrm{H}), 7.45-7.34(\mathrm{~m}, 7 \mathrm{H}), 7.32-7.27(\mathrm{~m}$, $1 \mathrm{H}), 7.18-7.08(\mathrm{~m}, 4 \mathrm{H})$;

${ }^{13}$ C NMR (100 MHz, $\left.\mathbf{C D C l}_{3}\right) \delta 165.06,143.23,139.48,137.88,135.11$, $132.33,131.70,131.47,129.81,129.19,129.14,128.68,128.34,127.72$, $127.59,127.34,127.27,127.13,126.72,126.36,126.31,123.66,120.15$, 119.36, 112.36, 96.02, 86.43, 76.89, 76.57, 76.25;

\section{N-(2-([1,1'-biphenyl]-2-ylethynyl)phenyl)-2-naphthamide (1q)}<smiles>O=C(Nc1ccccc1C#Cc1ccccc1-c1ccccc1)c1ccc2ccccc2c1</smiles>

White Solid;

${ }^{1} \mathbf{H}$ NMR (400 MHz, CDCl $) \delta 8.76(\mathrm{~s}, 1 \mathrm{H}), 8.62(\mathrm{~d}, J=8.4 \mathrm{~Hz}, 1 \mathrm{H})$, $8.24(\mathrm{~s}, 1 \mathrm{H}), 7.90-7.86(\mathrm{~m}, 2 \mathrm{H}), 7.83-7.77(\mathrm{~m}, 2 \mathrm{H}), 7.68(\mathrm{~d}, J=7.4 \mathrm{~Hz}$, $1 \mathrm{H}), 7.60-7.51(\mathrm{~m}, 4 \mathrm{H}), 7.45-7.33(\mathrm{~m}, 5 \mathrm{H}), 7.13-7.04(\mathrm{~m}, 4 \mathrm{H})$;

${ }^{13}$ C NMR (100 MHz, CDCl$) ~ \delta 164.75,143.02,138.38,137.02,134.02$, $132.71,132.64,132.21,131.32,131.30,129.70,129.32,128.92,128.22$, $127.67,127.63,127.26,127.12,126.88,126.63,126.27,125.85,125.80$, 123.04, 120.34, 118.69, 111.91, 96.40, 87.14, 76.91, 76.60, 76.28;

\section{N-(2-([1,1'-biphenyl]-2-ylethynyl)phenyl)cinnamamide (1r)}

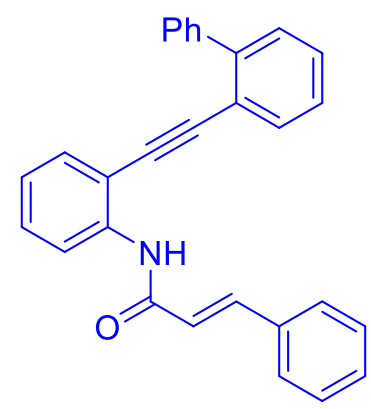

White Solid;

${ }^{1}$ H NMR (400 MHz, $\left.\mathbf{C D C l}_{3}\right) \delta 8.52(\mathrm{~d}, J=8.3 \mathrm{~Hz}, 1 \mathrm{H}), 7.69(\mathrm{ddd}, J=$ 25.0, 13.0, 8.4 Hz, 5H), $7.52-7.33(\mathrm{~m}, 12 \mathrm{H}), 7.25-7.20(\mathrm{~m}, 1 \mathrm{H}), 7.06$ (td, $J=7.6,1.0 \mathrm{~Hz}, 1 \mathrm{H}), 5.74(\mathrm{~d}, J=15.5 \mathrm{~Hz}, 1 \mathrm{H})$;

${ }^{13}$ C NMR (100 MHz, CDCl $) \delta$ 163.28, 142.86, 141.86, 139.89, 138.43, $134.13,132.68,131.30,129.52,129.32,129.22,128.79,128.62,128.26$, $127.90,127.82,127.80,126.93,122.96,120.49,120.28,119.06,111.71$, 
N-(2-([1,1'-biphenyl]-2-ylethynyl)phenyl)thiophene-2-carboxamide (1s)<smiles>O=C(Nc1ccccc1C#Cc1ccccc1-c1ccccc1)c1cccs1</smiles>

White Solid;

${ }^{1} \mathbf{H}$ NMR $\left(400 \mathrm{MHz}, \mathbf{C D C l}_{3}\right) \delta 8.52-8.36(\mathrm{~m}, 2 \mathrm{H}), 7.69(\mathrm{~d}, J=7.8 \mathrm{~Hz}, 1 \mathrm{H})$, $7.67-7.62(\mathrm{~m}, 2 \mathrm{H}), 7.46(\mathrm{ddt}, J=10.6,7.8,3.8 \mathrm{~Hz}, 3 \mathrm{H}), 7.39-7.30(\mathrm{~m}$, $5 \mathrm{H}), 7.23(\mathrm{dd}, J=8.2,6.6 \mathrm{~Hz}, 1 \mathrm{H}), 7.09-7.02(\mathrm{~m}, 2 \mathrm{H}), 6.99(\mathrm{dd}, J=4.9$, $3.8 \mathrm{~Hz}, 1 \mathrm{H})$;

${ }^{13}$ C NMR (100 MHz, CDCl $)$ 159.23, 143.14, 139.56, 138.76, 138.04, $134.40,133.37,132.63,131.36,130.49,129.38,129.29,128.87,128.62$, $127.99,127.72,127.58,127.53,127.42,126.84,123.06,120.10,118.74$, $111.85,96.33,86.71,76.93,76.62,76.30$;

N-(2-([1,1'-biphenyl]-2-ylethynyl)phenyl)pivalamide (1t)<smiles>CC(C)(C)C(=O)Nc1ccccc1C#Cc1ccccc1-c1ccccc1</smiles>

White Solid;

${ }^{1} \mathbf{H}$ NMR $\left(400 \mathrm{MHz}, \mathbf{C D C l}_{3}\right) \delta 8.42(\mathrm{~d}, J=8.8 \mathrm{~Hz}, 1 \mathrm{H}), 8.20(\mathrm{~s}, 1 \mathrm{H}), 7.64$ (dd, $J=11.5,4.4 \mathrm{~Hz}, 3 \mathrm{H}), 7.43(\mathrm{dd}, J=8.2,6.7 \mathrm{~Hz}, 4 \mathrm{H}), 7.39-7.34(\mathrm{~m}$, $2 \mathrm{H}), 7.29(\mathrm{t}, J=7.3 \mathrm{~Hz}, 2 \mathrm{H}), 6.99(\mathrm{dd}, J=11.0,4.2 \mathrm{~Hz}, 1 \mathrm{H}), 1.18(\mathrm{~s}, 9 \mathrm{H})$;

${ }^{13}$ C NMR (101 MHz, $\left.\mathbf{C D C l}_{3}\right) \delta 176.22,143.32,139.75,138.42,132.37$, $131.24,129.43,129.17,128.78,128.75,127.71,127.38,126.78,122.67$, $120.17,118.61,111.77,95.83,86.69,76.92,76.61,76.29,39.50,27.05$; 


\section{Copies of ${ }^{1} \mathrm{H}$ and ${ }^{13} \mathrm{C}$ NMR Spectra for Compounds 1a-1t}
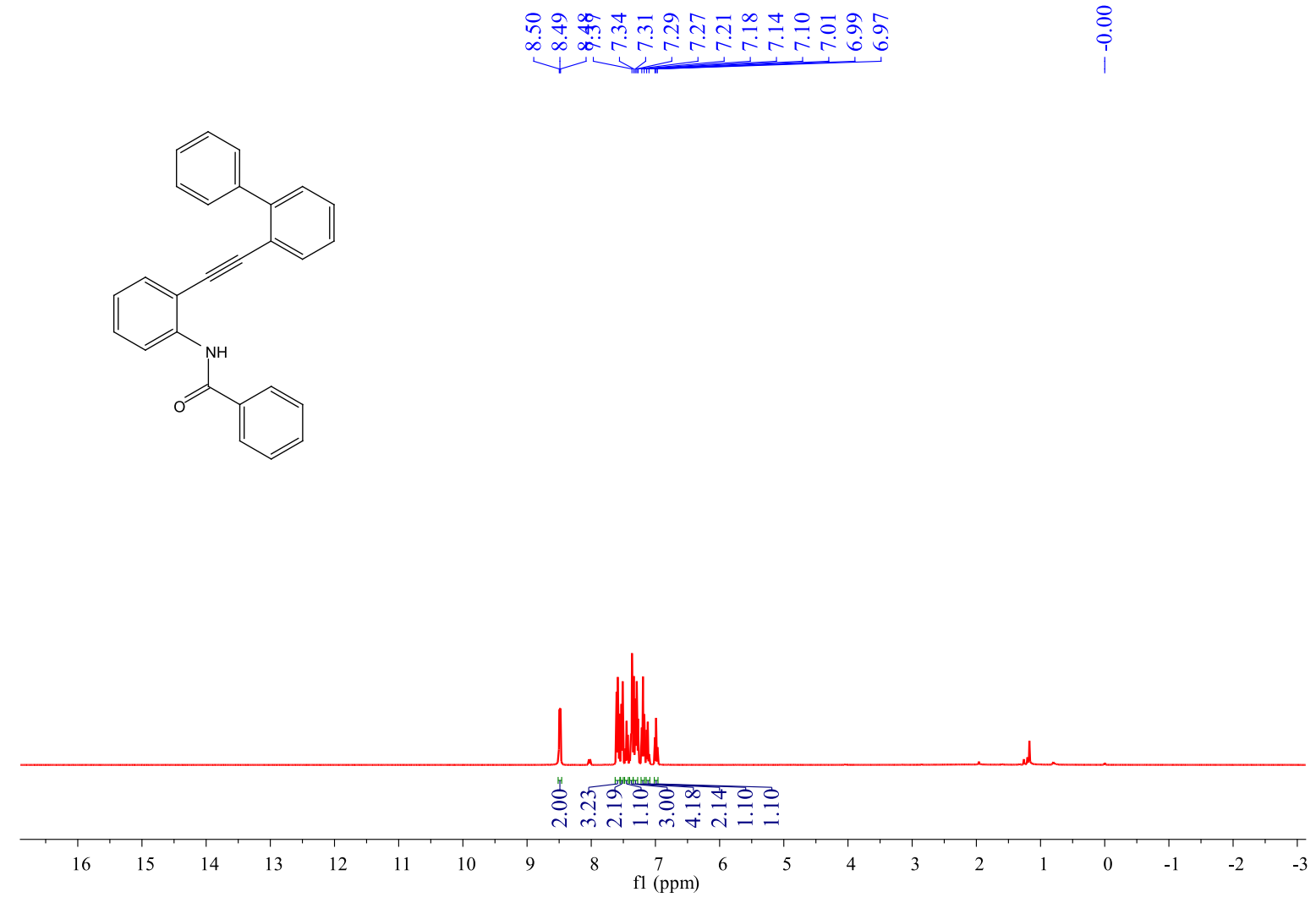

${ }^{1}$ H NMR Spectrum of Compound 1a
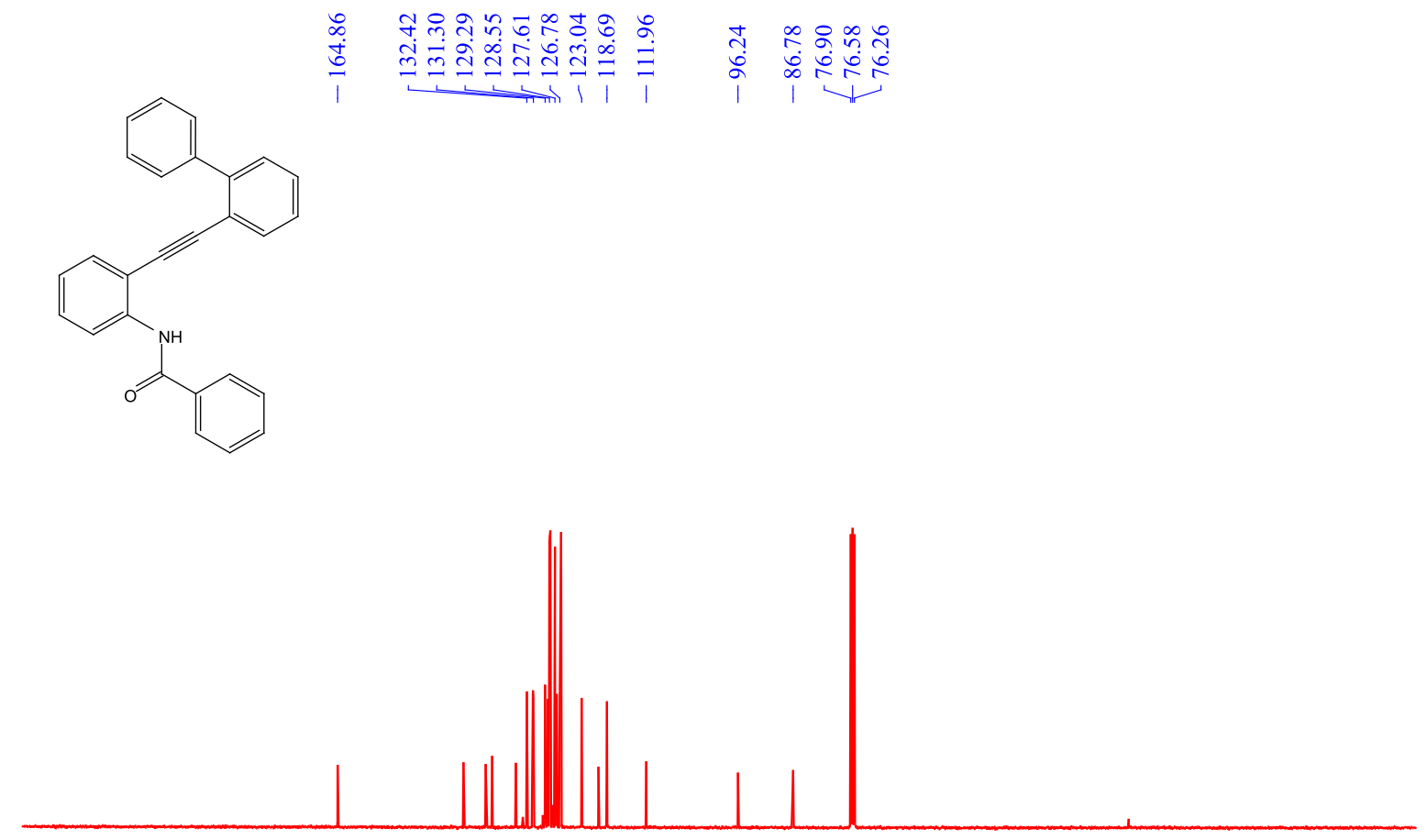

$\begin{array}{lllllllllllllllllllllll}210 & 200 & 190 & 180 & 170 & 160 & 150 & 140 & 130 & 120 & 110 & \begin{array}{l}100 \\ \mathrm{fl}(\mathrm{ppm})\end{array} & 90 & 80 & 70 & 60 & 50 & 40 & 30 & 20 & 10 & 0 & -10\end{array}$

${ }^{13} \mathrm{C}$ NMR Spectrum of Compound 1a 

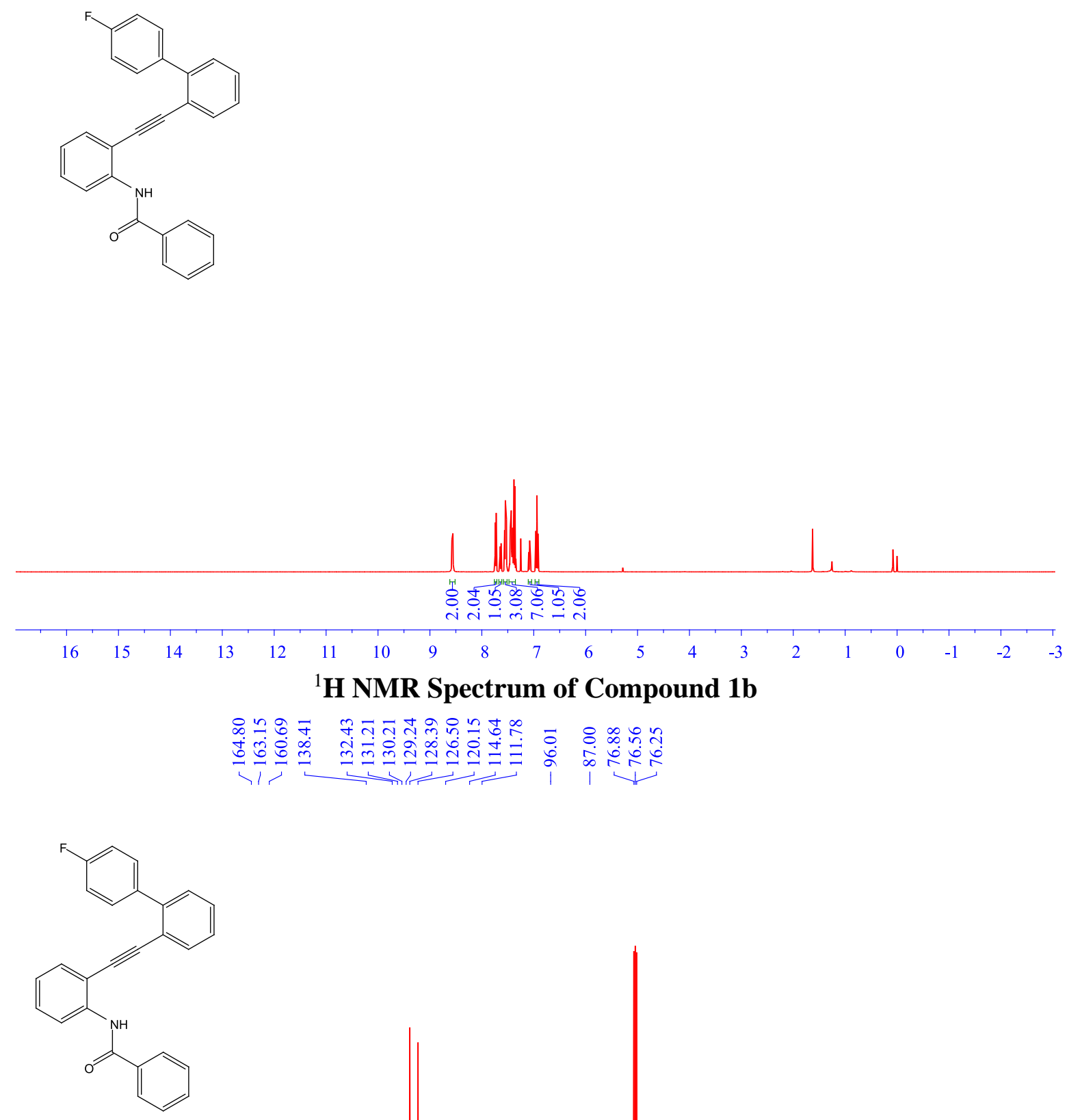

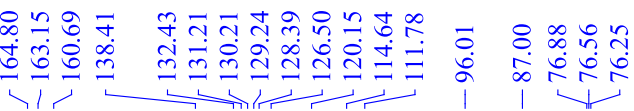

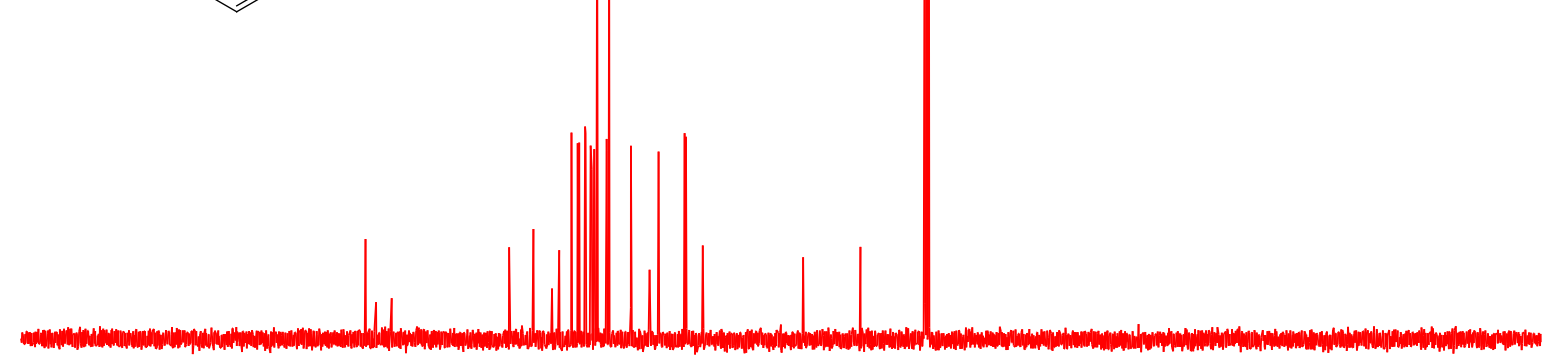

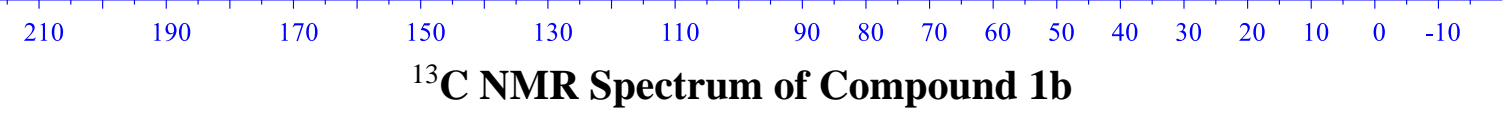




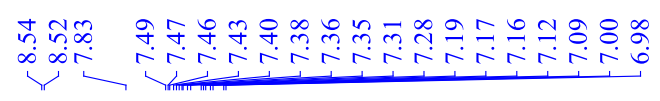

$\stackrel{8}{i}$
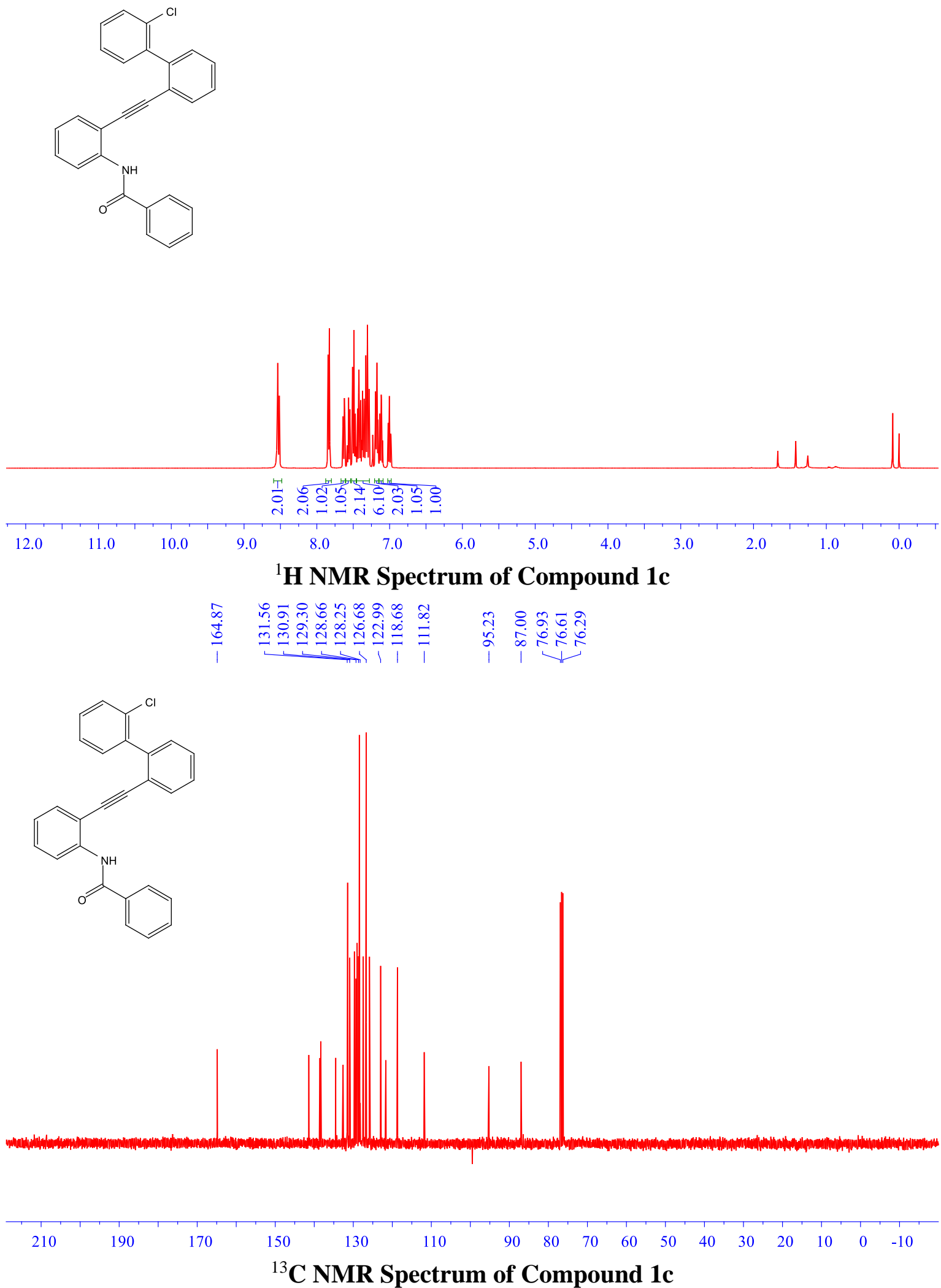

S45 
<smiles>O=C(Nc1ccccc1C#Cc1ccccc1-c1ccc(Cl)cc1)c1ccccc1</smiles>

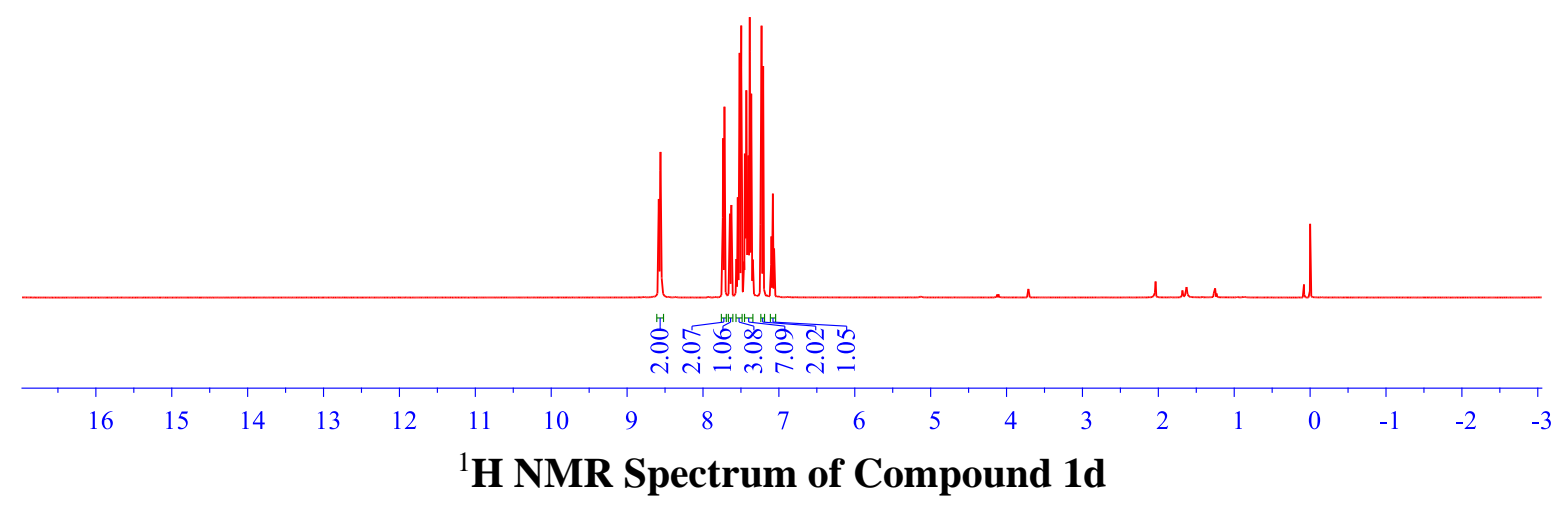

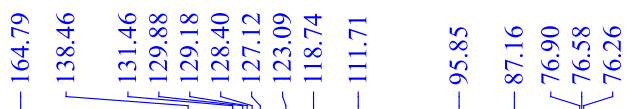

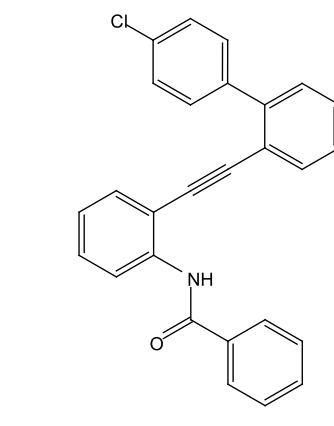

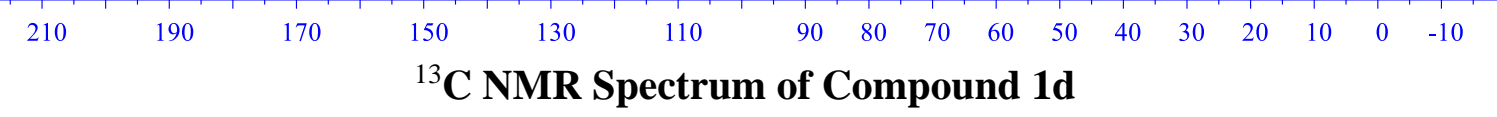




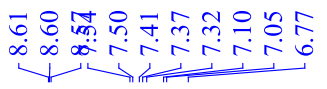

$\underset{i}{\stackrel{7}{i}}$

$\stackrel{8}{8}$
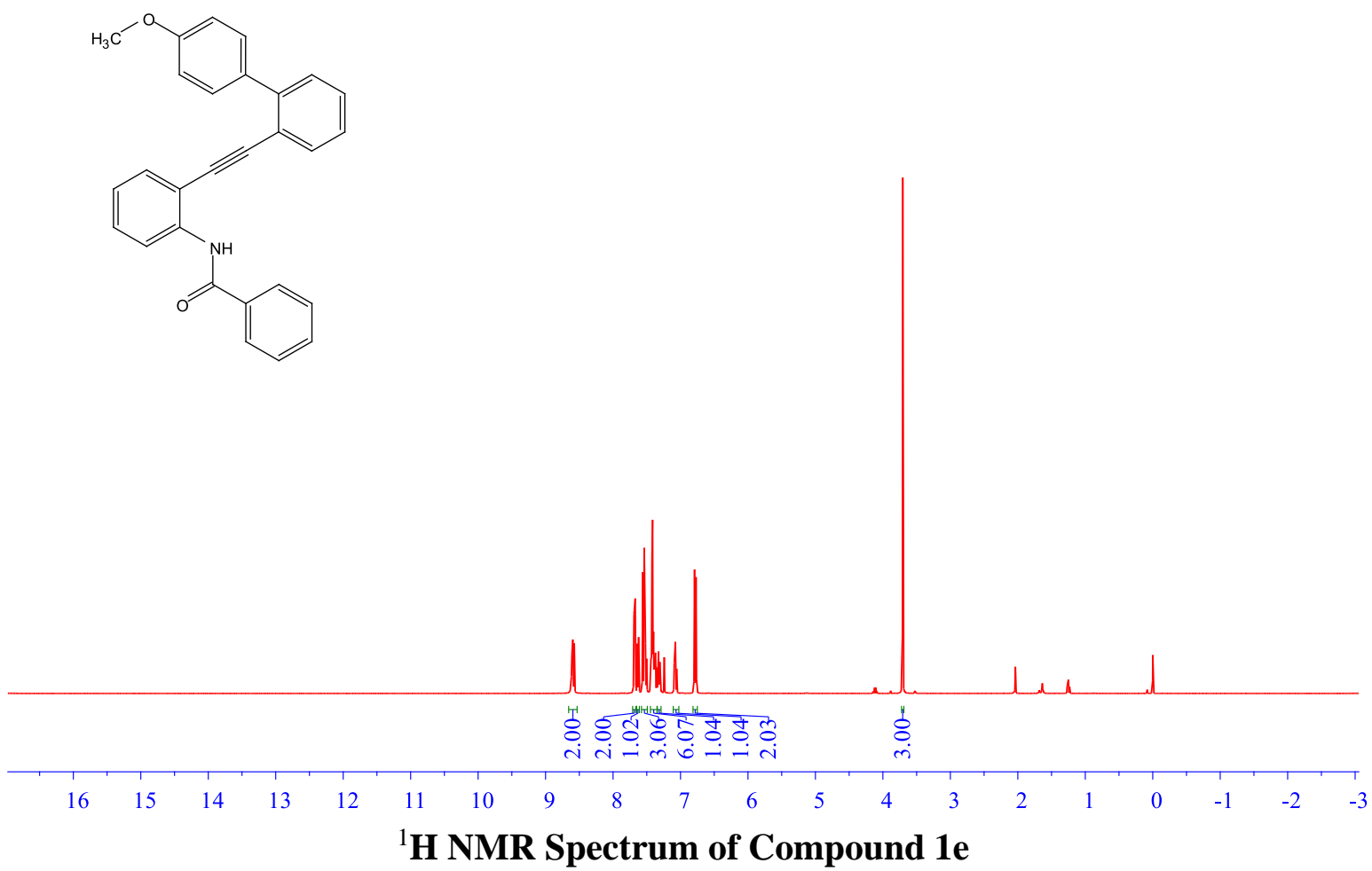

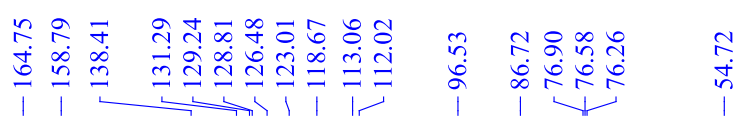
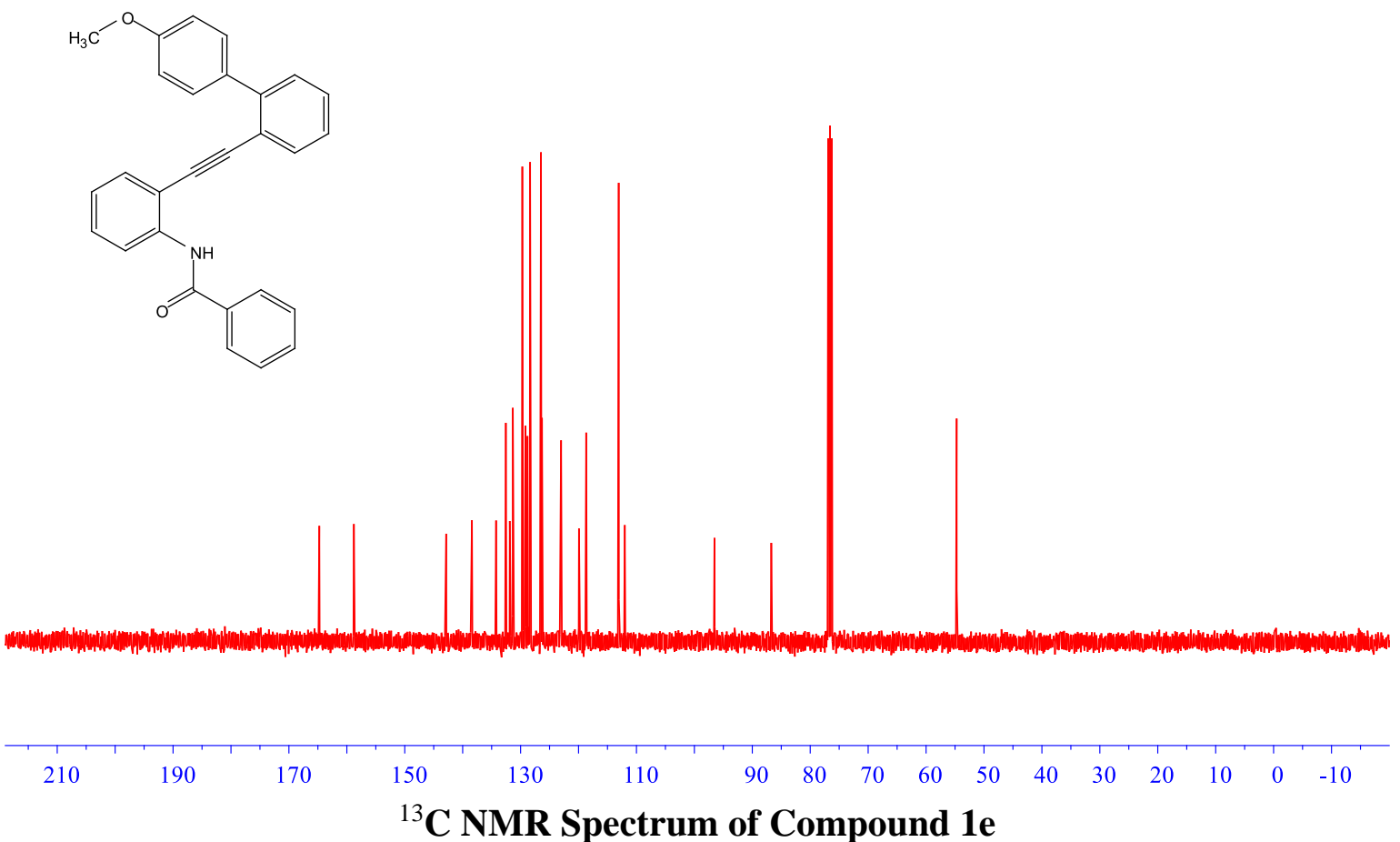

S47 


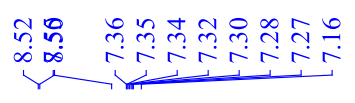

$\begin{array}{ll} \pm & 8 \\ i & 0 \\ i & i\end{array}$
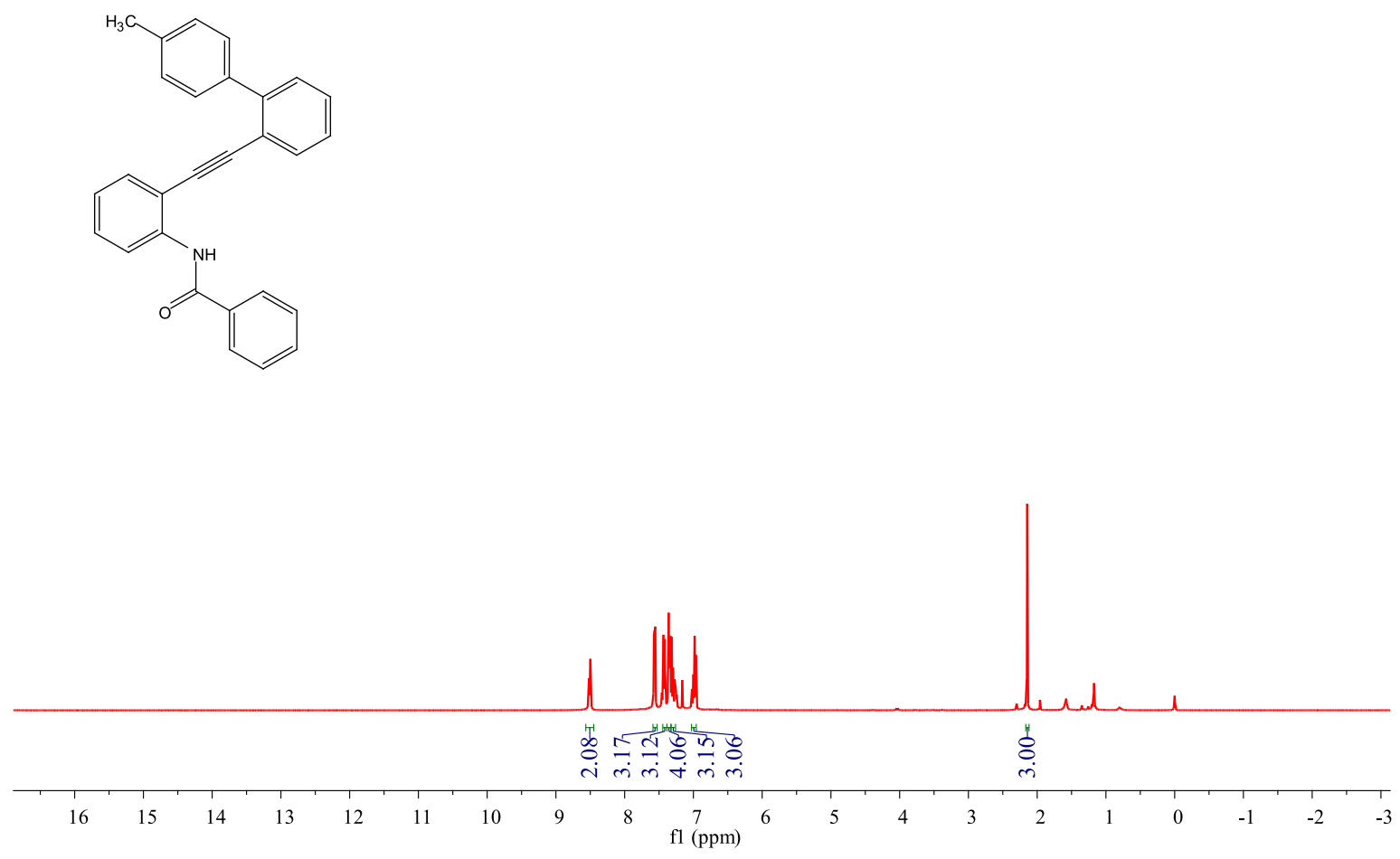

${ }^{1}$ H NMR Spectrum of Compound if

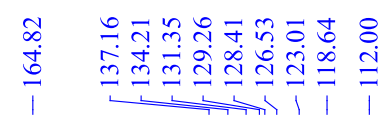

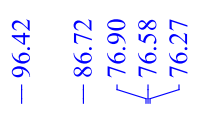

$\underset{\substack{0 \\ 0}}{1}$
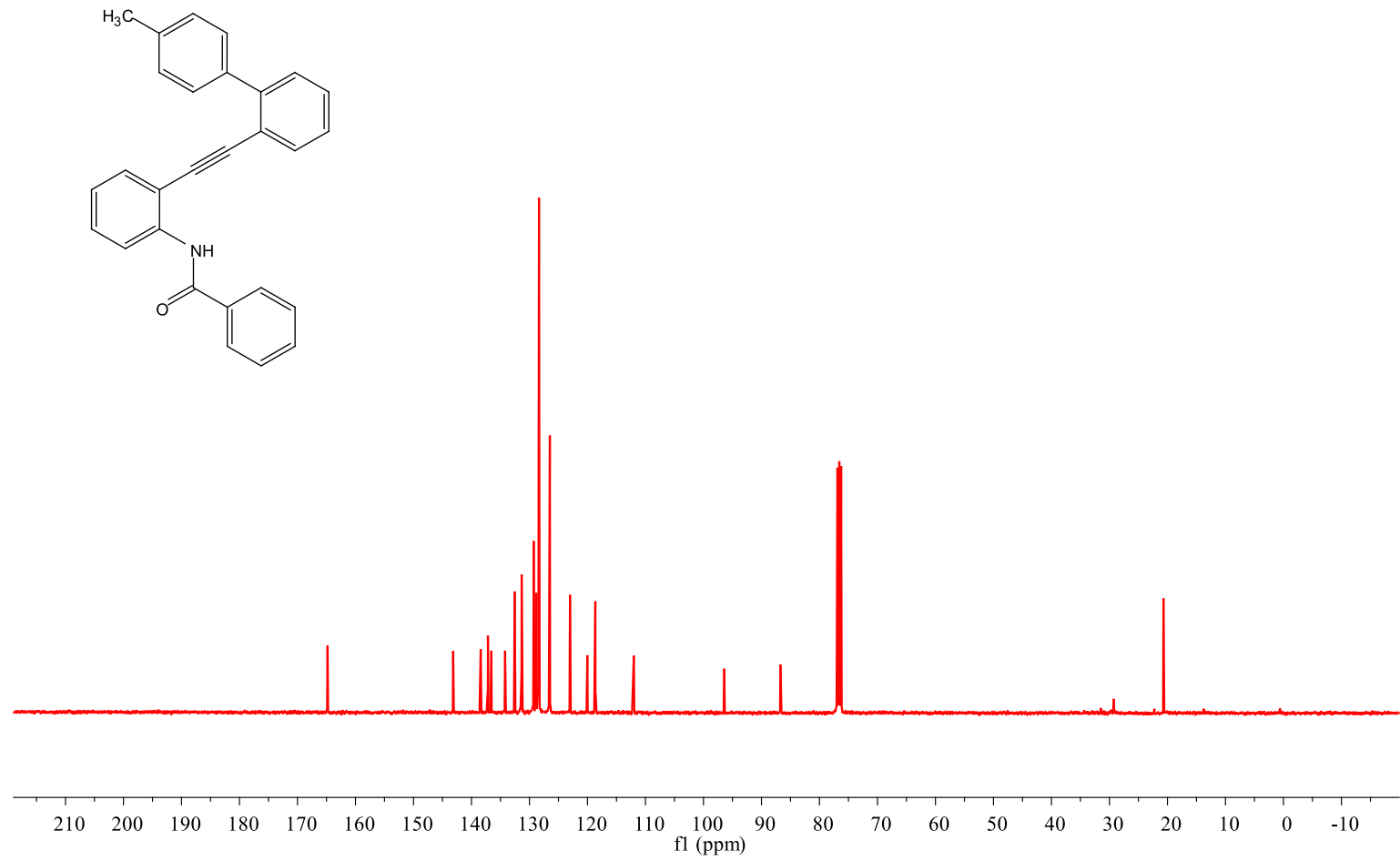

${ }^{13} \mathrm{C}$ NMR Spectrum of Compound if

S48 
$\underbrace{\infty}$

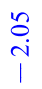

$\stackrel{8}{0}$
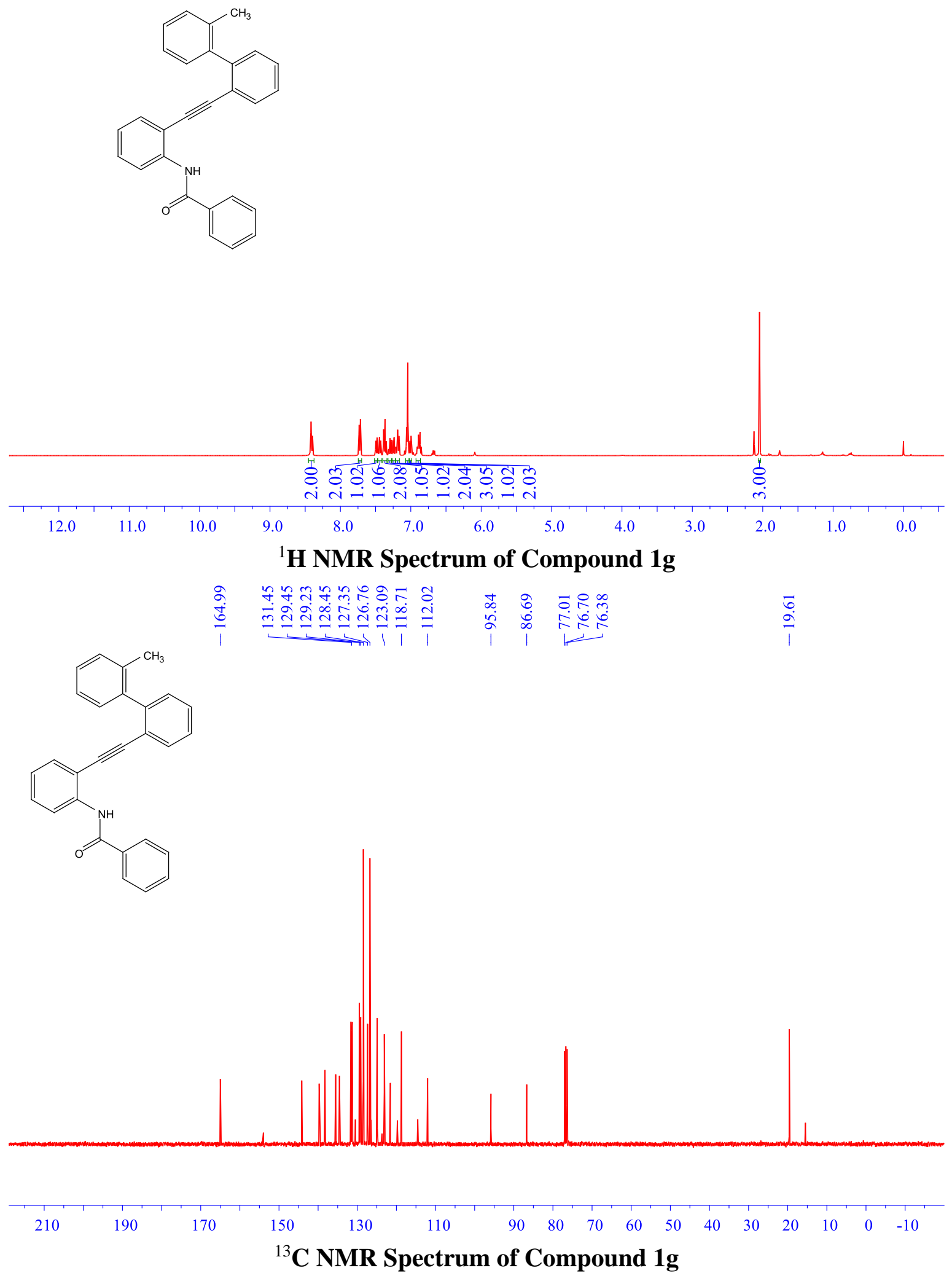

S49 


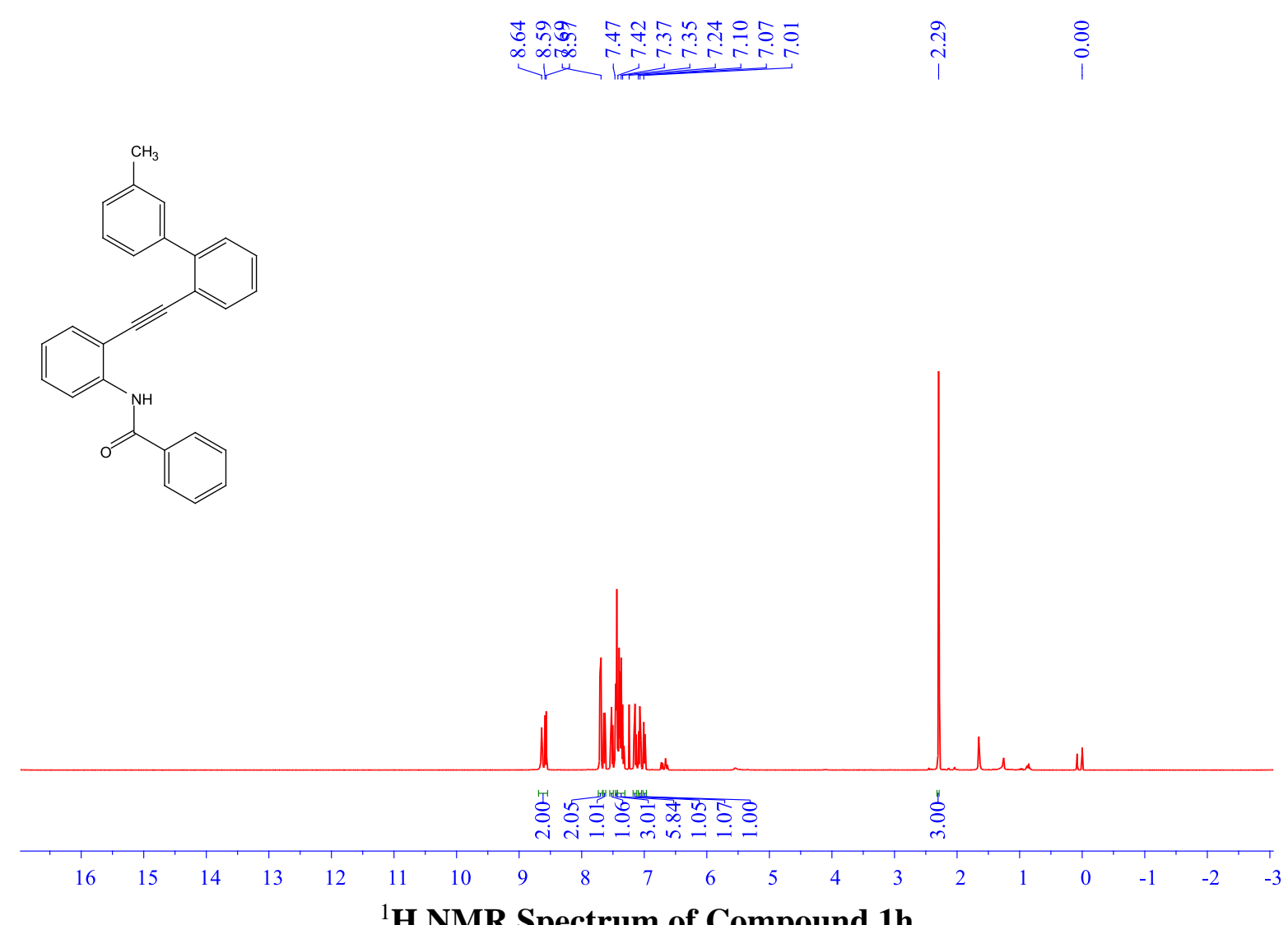

${ }^{1}$ H NMR Spectrum of Compound $1 \mathrm{~h}$

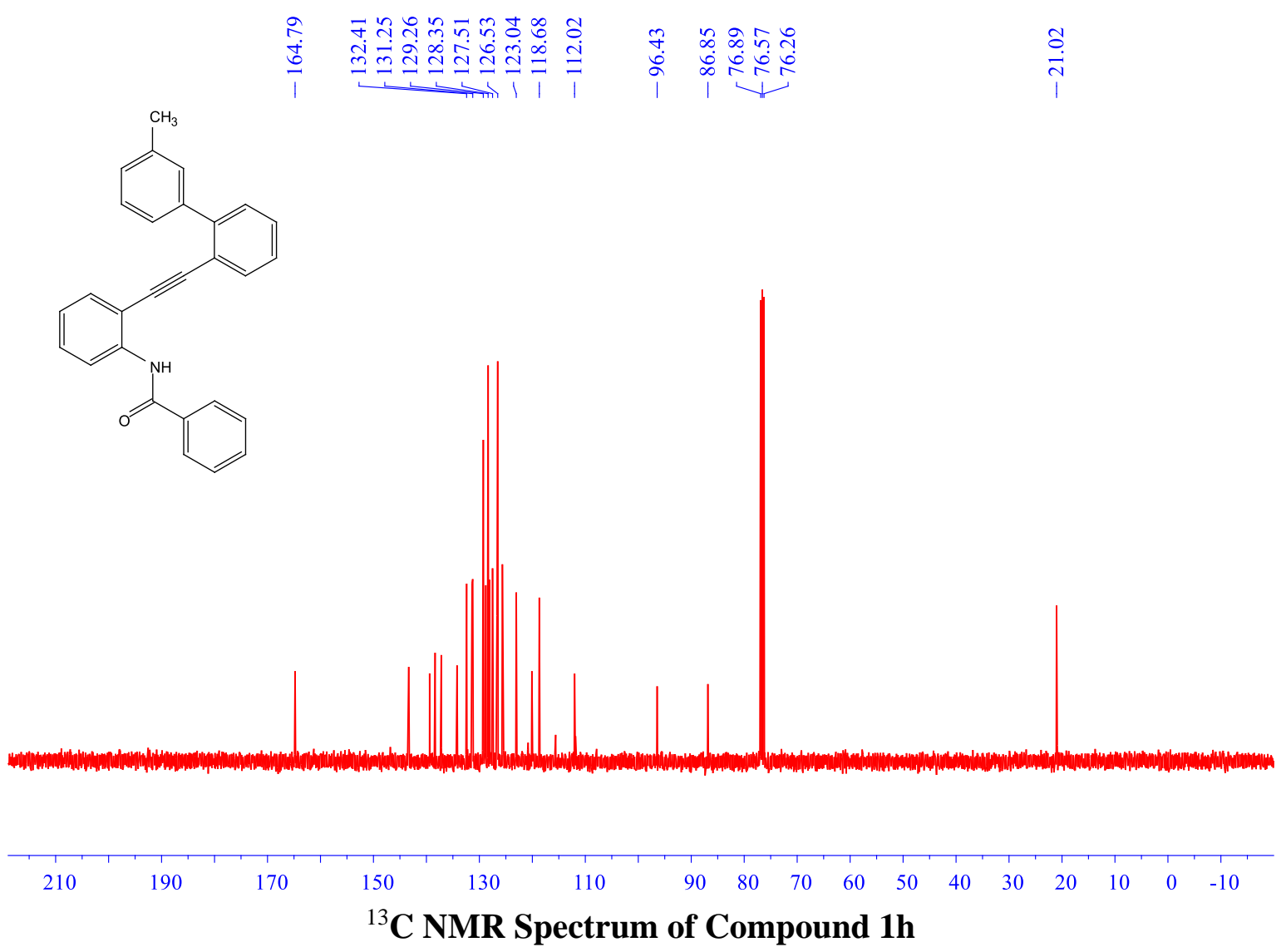



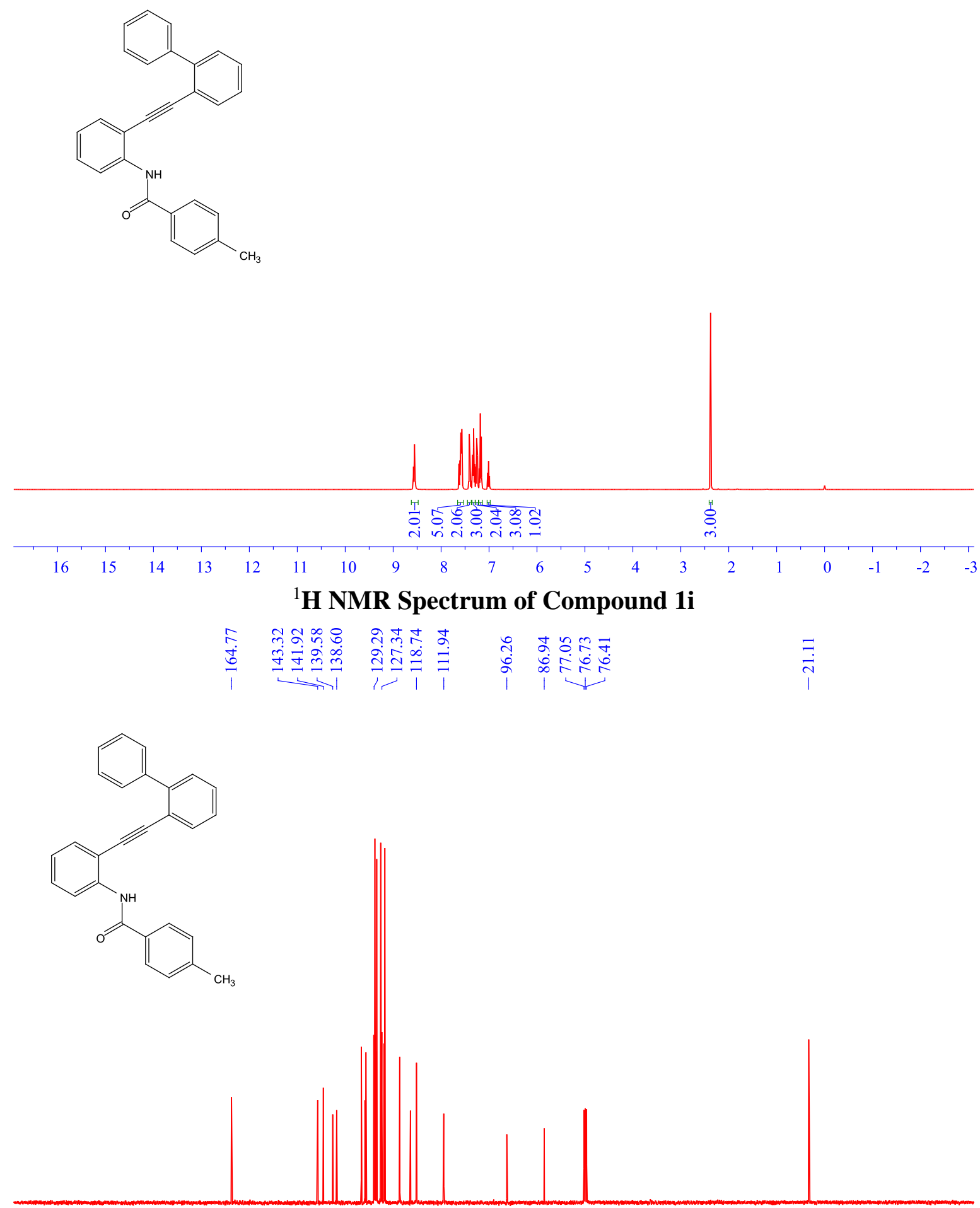

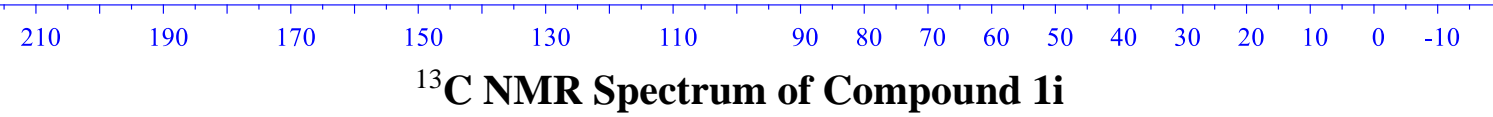



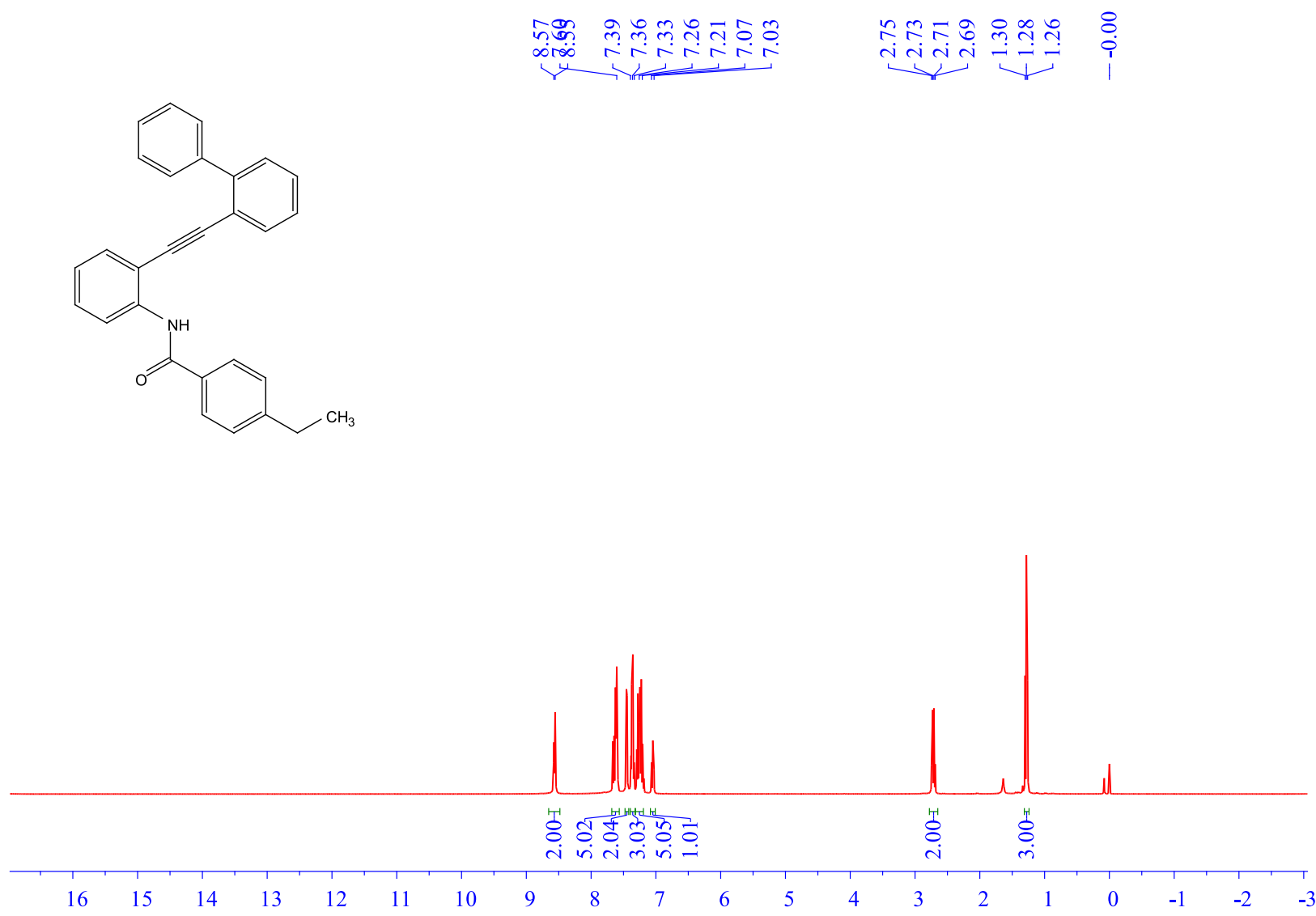

${ }^{1}$ H NMR Spectrum of Compound $1 \mathbf{j}$

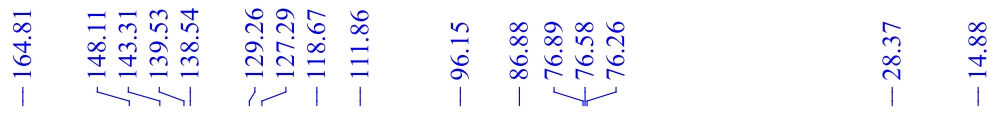

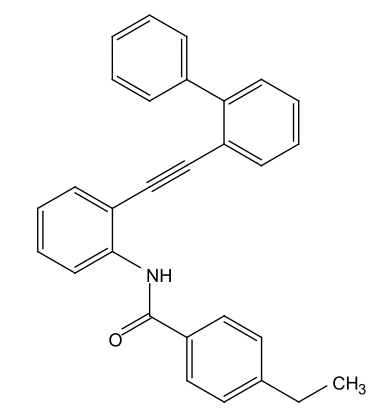

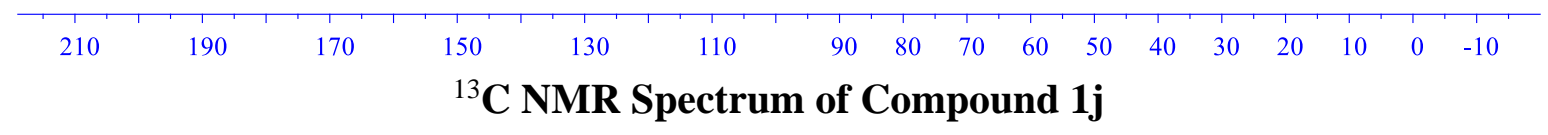



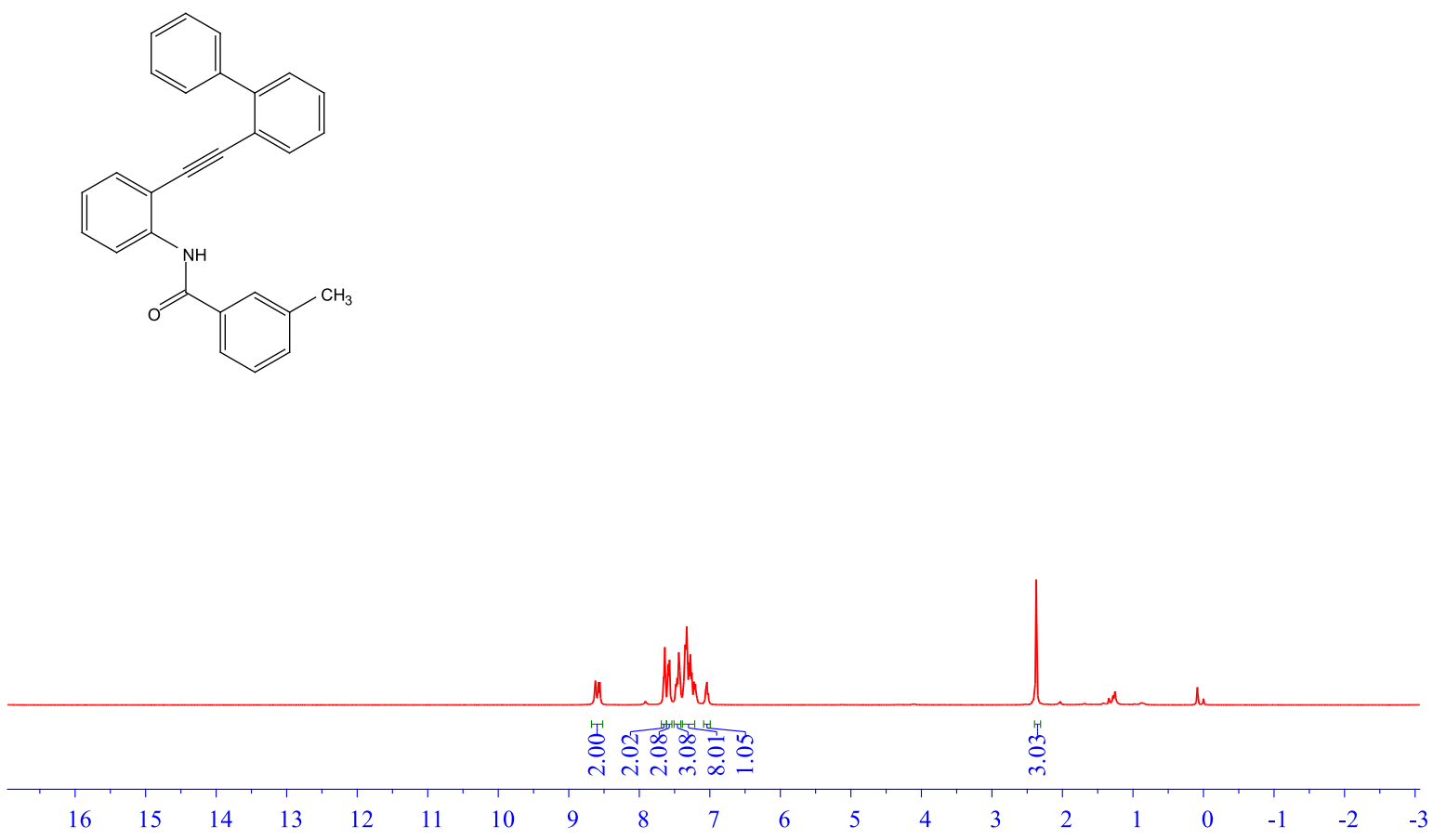

${ }^{1} \mathrm{H}$ NMR Spectrum of Compound 1k
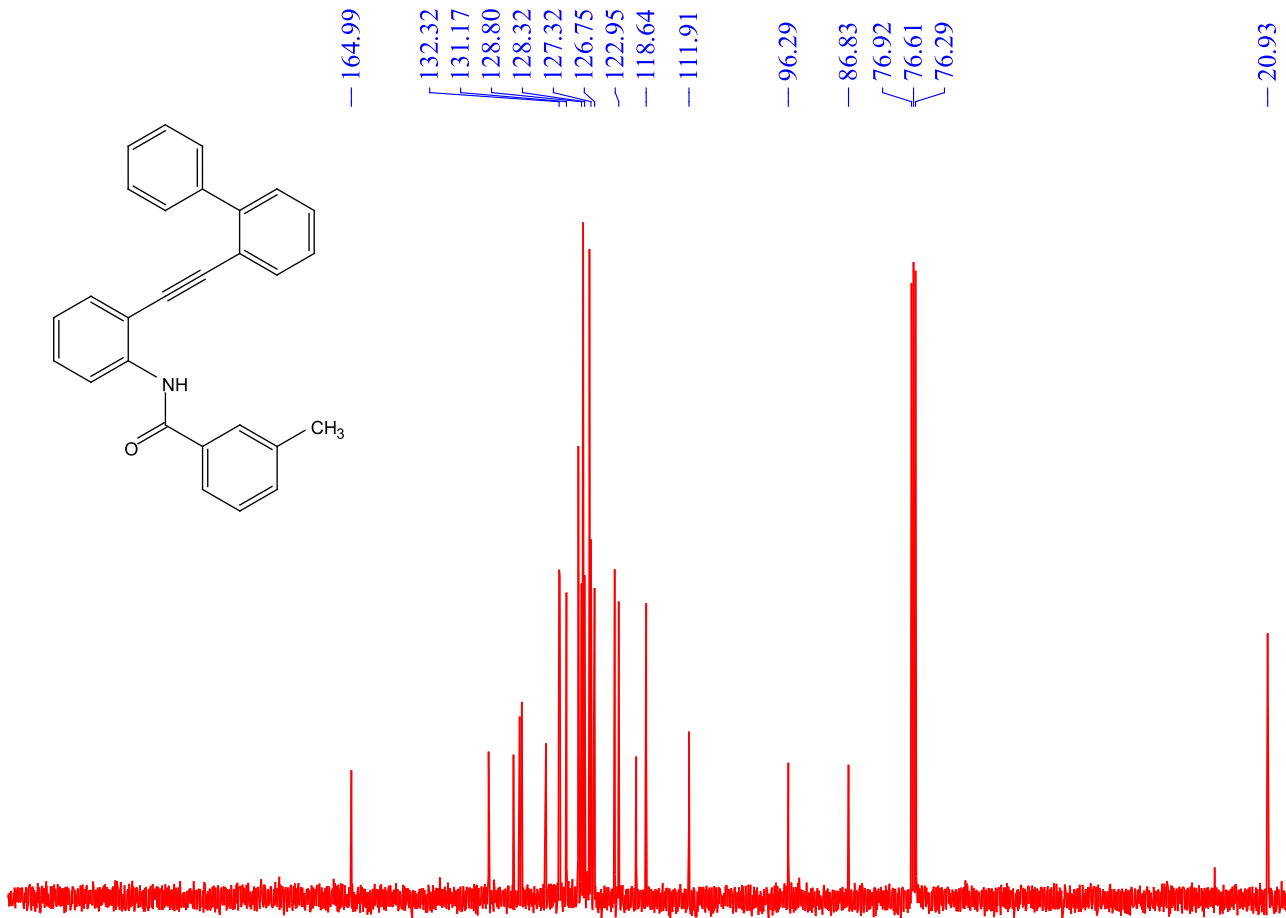

210

190

170

150

130

110

$\begin{array}{lll}90 & 80 & 70\end{array}$

$60 \quad 50$

in

${ }^{13} \mathrm{C}$ NMR Spectrum of Compound $1 \mathrm{k}$ 

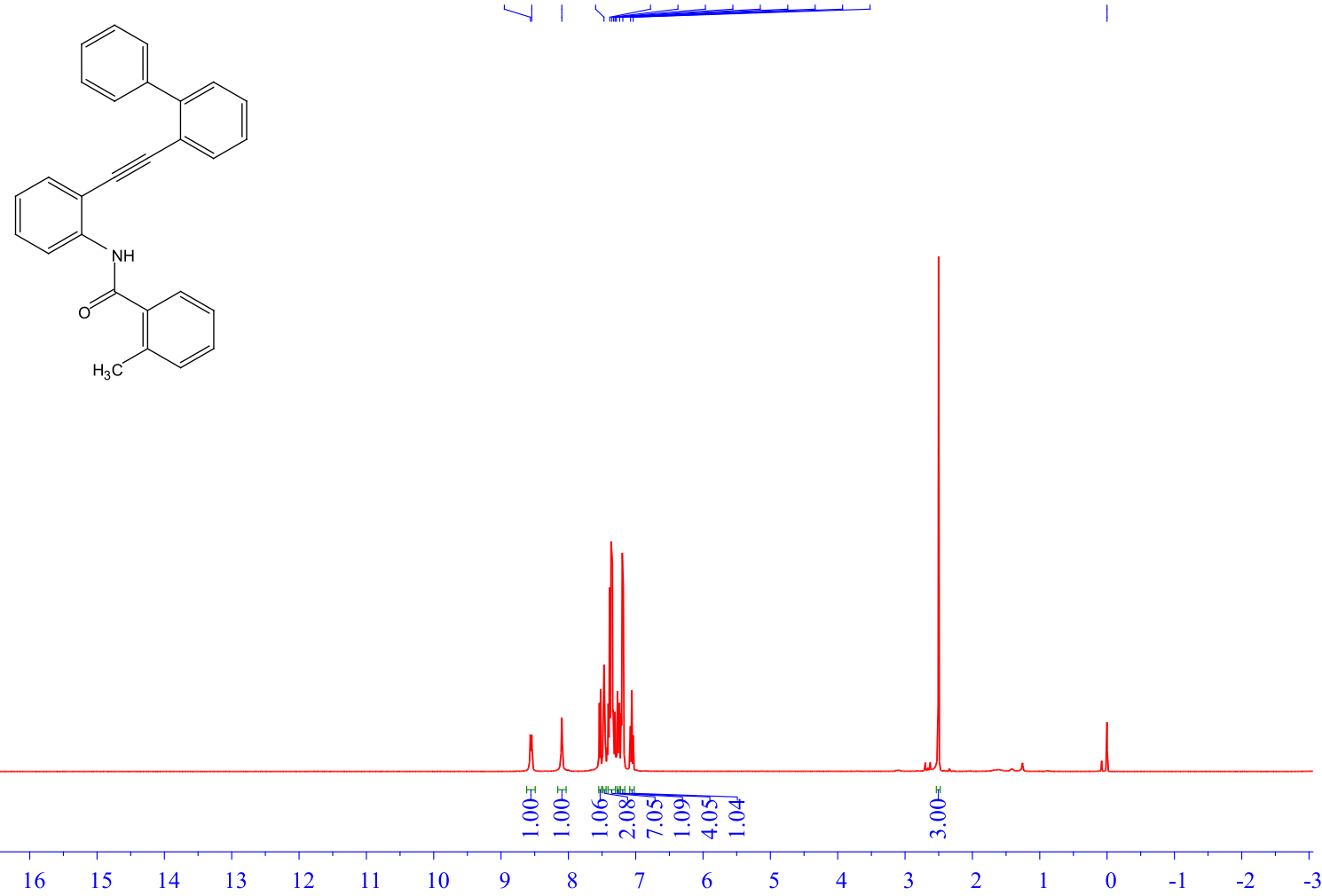

${ }^{1} \mathrm{H}$ NMR Spectrum of Compound 11

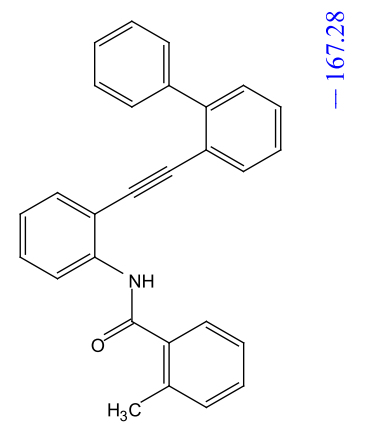

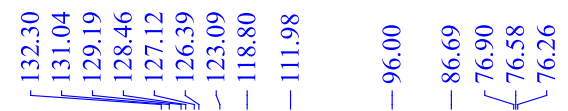

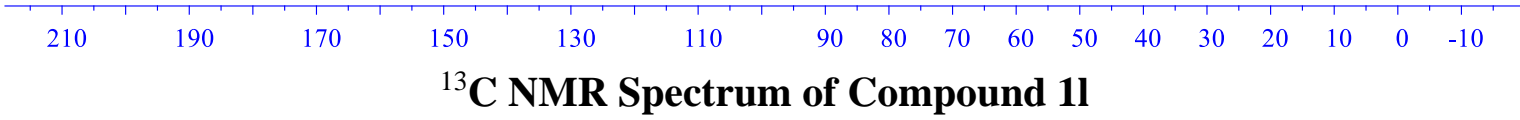




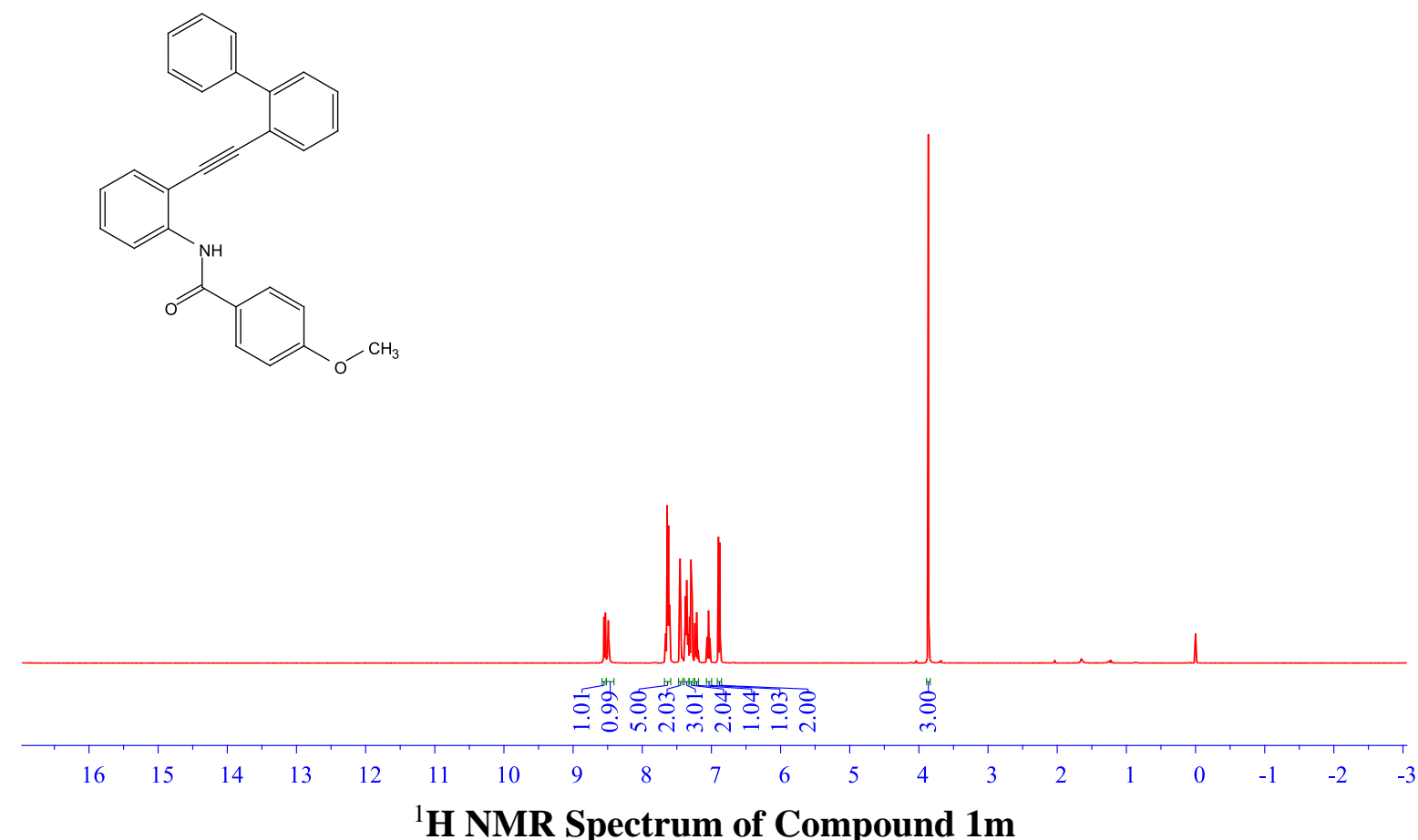

${ }^{1} \mathrm{H}$ NMR Spectrum of Compound $1 \mathrm{~m}$

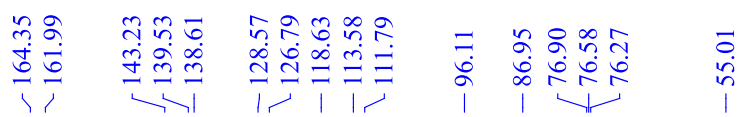
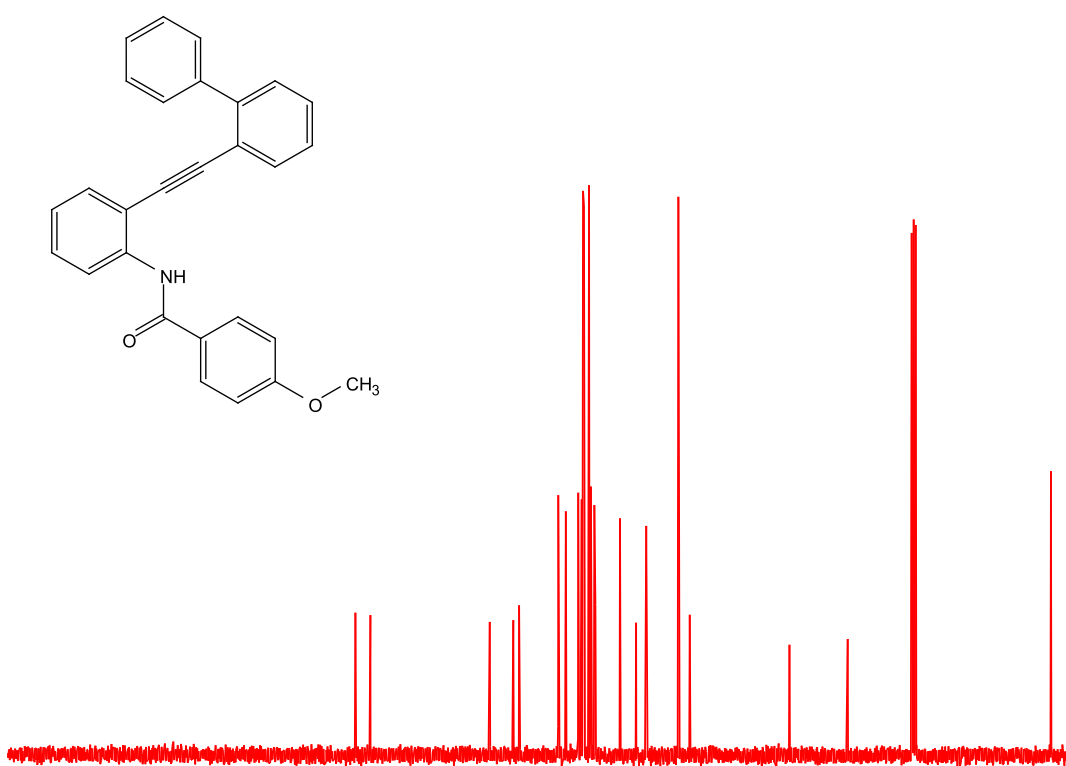

\begin{tabular}{lllllllllllllllllll}
\hline 210 & 190 & 170 & 150 & 130 & 110 & 90 & 80 & 70 & 60 & 50 & 40 & 30 & 20 & 10 & 0 & -10 \\
& & & & & &
\end{tabular}




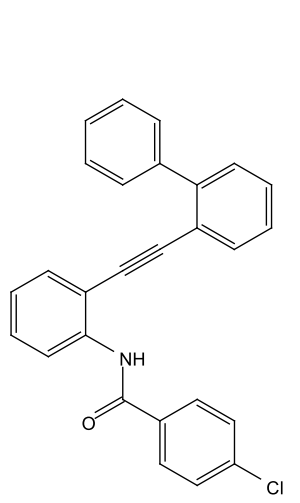

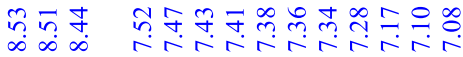

$\stackrel{8}{8}$

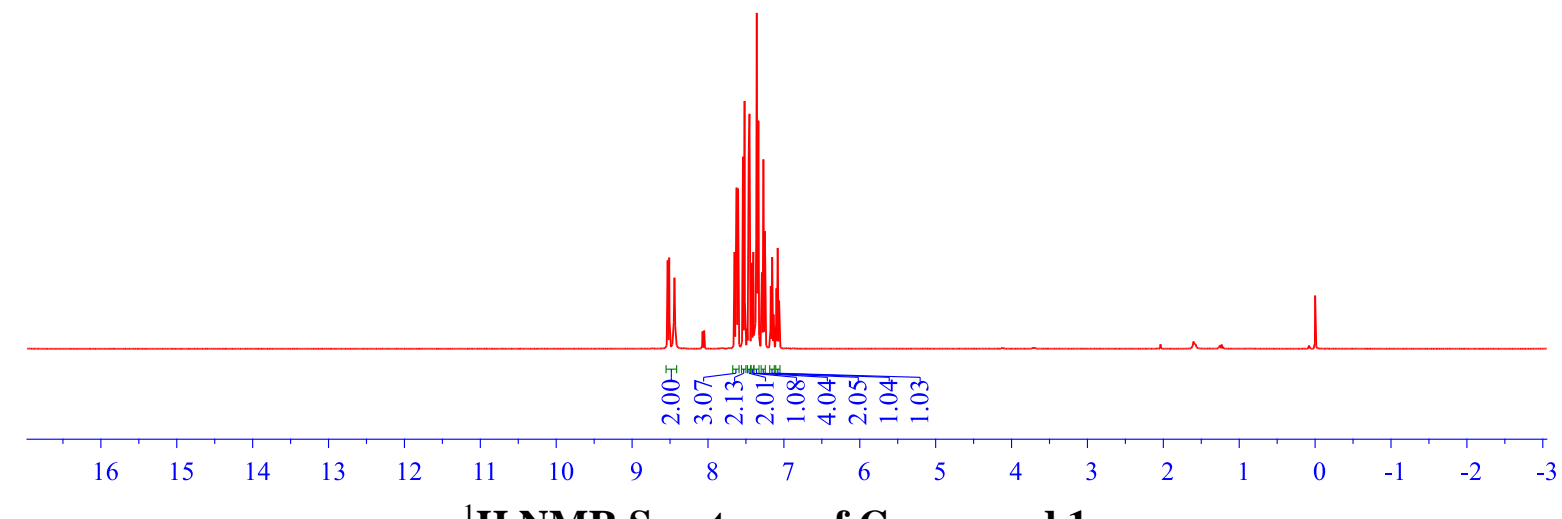

${ }^{1} \mathrm{H}$ NMR Spectrum of Compound 1n

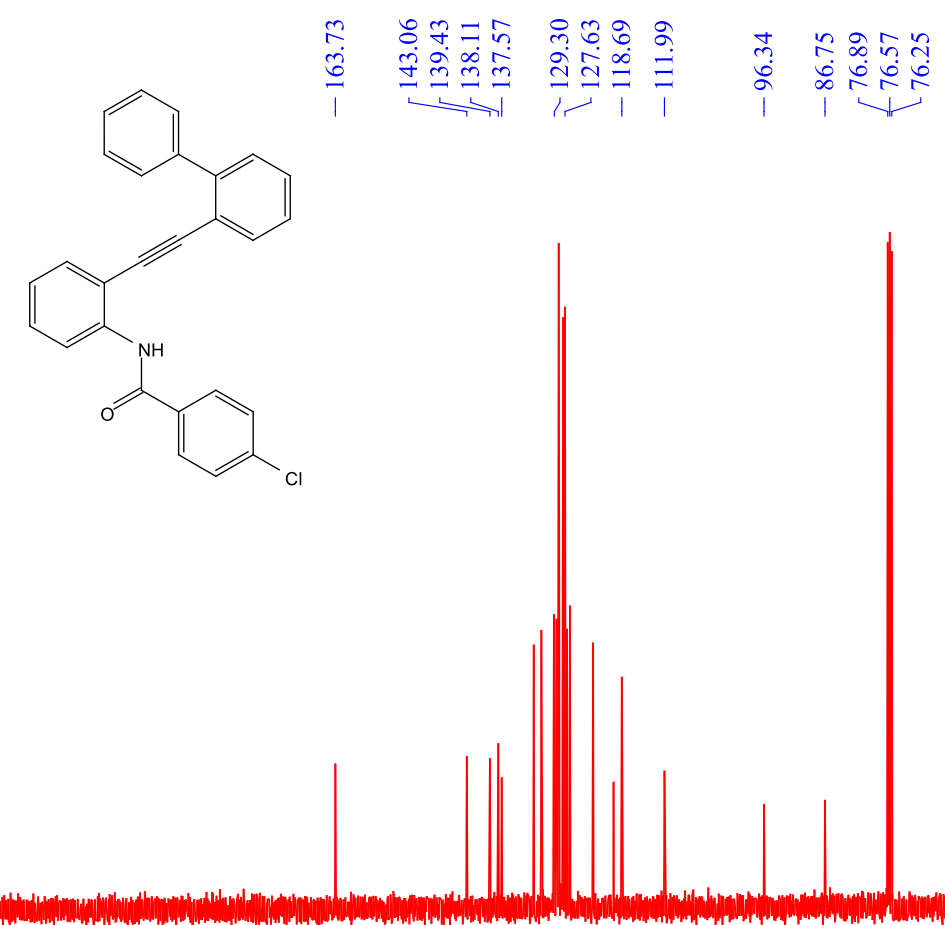

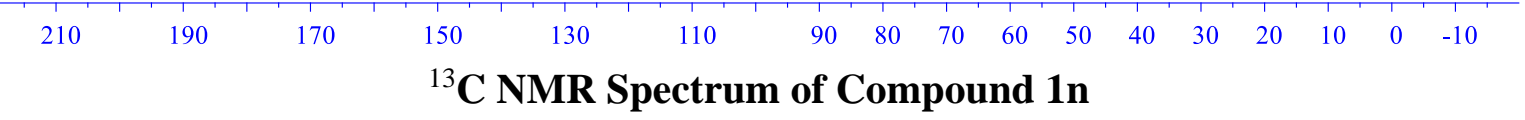




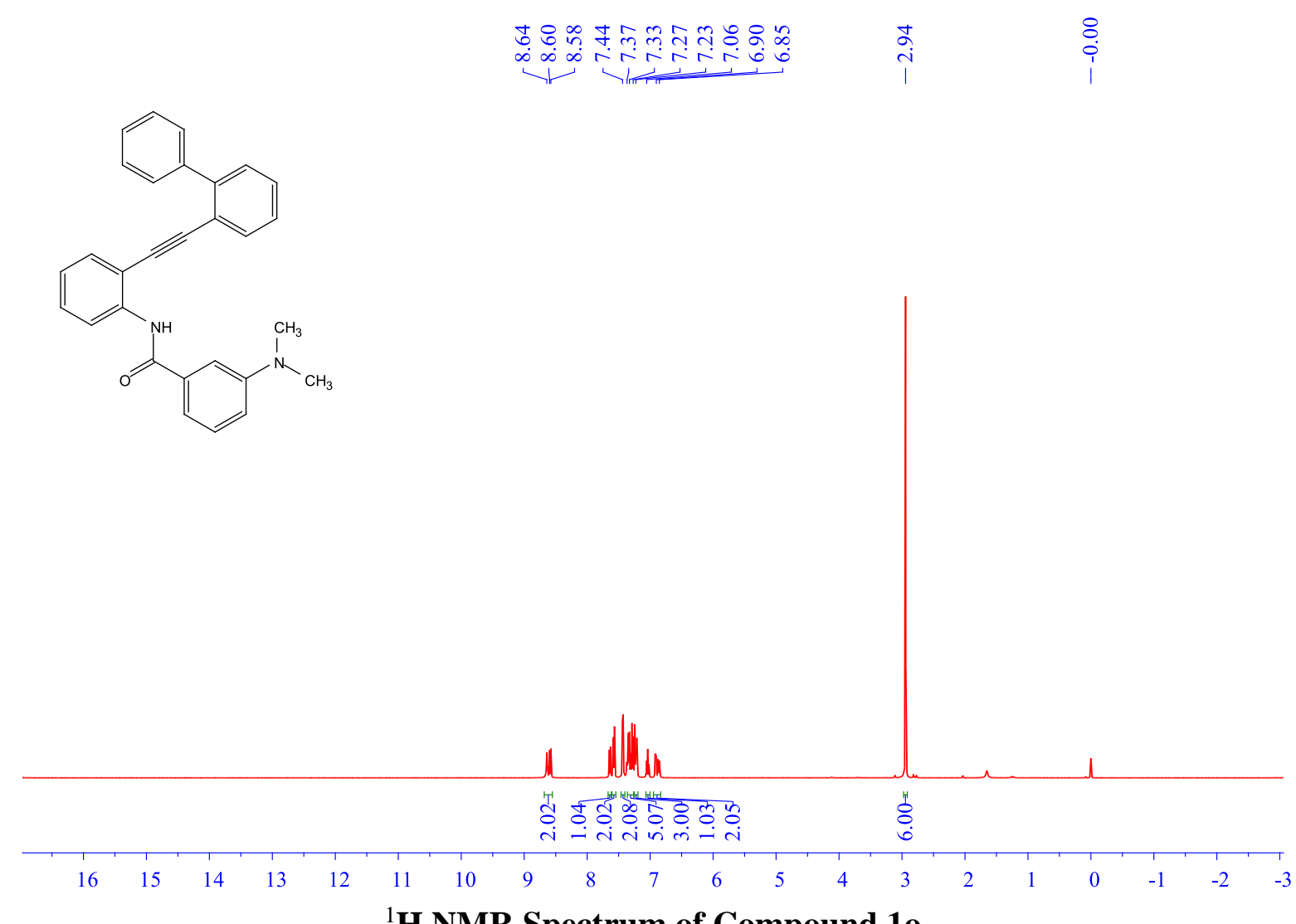

${ }^{1}$ H NMR Spectrum of Compound 10

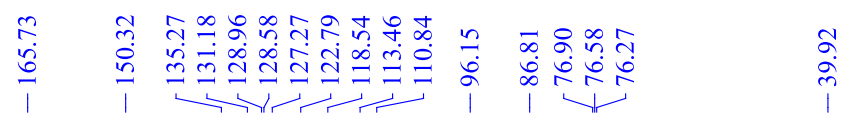

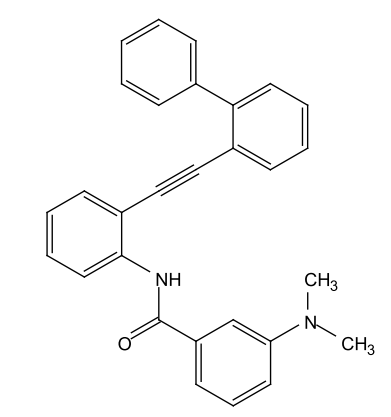

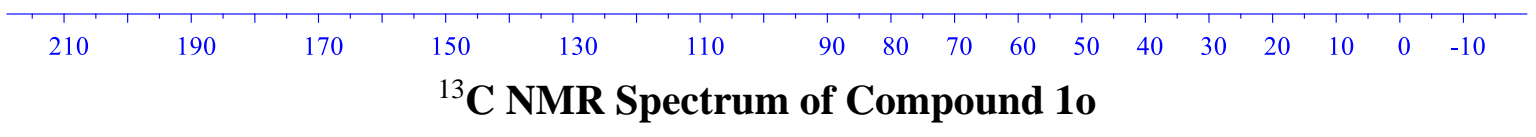



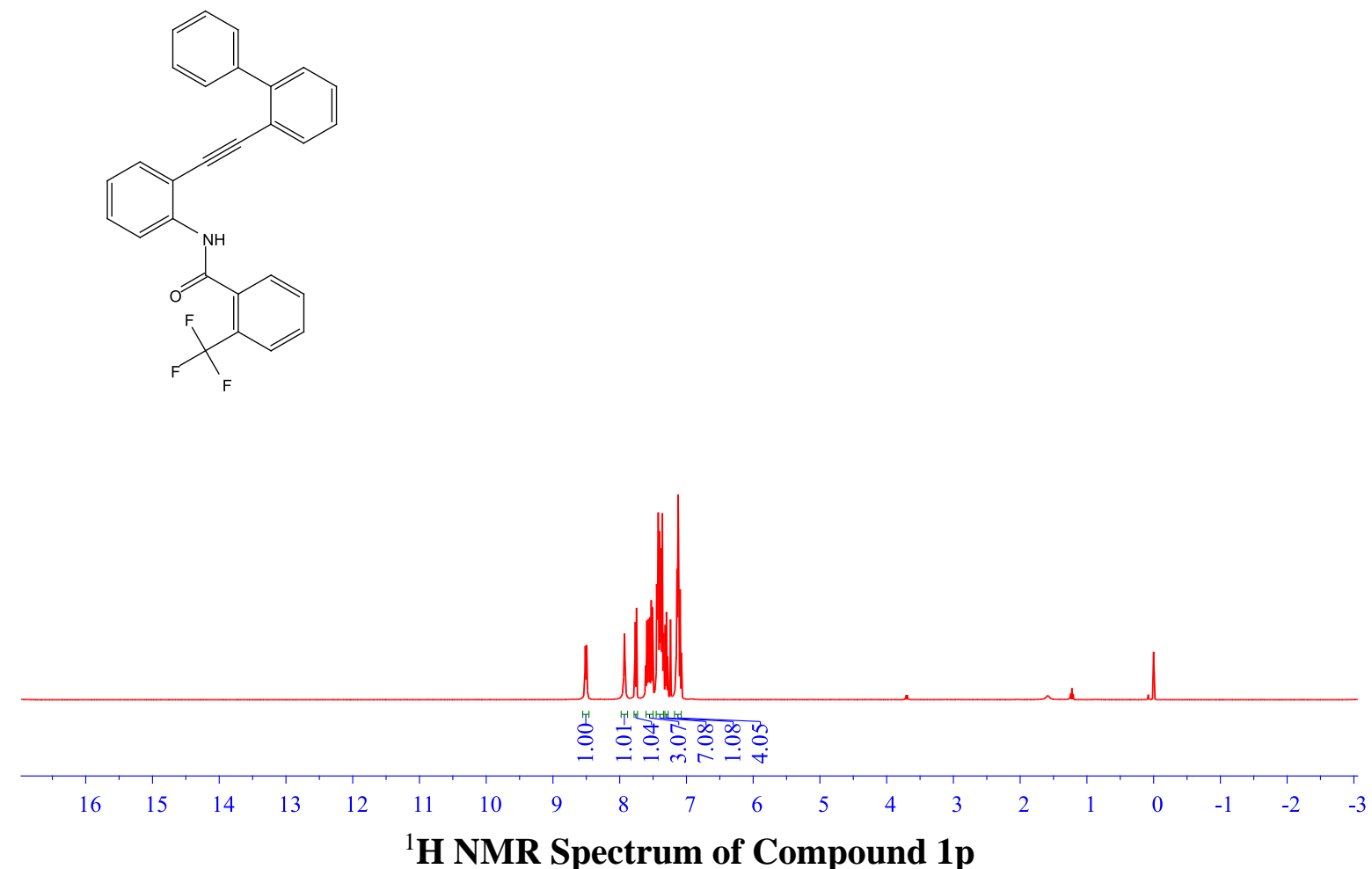

${ }^{1} \mathrm{H}$ NMR Spectrum of Compound 1p

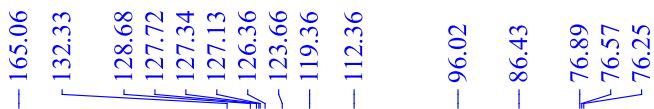
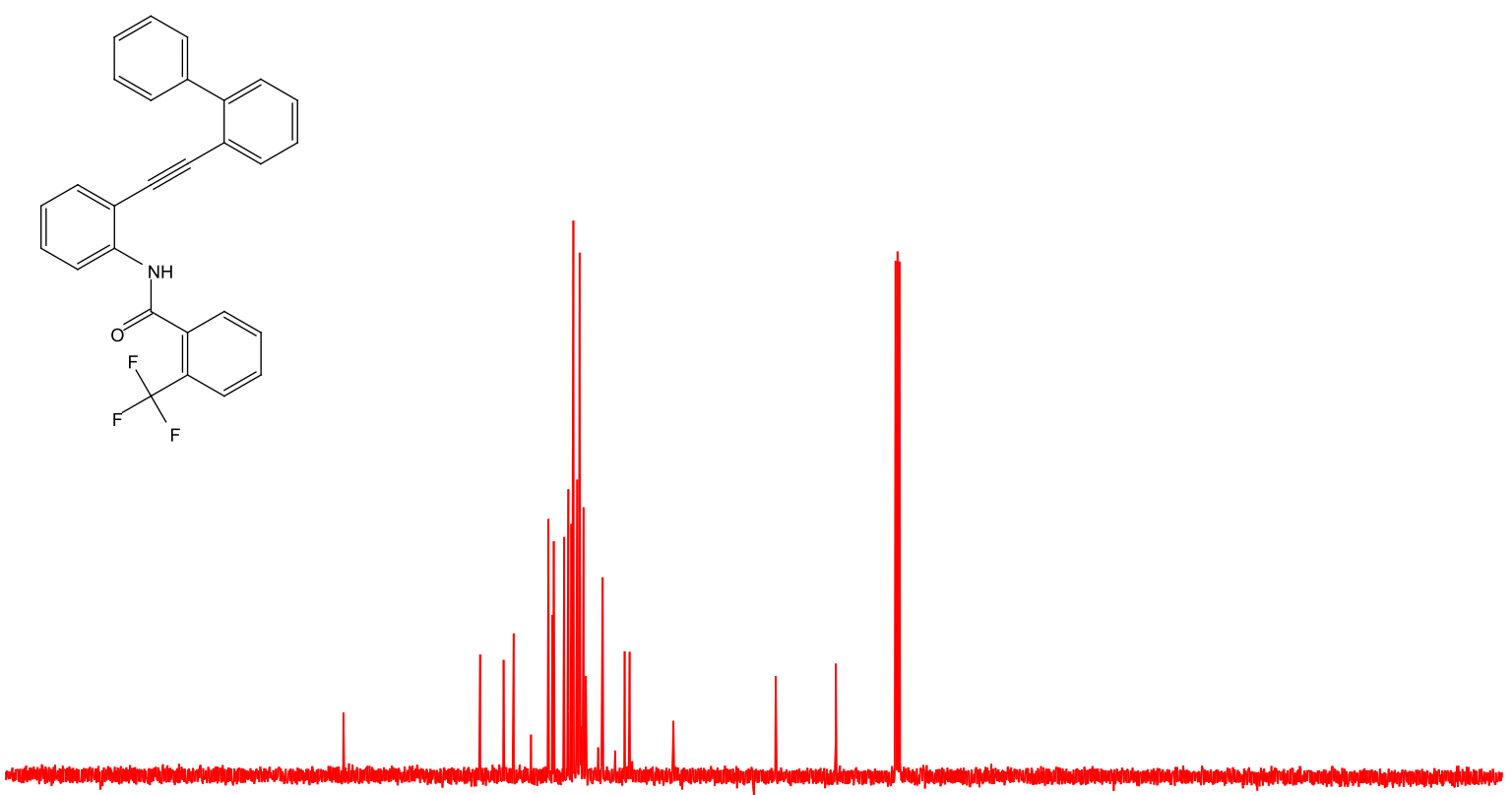

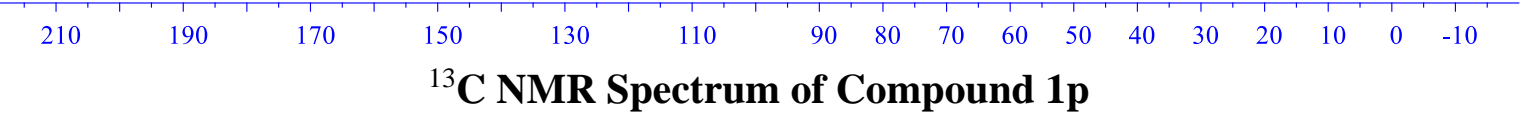



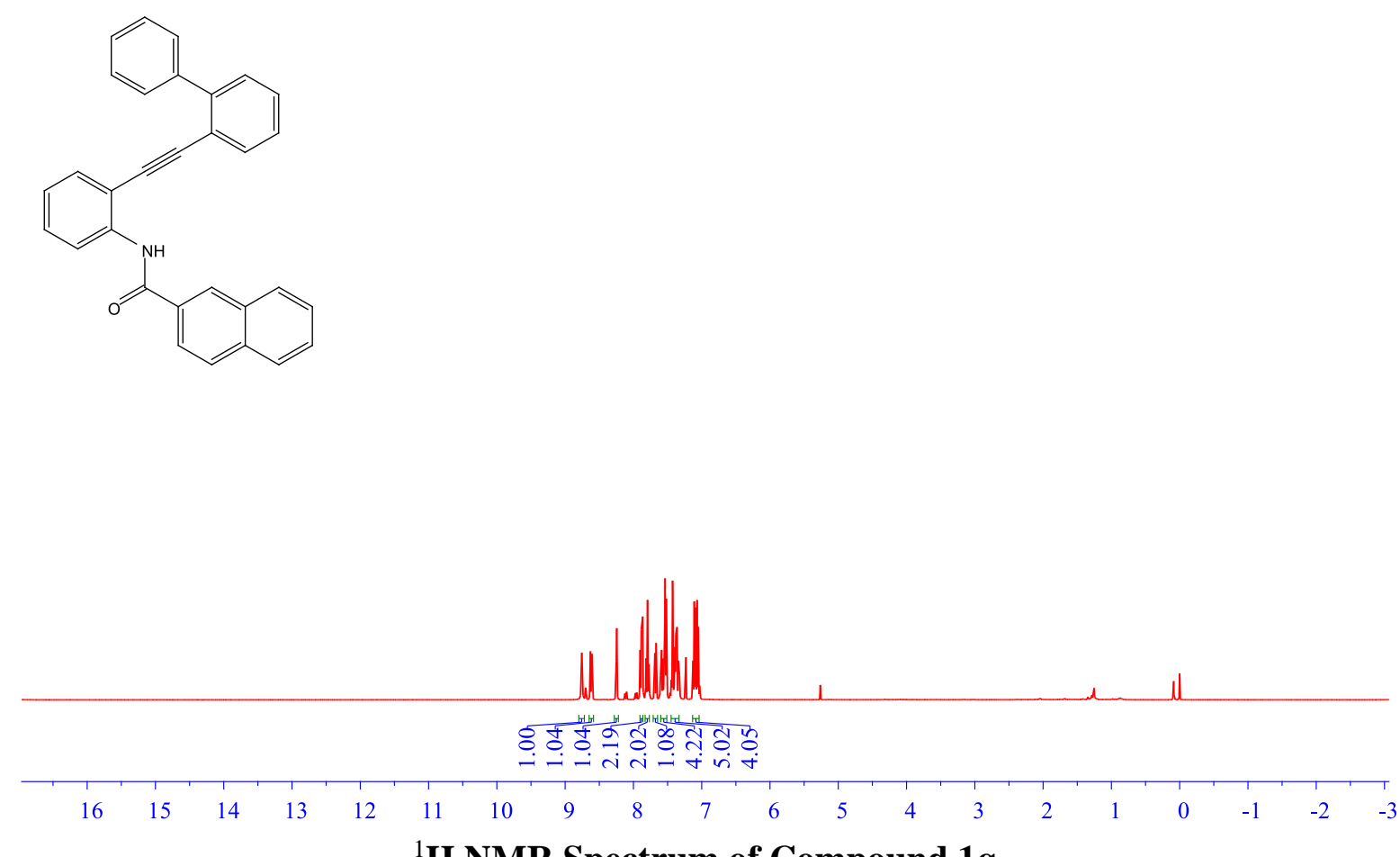

${ }^{1} \mathrm{H}$ NMR Spectrum of Compound $1 \mathrm{q}$

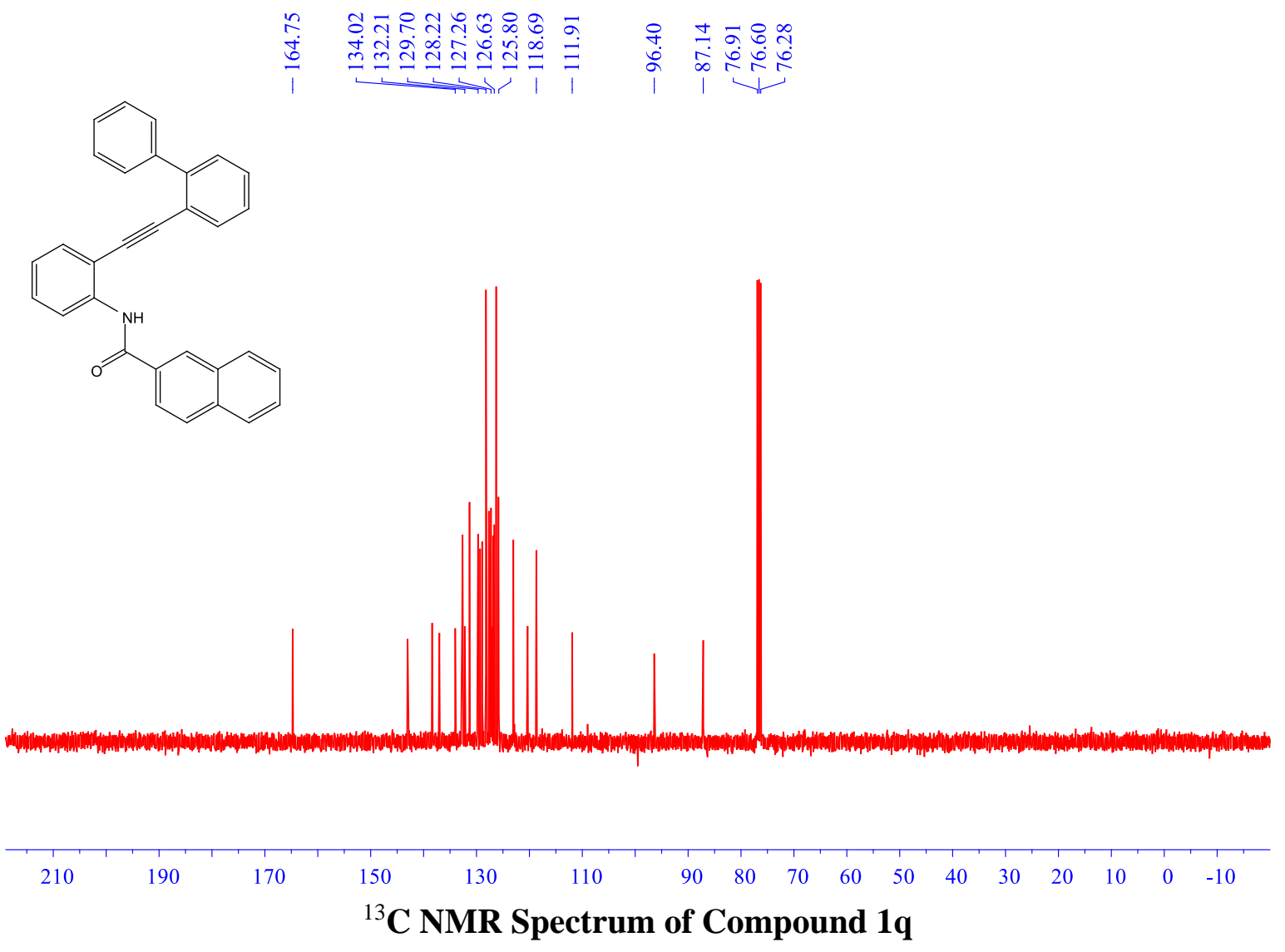



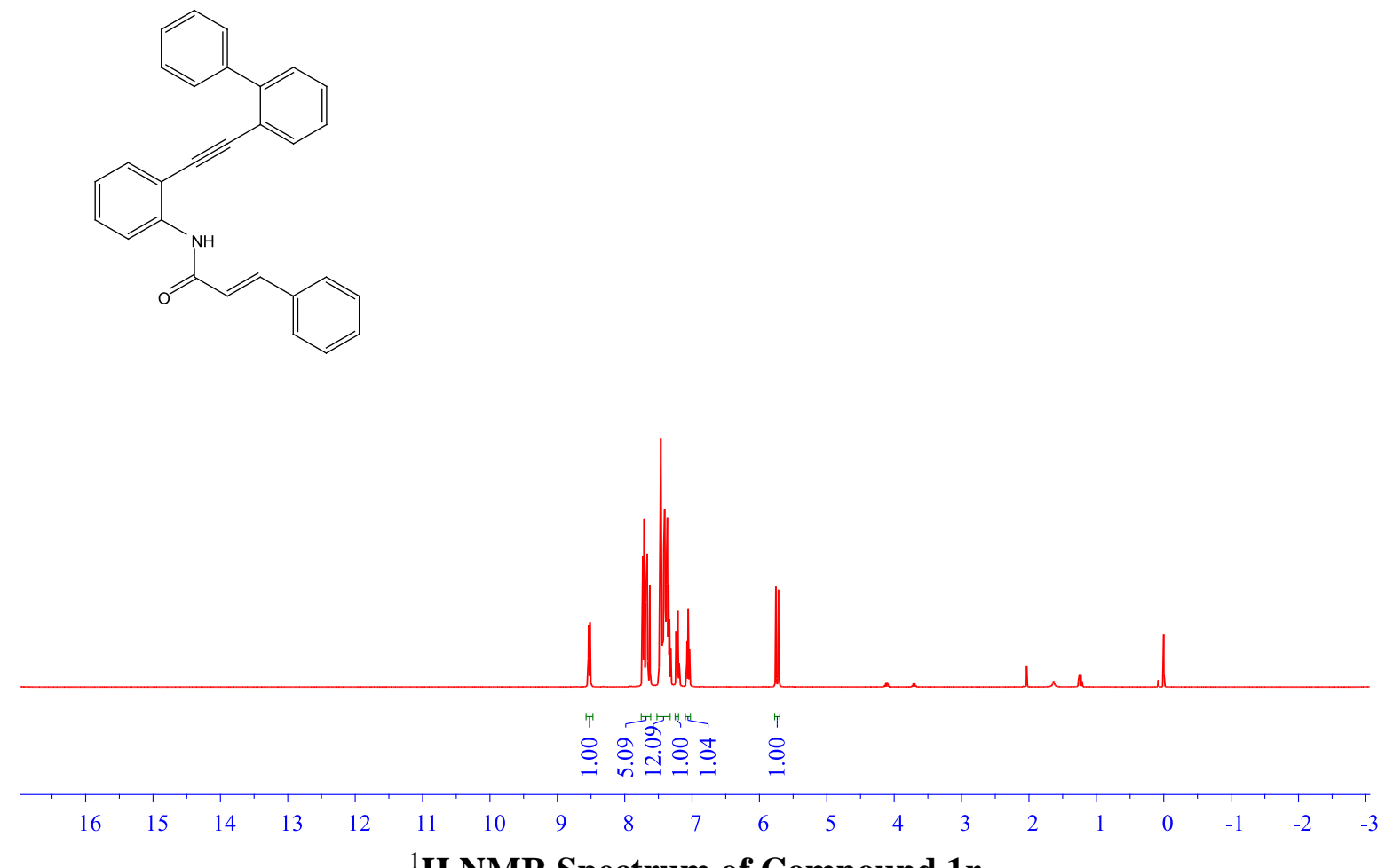

${ }^{1} \mathrm{H}$ NMR Spectrum of Compound $1 \mathrm{r}$

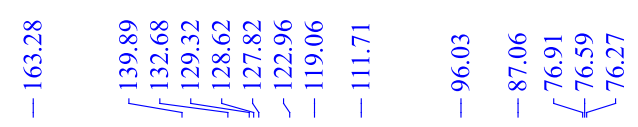<smiles>O=C(CCC1CCCCC1)CNC1CCCCC1Cc1ccccc1-c1ccccc1</smiles>

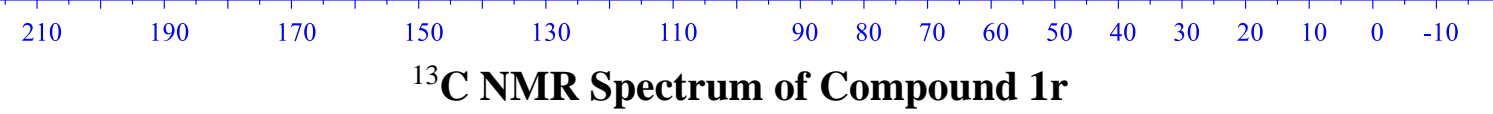



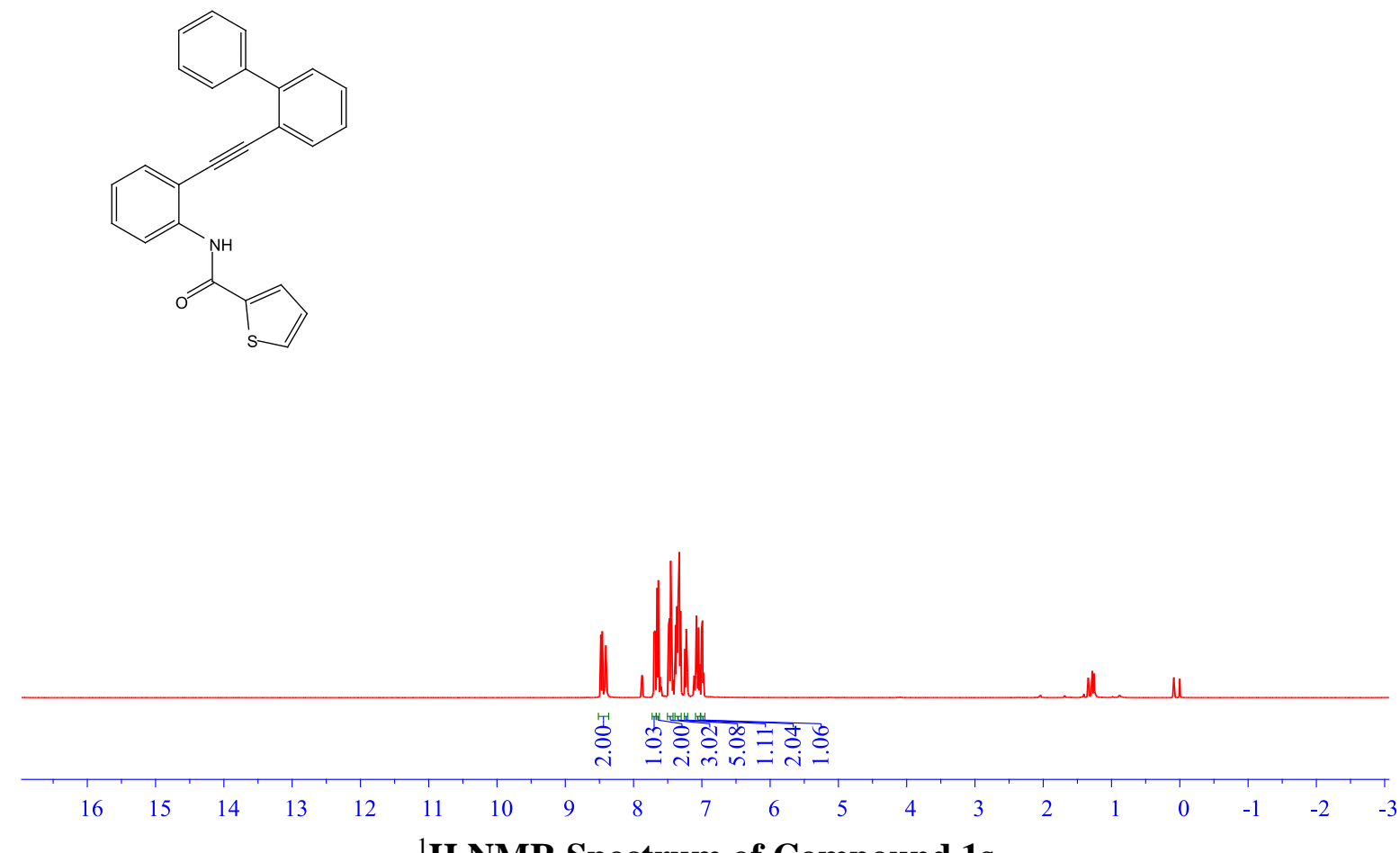

${ }^{1} \mathrm{H}$ NMR Spectrum of Compound $1 \mathrm{~s}$

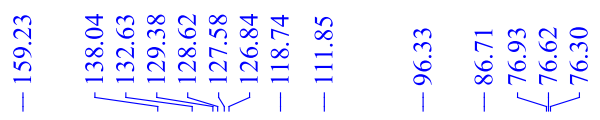
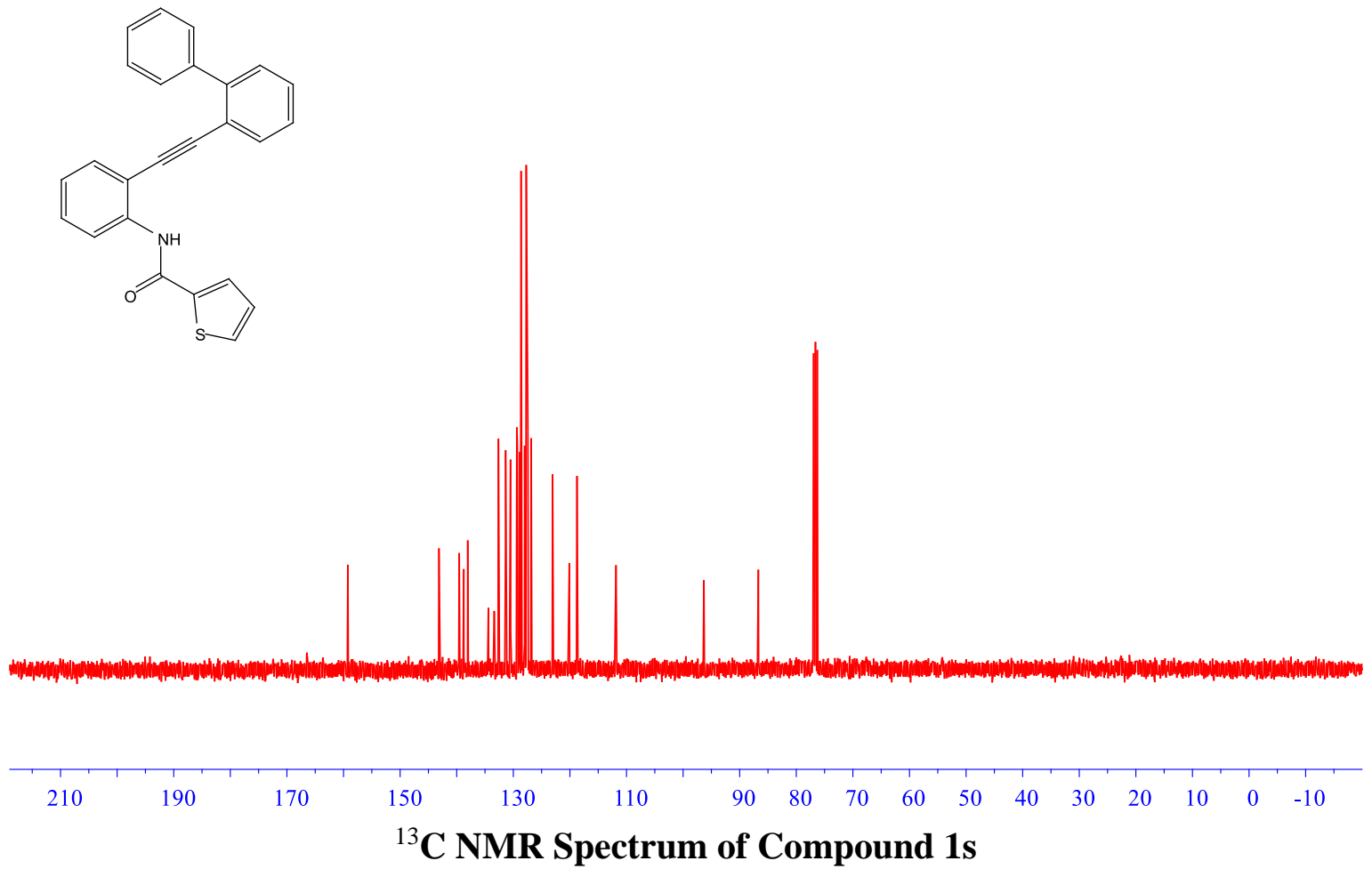


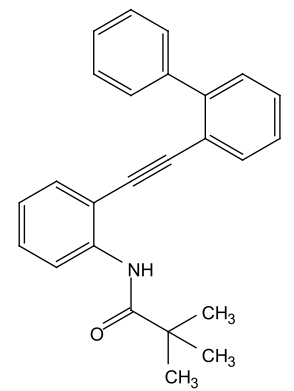

u N

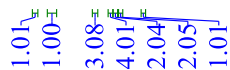

\section{$\stackrel{8}{\circ}$}

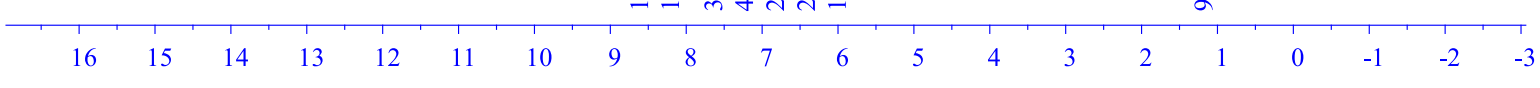

${ }^{1} \mathrm{H}$ NMR Spectrum of Compound 1t

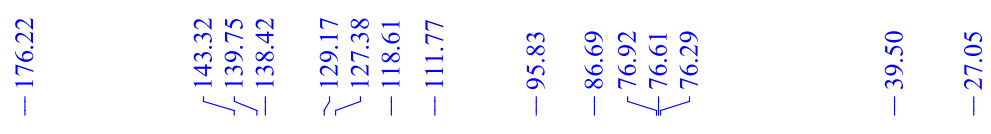<smiles>CC(C)(C)C(=O)Nc1ccccc1C#Cc1ccccc1-c1ccccc1</smiles>

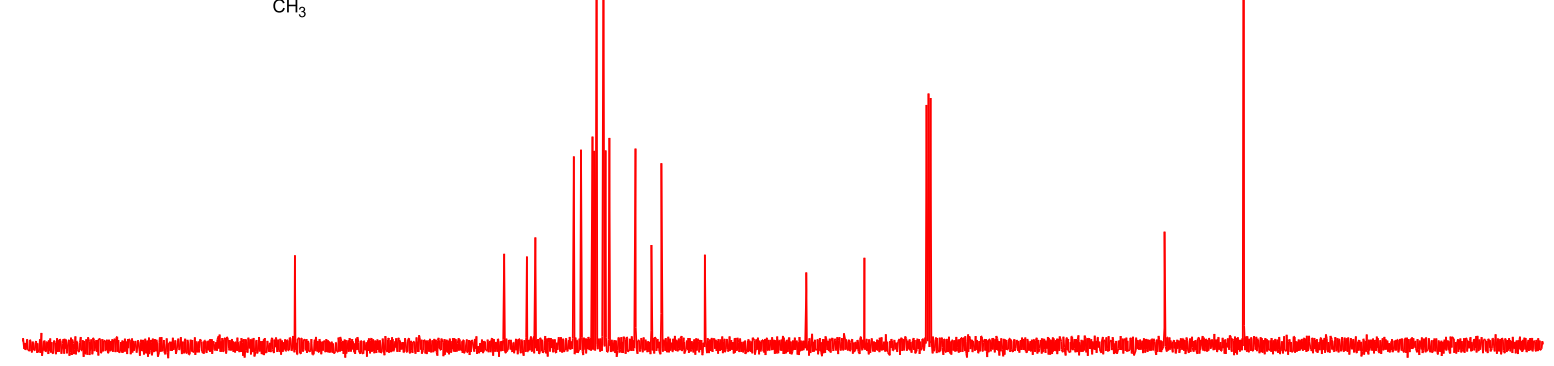

$\begin{array}{llllllllllllllllll}210 & 190 & 170 & 150 & 130 & 110 & 90 & 80 & 70 & 60 & 50 & 40 & 30 & 20 & 10 & 0 & -10\end{array}$

${ }^{13}$ C NMR Spectrum of Compound $1 \mathrm{t}$ 


\section{Kinetic isotope effect studies}

\section{Synthesis of compound 1a-D-1}
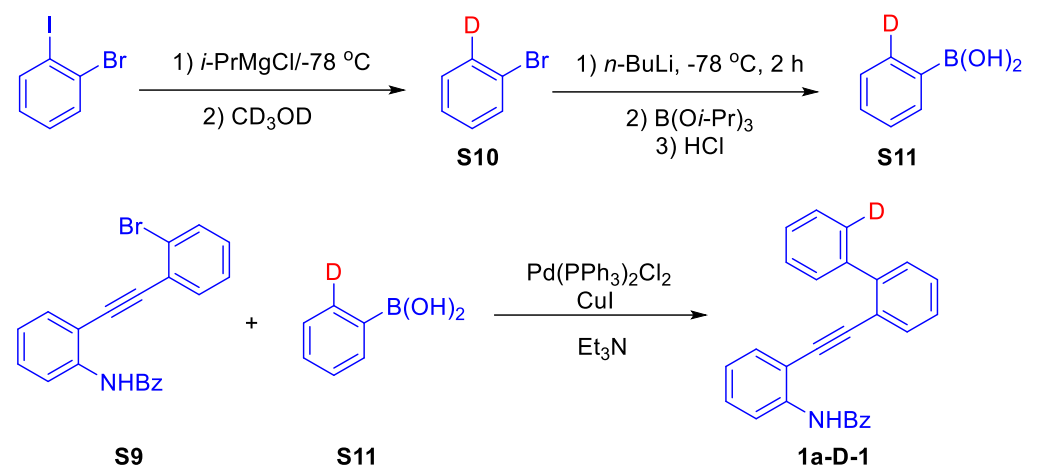

To a solution of 1-bromo-2-iodobenzene $(5 \mathrm{~g}, 17.7 \mathrm{mmol})$ in a mixture of THF and $\mathrm{Et}_{2} \mathrm{O}(120 \mathrm{~mL}$, 1:1) at $-78{ }^{\circ} \mathrm{C}$ was added dropwise isopropyl magnesium chloride $(2 \mathrm{M}$ in THF, $9.0 \mathrm{~mL}, 18.0$ $\mathrm{mmol}$ ). The mixture was stirred at that temperature for $2 \mathrm{~h}$ and then, $\mathrm{CD}_{3} \mathrm{OD}$ ( $\left.2 \mathrm{~g}, 55.5 \mathrm{mmol}\right)$ was added. The solution was slowly warmed to room temperature. $\mathrm{HCl}(10 \%$ aq., $100 \mathrm{~mL})$ was added and the resulting mixture was stirred for $30 \mathrm{~min}$. at room temperature. The aqueous layer was extracted with $\mathrm{Et}_{2} \mathrm{O}$ (3 x $30 \mathrm{~mL}$ ). The pure 2-deuterio bromobenzene $\mathbf{S 1 0}$ was obtained by distillation.

A two-neck $100 \mathrm{~mL}$ flask fitted with magnetic stirring bar, and low-temperature thermometer was charged with 2-deuterio bromobenzene S10 $(10.0 \mathrm{mmol})$ under nitrogen atmosphere. Dry THF $(25 \mathrm{~mL})$ was added, and the solution was cooled to $-78^{\circ} \mathrm{C}$. To this solution was added $n$-butyllithium $(6.25 \mathrm{~mL}, 1.6 \mathrm{M}, 10.0 \mathrm{mmol})$ drop wise using slow addition pump over 30 minutes. The solution was stirred at $-78^{\circ} \mathrm{C}$ for $2 \mathrm{~h}$ whereupon triisopropyl borate $(15.0 \mathrm{mmol})$ dissolved in $3 \mathrm{~mL}$ of dry THF was added drop wise to the reaction system. The solution was allowed to warm to room temperature overnight. After that the reaction was quenched with dilute $\mathrm{HCl}(20 \%, 20 \mathrm{~mL})$, and the reaction mixture was stirred for $3 \mathrm{~h}$ at room temperature. The resulted biphasic solution was extracted with $\mathrm{Et}_{2} \mathrm{O}(2 \times 15 \mathrm{~mL})$. The ethereal solution was washed twice with $\mathrm{H}_{2} \mathrm{O}$ and concentrated by rotary evaporation. To the crude product (viscous liquid), hexane $20 \mathrm{ml}$ was added. The white (phenyl-2-d)boronic acid (S11) solid precipitated in hexane was filtered and dried and used without further purification.

To an oven-dried three necked flask, S9 (2 mmol), (phenyl-2-d)boronic acid S11 (2.4 mmol), aqueous solution of $\mathrm{K}_{2} \mathrm{CO}_{3}(2 \mathrm{M}, 5 \mathrm{~mL})$ and DME $(4 \mathrm{~mL})$ were added under a gentle stream of nitrogen, and the mixture was stirred for $30 \mathrm{~min}$ at room temperature under $\mathrm{N}_{2}$ atmosphere. To the stirred mixture, $\mathrm{PdCl}_{2}\left(\mathrm{PPh}_{3}\right)_{2}(0.040 \mathrm{mmol})$ was added at room temperature, and the mixture was stirred for overnight at $80{ }^{\circ} \mathrm{C}$, under $\mathrm{N}_{2}$. The reaction mixture was then cooled to room temperature and diluted with EtOAc. The organic layer was washed with water and dried over $\mathrm{MgSO}_{4}$. After removing the volatiles in vacuo, the residue was subjected to column chromatography on silica gel (hexane/EtOAc $=30 / 1$ ) to afford 1a-D-1. White solid, ${ }^{1} \mathbf{H}$ NMR (400 MHz, CDCl 3 ) $\delta 8.62-8.50(\mathrm{~m}, 2 \mathrm{H}), 7.65(\mathrm{dd}, J=14.5,7.5 \mathrm{~Hz}, 3 \mathrm{H}), 7.61-7.57(\mathrm{~m}, 1 \mathrm{H})$, $7.51(\mathrm{t}, J=7.4 \mathrm{~Hz}, 1 \mathrm{H}), 7.44-7.33(\mathrm{~m}, 7 \mathrm{H}), 7.28-7.23(\mathrm{~m}, 2 \mathrm{H}), 7.21-7.16(\mathrm{~m}, 1 \mathrm{H}), 7.05(\mathrm{t}, J$ $=7.5 \mathrm{~Hz}, 1 \mathrm{H})$. 

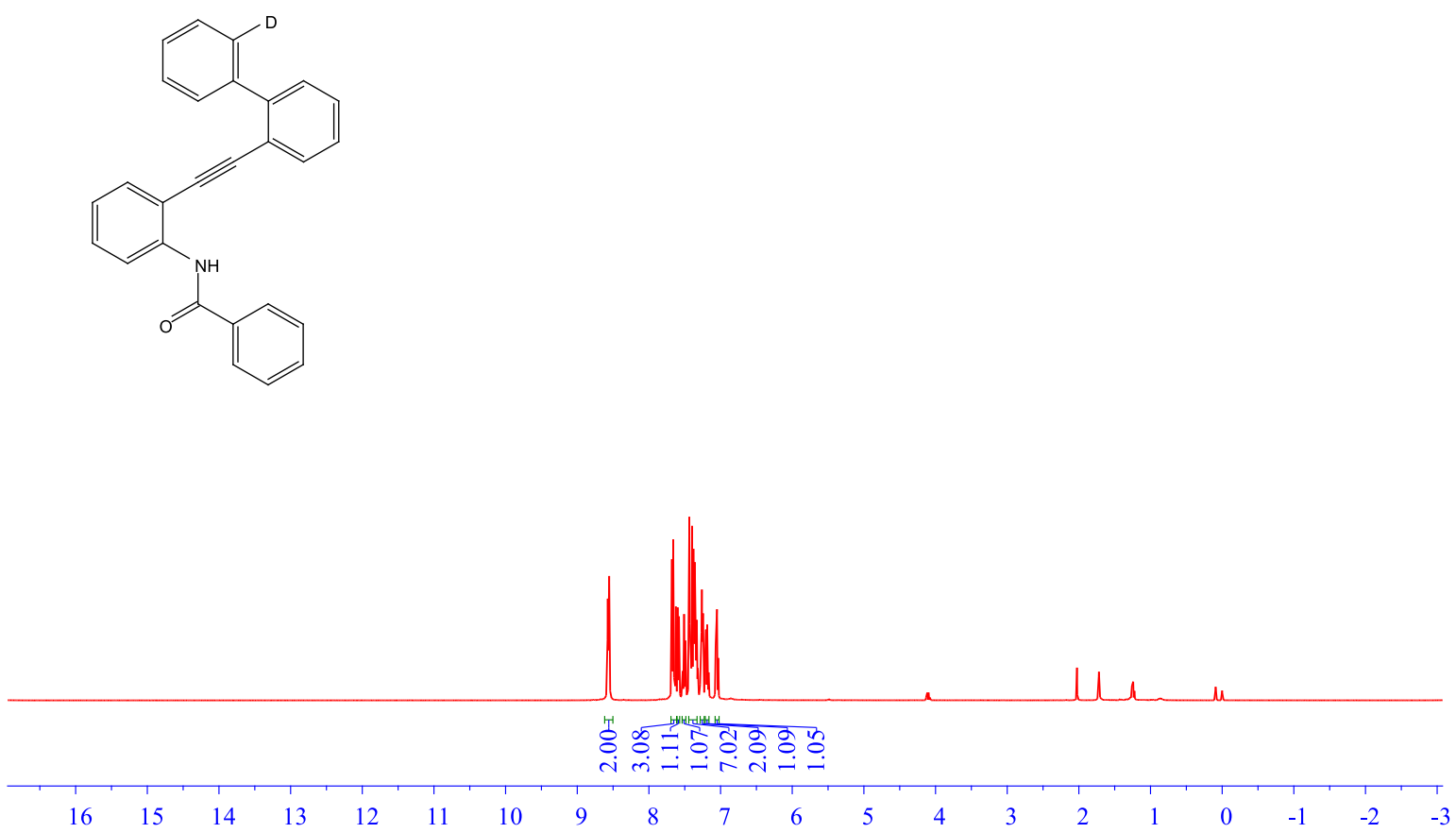

\section{Synthesis of compound 1a-D-5}

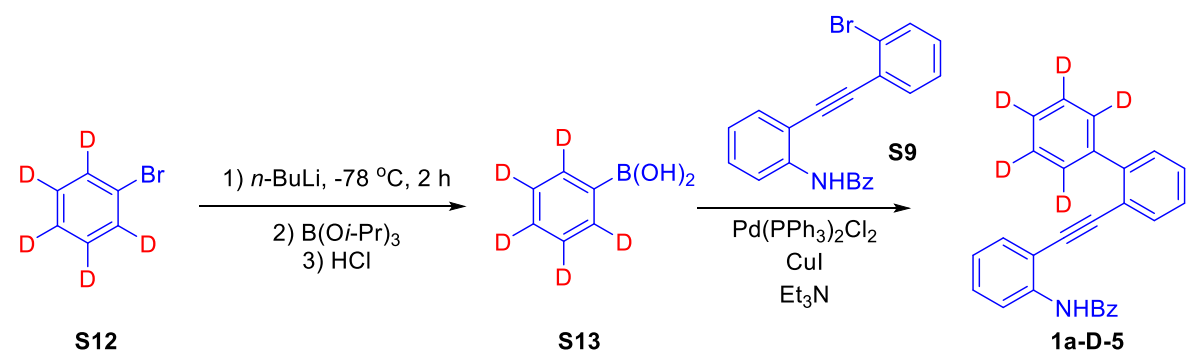

A two-neck $500 \mathrm{~mL}$ flask fitted with magnetic stirring bar, and low-temperature thermometer was charged with bromobenzene-D5 $(\mathbf{S 1 2}, 8.1 \mathrm{~mL}, 40.0 \mathrm{mmol})$ under nitrogen atmosphere. Dry THF $(100 \mathrm{~mL})$ was added, and the solution was cooled to $-78^{\circ} \mathrm{C}$. To this solution was added $n$-butyllithium $(25.0 \mathrm{~mL}, 1.6 \mathrm{M}, 40.0 \mathrm{mmol})$ drop wise using slow addition pump over 30 minutes. The solution was stirred at $-78^{\circ} \mathrm{C}$ for $2 \mathrm{~h}$ whereupon triisopropyl borate $(6.3 \mathrm{~g}, 60.0$ mmol) dissolved in $10 \mathrm{~mL}$ of dry THF was added drop wise to the reaction system. The solution was allowed to warm to room temperature overnight. After that the reaction was quenched with dilute $\mathrm{HCl}(20 \%, 70 \mathrm{~mL})$, and the reaction mixture was stirred for $3 \mathrm{~h}$ at room temperature. The resulted biphasic solution was extracted with Et2O (2 X $50 \mathrm{~mL})$. The ethereal solution was washed twice with $\mathrm{H} 2 \mathrm{O}$ and concentrated by rotary evaporation. To the crude product (viscous liquid), hexane $75 \mathrm{ml}$ was added. The white (d5-phenyl)boronic acid $\mathbf{S 1 3}$ solid precipitated in hexane was filtered and dried and used without further purification.

To an oven-dried three necked flask, S9 $(2 \mathrm{mmol})$, (d5-phenyl)boronic acid S13 (2.4 mmol), aqueous solution of $\mathrm{K}_{2} \mathrm{CO}_{3}(2 \mathrm{M}, 5 \mathrm{~mL})$ and DME $(4 \mathrm{~mL})$ were added under a gentle stream of nitrogen, and the mixture was stirred for $30 \mathrm{~min}$ at room temperature under $\mathrm{N}_{2}$ atmosphere. To the stirred mixture, $\mathrm{PdCl}_{2}\left(\mathrm{PPh}_{3}\right)_{2}(0.040 \mathrm{mmol})$ was added at room temperature, and the mixture 
was stirred for overnight at $80{ }^{\circ} \mathrm{C}$, under $\mathrm{N}_{2}$. The reaction mixture was then cooled to room temperature and diluted with EtOAc. The organic layer was washed with water and dried over $\mathrm{MgSO}_{4}$. After removing the volatiles in vacuo, the residue was subjected to column chromatography on silica gel (hexane/EtOAc $=30 / 1)$ to afford 1a-D-5. White solid, ${ }^{1} \mathbf{H}$ NMR $\left(400 \mathrm{MHz}, \mathbf{C D C l}_{3}\right) \delta 8.56(\mathrm{~d}, J=7.9 \mathrm{~Hz}, 2 \mathrm{H}), 7.66(\mathrm{dd}, J=11.8,7.9 \mathrm{~Hz}, 3 \mathrm{H}), 7.52(\mathrm{t}, J=7.4 \mathrm{~Hz}$, $1 \mathrm{H}), 7.45-7.33(\mathrm{~m}, 7 \mathrm{H}), 7.06(\mathrm{t}, J=7.6 \mathrm{~Hz}, 1 \mathrm{H})$.

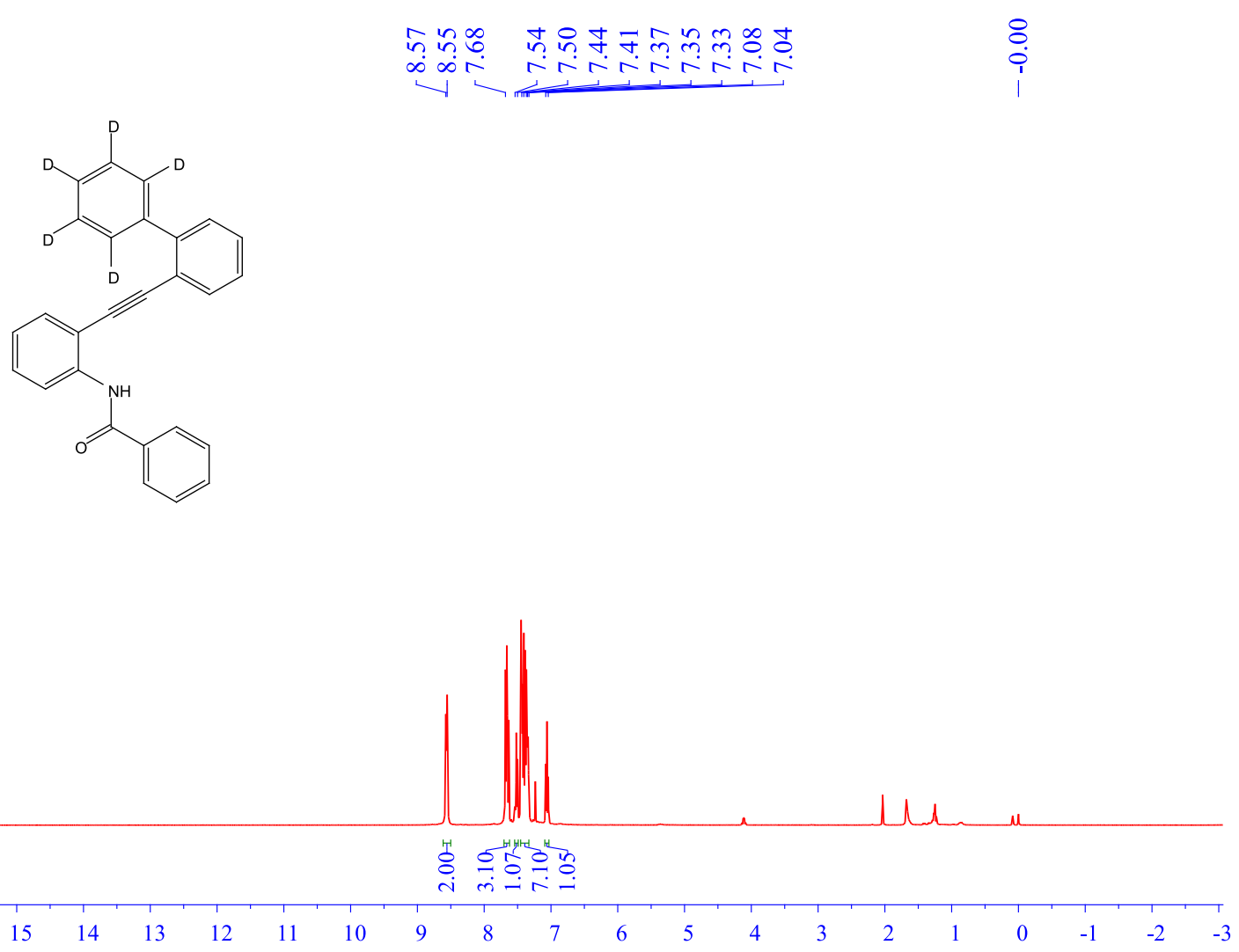

\section{Intramolecular Kinetic Isotope Effect of 1a-D-1:}

The substrate $N$-(2-(([1,1'-biphenyl]-2-yl-2'-d)ethynyl)phenyl)benzamide (1a-D-1, $0.5 \mathrm{mmol}$, $0.1866 \mathrm{~g})$, BQ (1.0 mmol, 0.1081g, 2.0 equiv.) and $\mathrm{Pd}(\mathrm{OAc})_{2}(0.05 \mathrm{mmol}, 0.0112 \mathrm{~g}, 10 \mathrm{~mol} \%)$ were added to a $25 \mathrm{~mL}$ Schlenk tube. The flask was evacuated and backfilled with $\mathrm{O}_{2}$, followed by addition of DMSO $(2.0 \mathrm{~mL})$. The mixture was stirred at $60{ }^{\circ} \mathrm{C}$ for $1 \mathrm{~h}$. The solution was then quenched by $\mathrm{H}_{2} \mathrm{O}$ and extracted with EtOAc, the combined organic layers were dried over $\mathrm{Na}_{2} \mathrm{SO}_{4}$, filtered, and evaporated under vaccum. The residue was purified by column chromatography on silica gel (eluent: light petroleum ether : ethyl acetate, $V: V=20: 1$ ) to afford the desired product product mixture $\mathbf{2 a}+\mathbf{2 a - D - 1}$. The intramolecular kinetic isotopic effect $\mathrm{K}_{\mathrm{H}} / \mathrm{K}_{\mathrm{D}}=1.94$ was determined by ${ }^{\mathbf{1}} \mathbf{H} \mathbf{~ N M R}$. 


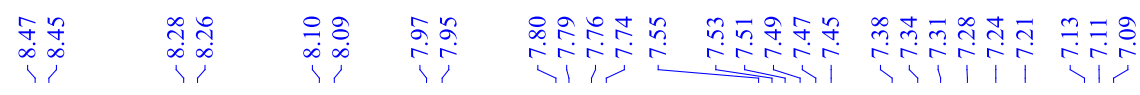

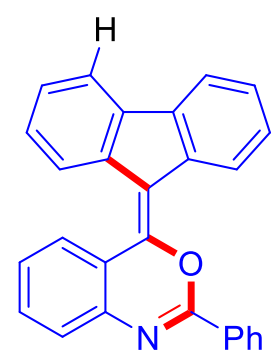
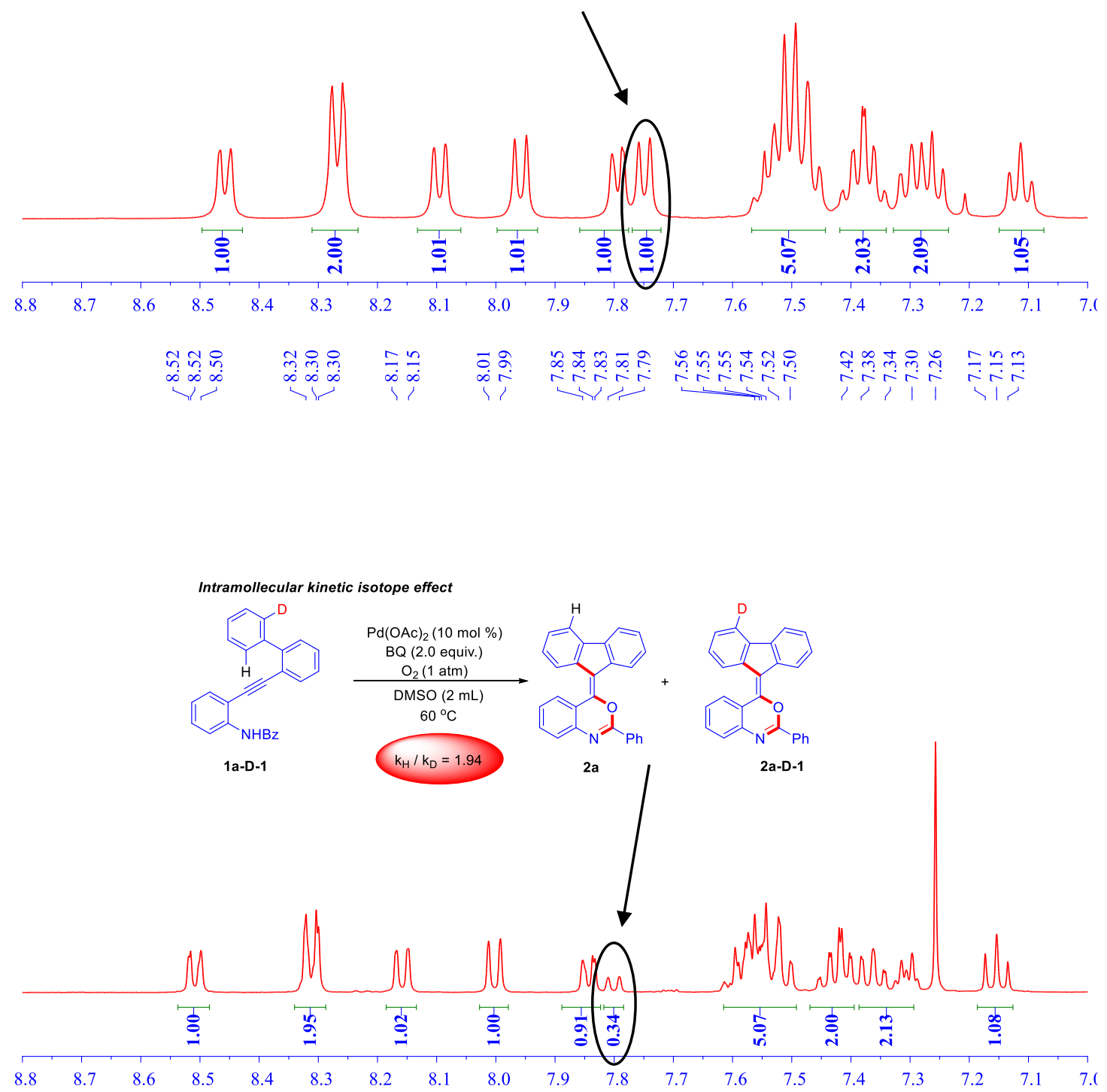
The Kinetic Isotope Effect of Intermolecular Experiments Between 1a and 1a-D-5:

To two $25 \mathrm{~mL}$ Schlenk tubes, were added BQ (0.5 mmol, 0.0504g, 2.0 equiv.) and $\mathrm{Pd}(\mathrm{OAc})_{2}$ $(0.025 \mathrm{mmol}, 0.0056 \mathrm{~g}, 10 \mathrm{~mol} \%)$, separately. One tube was added 1a $(0.0933 \mathrm{~g}, 0.25 \mathrm{mmol})+$ DMSO (2.0 mL) and another one was added 1a-D-5 (0.0946 g, $0.25 \mathrm{mmol})+$ DMSO (2.0 mL). Then they were evacuated and backfilled with $\mathrm{O}_{2}$ and stirred at $60^{\circ} \mathrm{C}$ for $1 \mathrm{~h}$. At the end of the reaction, the mixtures of these two tubes were combined, the solution was then quenched by $\mathrm{H}_{2} \mathrm{O}$ and extracted with EtOAc, the combined organic layers were dried over $\mathrm{Na}_{2} \mathrm{SO}_{4}$, filtered, and evaporated under vaccum. The residue was purified by column chromatography on silica gel (eluent: light petroleum ether : ethyl acetate, $V: V=20: 1$ ) to afford the desired product product mixture 2a + 2a-D-4. The intermolecular kinetic isotopic effect $\mathrm{K}_{\mathrm{H}} / \mathrm{K}_{\mathrm{D}}=2.57$ was determined by ${ }^{1} \mathrm{H}$ NMR. 

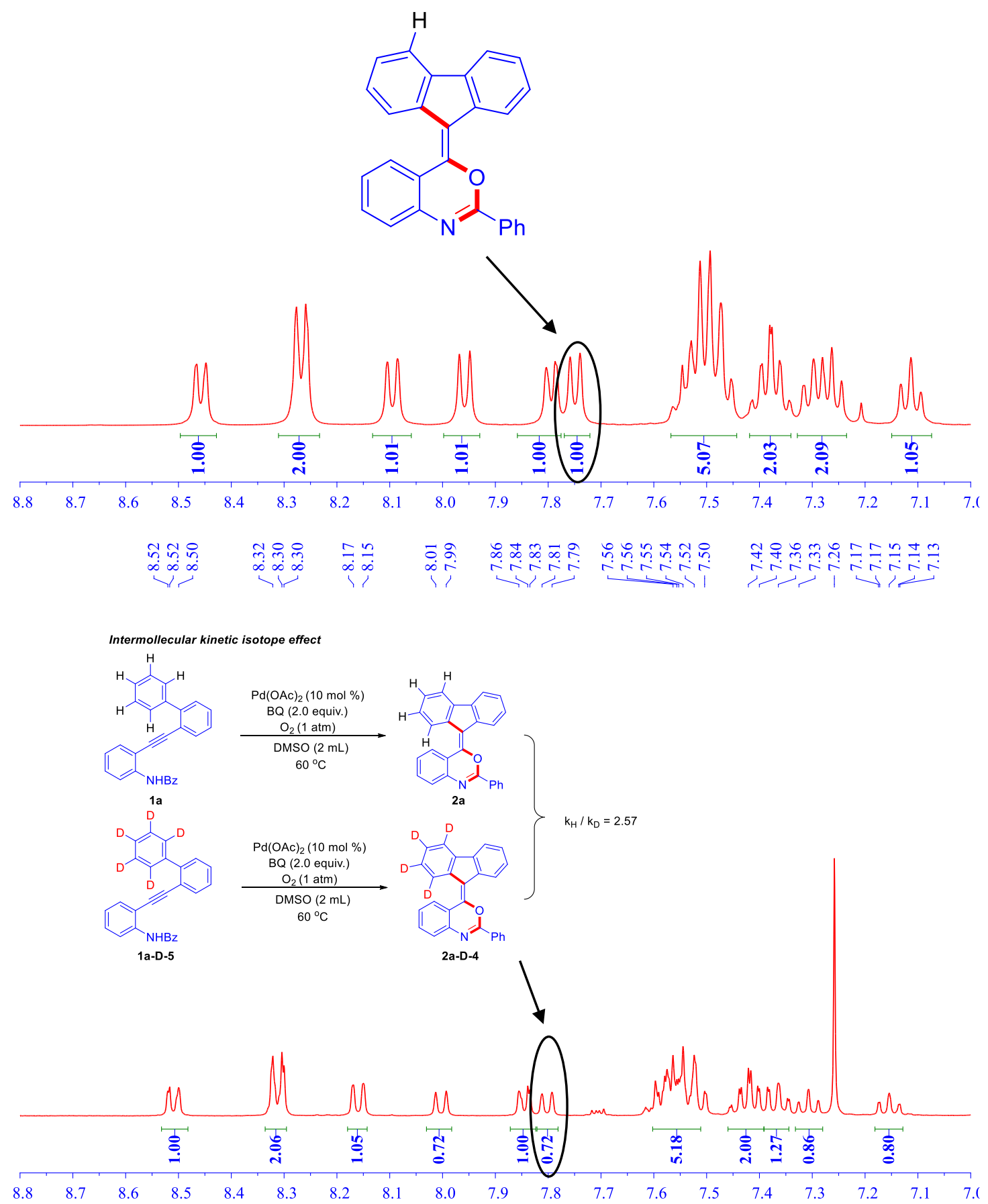